\title{
MIRABOLIC SATAKE EQUIVALENCE AND SUPERGROUPS
}

\author{
ALEXANDER BRAVERMAN, MICHAEL FINKELBERG, VICTOR GINZBURG, \\ AND ROMAN TRAVKIN \\ To our friend Sasha Shen on the occasion of his 60th birthday
}

\begin{abstract}
We construct a mirabolic analogue of the geometric Satake equivalence. We also prove an equivalence that relates representations of a supergroup with the category of $\mathrm{GL}(N-1, \mathbb{C} \llbracket t \rrbracket)$-equivariant perverse sheaves on the affine Grassmannian of $\mathrm{GL}_{N}$. We explain how our equivalences fit into a more general framework of conjectures due to Gaiotto and to Ben-Zvi, Sakellaridis and Venkatesh.
\end{abstract}

\section{Contents}

1. Introduction 2

1.1. Reminder on Geometric Satake 2

1.2. Mirabolic Satake category 3

1.3. Mirabolic Satake equivalence 4

1.4. Conjectural Iwahori-equivariant version 6

1.5. Satake equivalence for some Lie supergroups 6

1.6. Gaiotto conjectures 7

1.7. Conjectures of Ben-Zvi, Sakellaridis and Venkatesh 8

1.8. Organization of the paper 9

1.9. Acknowledgments 9

2. Gaiotto conjectures 9

2.1. Reminder on strong actions on categories 9

2.2. Digression on the local (quantum) geometric Langlands
correspondence

2.3. Whittaker category and the fundamental local equivalence (FLE) 11

2.4. Gaiotto conjectures: geometric Langlands form in the case $N>M \quad 13$

2.5. Gaiotto conjectures: geometric Langlands form for $N=M \quad 15$

2.6. Gaiotto conjectures: "simple-minded" form 15

3. A coherent realization of $D_{\mathrm{GL}(N, \mathbf{O})}(\mathbf{G r} \times \mathbf{V}) \quad 16$

3.1. Setup and notation 16

3.2. Constructible mirabolic category and convolutions 17

Mathematics Subject Classification (2020). 14F08, (14D24, 14M15, 17B20).

Key words. Satake equivalence, Mirabolic affine Grassmannian, Supergroups. 
3.3. Fusion 19

3.4. Coherent mirabolic category and convolutions 19

3.5. Localization, coherent 21

3.6. Renormalizations 21

3.7. Super 23

3.8. Koszul equivalence 23

3.9. Deequivariantized Ext algebra 24

3.10. Localization, constructible 26

3.11. Calculation of the Ext algebra 28

3.12. Restriction to $\mathbf{G r} \times \mathbf{V}_{0} \quad 29$

3.13. Some invariant theory 34

3.14. The monoidal property of $\Phi^{2,0} \quad 35$

3.15. Fourier Transform 36

3.16. The monoidal property of $\Phi^{1,1} \quad 37$

4. A coherent realization of $D_{\mathrm{GL}(N-1, \mathbf{O})}(\mathbf{G r}) \quad 38$

4.1. Notation 38

4.2. Constructible mirabolic restriction 39

4.3. Coherent mirabolic restriction 40

4.4. Proof of Theorem 4.1.1 43

5. Loop rotation and quantization 44

5.1. Graded differential operators and convolutions 44

5.2. Construction of equivalences 45

5.3. Restriction to $\mathbf{G r} \times \mathbf{V}_{0}$ with loop rotation 46

5.4. $\mathfrak{D}$-modules and $D_{\mathrm{GL}(N-1, \mathbf{O}) \rtimes \mathbb{C}^{\times}}(\mathbf{G r}) \quad 47$

References $\quad 47$

\section{Introduction}

1.1. Reminder on Geometric Satake. Let $\mathbf{F}=\mathbb{C}((t)) \supset \mathbb{C} \llbracket t \rrbracket=\mathbf{O}$. Throughout the paper, we fix an integer $N \geq 1$, set $\mathbf{G}_{\mathbf{F}}=\mathrm{GL}(N, \mathbf{F})$, resp. $\mathbf{G}_{\mathbf{O}}=$ $\operatorname{GL}(N, \mathbf{O})$, and let $\mathbf{G r}=\mathbf{G}_{\mathbf{F}} / \mathbf{G}_{\mathbf{O}}$ be the affine Grassmannian of $\mathrm{GL}_{N}$. This is an ind-scheme equipped with a natural action of the group $\mathbf{G}_{\mathbf{O}} \rtimes \mathbb{C}^{\times}$, where $\mathbb{C}^{\times}$acts by loop rotation. Let $D_{\mathbf{G}_{\mathbf{O}}}(\mathbf{G r})$, resp. $D_{\mathbf{G}_{\mathbf{O}} \rtimes \mathbb{C}^{\times}}(\mathbf{G r})$, be the $\mathbf{G}_{\mathbf{O}^{-}}$equivariant, resp. $\mathbf{G}_{\mathbf{O}} \rtimes \mathbb{C}^{\times}$-equivariant, constructible derived category of $\mathbf{G r} .{ }^{1}$ This is a monoidal category with respect to convolution (that coincides with fusion), cf. [MV].

Let $\mathfrak{g l}_{N}$ be the complex vector space of $N \times N$-matrices and let $\mathrm{GL}_{N}$ act on $\mathfrak{g l}_{N}$ by conjugation. Write $\operatorname{Sym}^{\bullet}\left(\mathfrak{g l}_{N}[-2]\right)$ for the symmetric algebra of $\mathfrak{g l}_{N}$ viewed as a dg-algebra such that the space $\mathfrak{g l}_{N}$, of generators, is placed in degree 2 and

\footnotetext{
${ }^{1}$ Throughout the paper, we consider the sheaves with complex coefficients (with a few technical exceptions).
} 
the differential is equal to zero. Let $D_{\text {perf }}^{\mathrm{GL}_{N}}\left(\operatorname{Sym}^{\bullet}\left(\mathfrak{g l}_{N}[-2]\right)\right)$ be the triangulated category of perfect $\mathrm{GL}_{N}$-equivariant dg-modules over $\operatorname{Sym}^{\bullet}\left(\mathfrak{g l}_{N}[-2]\right)$, localized with respect to quasiisomorphisms. Tensor product of dg-modules gives this category a monoidal structure. One of the versions of derived Satake equivalence proved in $[\mathrm{BF}]$ states that there is an equivalence $D_{\text {perf }}^{\mathrm{GL}_{N}}\left(\operatorname{Sym}^{\bullet}\left(\mathfrak{g l}_{N}[-2]\right)\right) \stackrel{\sim}{\longrightarrow}$ $D_{\mathbf{G}_{\mathbf{O}}}(\mathbf{G r})$, of triangulated monoidal categories.

It will be convenient for us to reformulate the above result as follows. Let $T^{*} \mathrm{GL}_{N}[2]$ be the shifted cotangent bundle on $\mathrm{GL}_{N}$, viewed as a dg-scheme equipped with zero differential. The action of $\mathrm{GL}_{N}$ on itself by left and right translations induces a $\mathrm{GL}_{N} \times \mathrm{GL}_{N}$-action on $T^{*} \mathrm{GL}_{N}[2]$. Let $D_{\text {perf }}^{\mathrm{GL}_{N} \times \mathrm{GL}_{N}}\left(T^{*} \mathrm{GL}_{N}[2]\right)$ be the triangulated category of $\mathrm{GL}_{N} \times \mathrm{GL}_{N}$-equivariant perfect complexes of

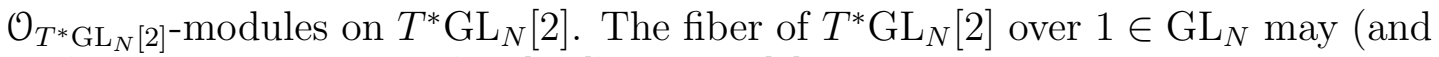
will) be identified with $\left(\mathfrak{g l}_{N}[-2]\right)^{*}=\mathfrak{g l}_{N}^{*}[2]$. Restriction to this fiber induces a monoidal equivalence $D_{\text {perf }}^{\mathrm{GL}_{N} \times \mathrm{GL}_{N}}\left(T^{*} \mathrm{GL}_{N}[2]\right) \stackrel{\sim}{\longrightarrow} D_{\text {perf }}^{\mathrm{GL}_{N}}\left(\mathrm{Sym}^{\bullet}\left(\mathfrak{g l}_{N}[-2]\right)\right)$, where the category on the right is identified with the triangulated category of $\mathrm{GL}_{N}$-equivariant perfect complexes on $\left(\mathfrak{g l}_{N}[-2]\right)^{*}$. Thus, the derived Satake equivalence stated above may be interpreted as an equivalence $D_{\text {perf }}^{\mathrm{GL}_{N} \times \mathrm{GL}_{N}}\left(T^{*} \mathrm{GL}_{N}[2]\right) \stackrel{\sim}{\longrightarrow} D_{\mathrm{G}_{\mathbf{O}}}(\mathbf{G r})$.

There is also a natural 'quantum' counterpart of the latter equivalence, where the category $D_{\text {perf }}^{\mathrm{GL}_{N} \times \mathrm{GL}_{N}}\left(T^{*} \mathrm{GL}_{N}[2]\right)$ is replaced by an appropriately defined category of asymptotic shifted weakly equivariant $D$-modules on $\mathrm{GL}_{N}$, and the category $D_{\mathbf{G}_{\mathbf{O}}}(\mathbf{G r})$ is replaced by $D_{\mathbf{G}_{\mathbf{O}} \rtimes \mathbb{C} \times}(\mathbf{G r})$, see $[\mathrm{BF}]$.

1.2. Mirabolic Satake category. In the present paper we will be interested in a mirabolic analogue of the above setting. To explain this, fix an $N$-dimensional vector space $V$ and put $\mathbf{V}=V \otimes \mathbf{F}$, resp. $\mathbf{V}_{0}=V \otimes \mathbf{O}$ and $\stackrel{\circ}{\mathbf{V}}=\mathbf{V} \backslash\{0\}$. We identify $\mathbf{G}=\mathrm{GL}_{N}$ with $\mathrm{GL}(V)$, so that $\mathbf{G}_{\mathbf{F}}$ acts on $\mathbf{V}$ and $\mathbf{G}_{\mathbf{O}}$ is the stabilizer of $\mathbf{V}_{0}$. Following [FGT], the mirabolic affine Grassmannian is defined as $\mathbf{G r} \times \mathbf{V}$. We let $\mathbf{G}_{\boldsymbol{O}}$ act on $\mathbf{G r} \times \mathbf{V}$ diagonally. The orbits of $\mathbf{G}_{\mathbf{O}}$ in $\mathbf{G r} \times \stackrel{\circ}{\mathbf{V}}$ are, unlike the case of $\mathbf{G}_{\mathbf{O}}$-orbits in $\mathbf{G r}$, not finite dimensional. Heuristically, these orbits are of semi-infinite type in the sense that the 'closure' of an orbit projects onto a (finite dimensional) Schubert variety in $\mathbf{G r}$ and onto a lattice in $\mathbf{V}$.

In view of the above, defining the correct mirabolic anague of the equivariant derived Satake category requires some care. According to our definition, an object of this category is supported on the product of a finite-dimensional Schubert variety in $\mathbf{G r}$ and a lattice inside the Tate vector space $\mathbf{V}$; moreover, this object is pulled back from a finite-dimensional quotient of this lattice. According to three possible choices (!-,*-, or !*-) of pull-back, one gets the three versions $D_{! \mathbf{G}_{\mathbf{O}}}(\mathbf{G r} \times \mathbf{V}), D_{* \mathbf{G}_{\mathbf{O}}}(\mathbf{G r} \times \mathbf{V}), D_{! * \mathbf{G}_{\mathbf{O}}}(\mathbf{G r} \times \mathbf{V})$ of $\mathbf{G}_{\mathbf{O}}$-equivariant constructible derived categories on $\mathbf{G r} \times \mathbf{V}$. These categories are related to each other by certain renormalization equivalences. 
We equip the above categories with monoidal structures given by various types of convolution operation. The convolution along the Grassmannian, the first factor in $\mathbf{G r} \times \mathbf{V}$, is defined similarly to the case of the usual Satake category. The convolution along the second factor depends on the choice of category. Specifically, the convolution operation $\stackrel{!}{\circledast}$ in $D_{!_{\mathbf{G}_{\mathbf{O}}}}(\mathbf{G r} \times \mathbf{V})$ involves the !-tensor product of constructible sheaves on $\mathbf{V}$. The convolution operation ${ }^{*}$ on $D_{* \mathbf{G}_{\mathbf{O}}}(\mathbf{G r} \times \mathbf{V})$ involves a $*$-push-forward along $+: \mathbf{V} \times \mathbf{V} \rightarrow \mathbf{V}$, the map given by addition. These two types of convolution are related to each other via Fourier Transform (along V). Finally, we define a monoidal structure $\star$ on $D_{! * \mathbf{G}_{\mathbf{O}}}(\mathbf{G r} \times \mathbf{V})$ via the fusion operation on a mirabolic analogue of the Beilinson-Drinfeld Grassmannian.

The categories above have natural counterparts involving the action of $\mathbb{C}^{\times}$on Gr by loop rotation. These are $\mathbb{C}[\hbar]$-linear categories where $\mathbb{C}[\hbar]=H_{\mathbb{C}^{\times}}(\mathrm{pt})$. Thus, there is a category $D_{! \mathbf{G}_{\mathbf{O}} \rtimes \mathbb{C}^{\times}}(\mathbf{G r} \times \mathbf{V})$, resp. $D_{* \mathbf{G}_{\mathbf{O}} \rtimes \mathbb{C} \times}(\mathbf{G r} \times \mathbf{V})$, equipped with a similarly defined monoidal structure $\stackrel{!}{\circledast}$, resp. $*$ * The fusion operation $\star$ on $D_{! * \mathbf{G}_{\mathbf{O}}}(\mathbf{G r} \times \mathbf{V})$ has $n o \mathbf{G}_{\mathbf{O}} \rtimes \mathbb{C}^{\times}$-equivariant counterpart, however.

1.3. Mirabolic Satake equivalence. The category on the 'other side' of mirabolic Satake equivalence is an appropriately defined triangulated category of "equivariant asymptotic shifted $D$-modules" on the vector space $\mathfrak{g l}_{N}$ of $N \times N$-matrices. More formally, we introduce a $\mathbb{C}[\hbar]$-algebra $\mathfrak{D}^{\bullet}$, a graded version of the algebra of differential operators on $\mathfrak{g l}_{N}$ (where $\operatorname{deg} \hbar=2$ ). The action of $\mathrm{GL}_{N}$ on $\mathfrak{g l}_{N}$ by left and right multiplication induces a $\mathrm{GL}_{N} \times \mathrm{GL}_{N}$-action on $\mathfrak{D}^{\bullet}$. The relevant category is then defined to be the derived category of weakly $\mathrm{GL}_{N} \times \mathrm{GL}_{N}$-equivariant perfect dg-modules over $\mathfrak{D}^{\bullet}$, where $\mathfrak{D}^{\bullet}$ is viewed as a dg-algebra with zero differential. Similarly to the constructible story, we actually define three versions $D_{\text {perf }}^{\mathrm{GL}_{N} \times \mathrm{GL}_{N}}\left(\mathfrak{D}_{1,1}^{\bullet}\right)$, resp. $D_{\text {perf }}^{\mathrm{GL}_{N} \times \mathrm{GL}_{N}}\left(\mathfrak{D}_{0,2}^{\bullet}\right)$ and $D_{\text {perf }}^{\mathrm{GL}_{N} \times \mathrm{GL}_{N}}\left(\mathfrak{D}_{2,0}^{\bullet}\right)$, of such a category that correspond to three different choices of grading on our algebra. The algebra of asymptotic differential operators specializes at $\hbar=0$ to the algebra $\mathbb{C}\left[T^{*} \mathfrak{g l}_{N}\right]$. We denote the specialization of $\mathfrak{D}_{i, 2-i}^{\bullet}$ at $\hbar=0$ by $\mathfrak{S}_{i, 2-i}^{\bullet}$. Accordingly, the above defined $\mathbb{C}[\hbar]$-linear categories specialize at $\hbar=0$ to various versions of the derived category of $\mathrm{GL}_{N} \times \mathrm{GL}_{N}$-equivariant coherent sheaves on shifted cotangent bundle $T^{*} \mathfrak{g l}_{N}$.

Next, we equip the above defined categories with monoidal structures. The monoidal structure $\stackrel{A}{*}$ on $D_{\text {perf }}^{\mathrm{GL}_{N} \times \mathrm{GL}_{N}}\left(\mathfrak{D}_{2,0}^{\bullet}\right)$ is defined as a convolution operation on $D$-modules associated with the map $\mathfrak{g l}_{N} \times \mathfrak{g l}_{N} \rightarrow \mathfrak{g l}_{N}$, given by matrix multiplication. The monoidal structure $\stackrel{B}{*}$ on $D_{\text {perf }}^{\mathrm{GL}_{N} \times \mathrm{GL}_{N}}\left(\mathfrak{D}_{0,2}^{\bullet}\right)$ is defined as $\mathrm{F}^{-1} \circ(*) \circ(\mathrm{F} \times \mathrm{F})$, where $\mathrm{F}$ is the functor of Fourier Transform on $D$-modules. Each of these monoidal structures has a quasiclassical limit at $\hbar=0$, defined 
as a convolution of coherent sheaves arising from a certain Lagrangian correspondence. Finally, tensor product of coherent sheaves, that is, the functor $\mathcal{M}, \mathcal{M}^{\prime} \mapsto \mathcal{M} \otimes_{\mathfrak{S}_{i, 1}} \mathcal{M}^{\prime}$, gives a monoidal structure on $D_{\text {perf }}^{\mathrm{GL}_{N} \times \mathrm{GL}_{N}}\left(\mathfrak{S}_{1,1}^{\bullet}\right)$. This monoidal structure has no counterpart for $D$-modules, i.e. for $\hbar \neq 0$.

One of the main results of the present paper, see Theorems 3.6.1,5.1.1, states that one has the following equivalences of triangulated monoidal categories, called mirabolic Satake equivalences:

$$
\begin{aligned}
& \left(D_{\text {perf }}^{\mathrm{GL}_{N} \times \mathrm{GL}_{N}}\left(\mathfrak{D}_{2,0}^{\bullet}\right), \quad \begin{array}{c}
* \\
*
\end{array}\right) \stackrel{\Phi_{\hbar}^{2,0}}{\cong}\left(D_{! \mathrm{G}_{\mathbf{O}} \times \mathbb{C}^{\times}}(\mathbf{G r} \times \mathbf{V}), \stackrel{!}{\circledast}\right) ;
\end{aligned}
$$

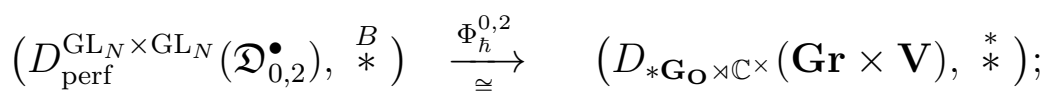

$$
\begin{aligned}
& \left(D_{\text {perf }}^{\mathrm{GL}_{N} \times \mathrm{GL}_{N}}\left(\mathfrak{S}_{1,1}^{\bullet}\right), \otimes_{\mathfrak{S}_{\mathbf{1}, 1}}\right) \stackrel{\Phi^{1,1}}{\cong} \quad\left(D_{! * \mathbf{G}_{\mathbf{O}}}(\mathbf{G r} \times \mathbf{V}), \star\right) .
\end{aligned}
$$

Furthermore, it turns out that the triangulated category $D_{! * \mathbf{G}_{\mathbf{O}}}(\mathbf{G r} \times \mathbf{V})$ is equivalent to the bounded derived category of the abelian category $\operatorname{Perv}_{\mathbf{G}_{\mathbf{O}}}(\mathbf{G r} \times \mathbf{V})$ of $\mathbf{G}_{\mathbf{O}^{-}}$equivariant perverse sheaves on $\mathbf{G r} \times \mathbf{V}$, the heart of the perverse t-structure on $D_{! * \mathbf{G}_{\mathbf{O}}}(\mathbf{G r} \times \mathbf{V})$.

Remark 1.3.1. The counterpart of the last statement in the case of the usual Satake category is false: the triangulated category $D_{! * \mathbf{G}_{\mathbf{O}}}(\mathbf{G r})$ is not equivalent to the derived category of the category $\operatorname{Perv}_{\mathbf{G}_{\mathbf{O}}}(\mathbf{G r})$, which is well known to be a semisimple abelian category.

Remark 1.3.2. One can view the group $\mathrm{GL}_{N}$ as a Zariski open subset of the vector space $\mathfrak{g l}_{N}$, of all $N \times N$-matrices. Associated with the open imbedding $\mathrm{GL}_{N} \hookrightarrow \mathfrak{g l}_{N}$, one has a restriction functor on $D$-modules. It turns out that the counterpart of this functor for constructible derived categories is a suitably defined version of restriction with respect to the 'zero section' $\mathbf{G r} \times\{0\} \hookrightarrow \mathbf{G r} \times \mathbf{V}$. That is, one has a natural functor $D_{\mathbf{G}_{\mathbf{O}}}(\mathbf{G r} \times \mathbf{V}) \rightarrow D_{\mathbf{G}_{\mathbf{O}}}(\mathbf{G r})$. We will show that the later functor makes the derived Satake category $D_{\mathbf{G}_{\mathbf{O}}}(\mathbf{G r})$ a localization of the mirabolic derived Satake category $D_{\mathbf{G}_{\mathbf{O}}}(\mathbf{G r} \times \mathbf{V})$. Moreover, the mirabolic Satake equivalence is compatible with the standard Satake equivalence in the sense that there is a commutative diagram of functors

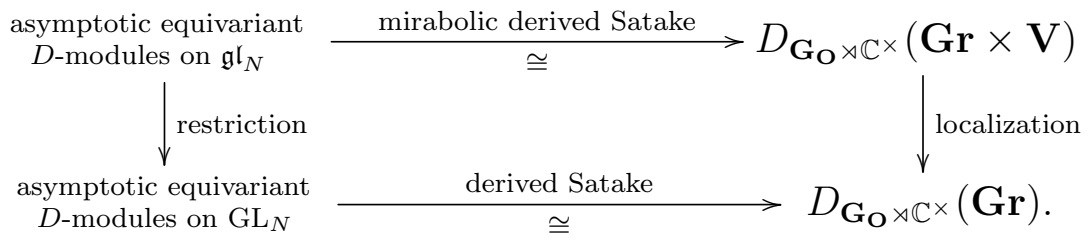


1.4. Conjectural Iwahori-equivariant version. Let $\mathbf{I} \subset \mathbf{G}_{\mathbf{O}}$ be an Iwahori subgroup and $\mathbf{F l}:=\mathbf{G}_{\mathbf{F}} / \mathbf{I}$ the affine flag variety. In [B], Bezrukavnikov established an equivalence of $D_{\mathbf{I}}(\mathbf{F l})$, the $\mathbf{I}$-equivariant constructible derived category of $\mathbf{F l}$, and the derived category of $\mathrm{GL}_{N^{-}}$equivariant coherent sheaves on an appropriate dg-version of the Steinberg variety. Motivated by this result, we expect that there is a mirabolic counterpart of this equivalence.

To explain this, fix a pair $V_{1}, V_{2}$, of $N$-dimensional vector spaces and let $\mathcal{F} \ell_{i}, i=$ 1,2 , denote the variety of complete flags in $V_{i}$. Further, consider a dg-scheme with zero differential

$$
\mathrm{H}_{\text {mir }}:=\operatorname{Hom}\left(V_{1}, V_{2}\right)[1] \times \operatorname{Hom}\left(V_{2}, V_{1}\right)[1] \times \mathcal{F} \ell_{1} \times \mathcal{F} \ell_{2} \cdot{ }^{2}
$$

Write $A$, resp. $B$, for an element of $\operatorname{Hom}\left(V_{1}, V_{2}\right)[1], \operatorname{resp} \operatorname{Hom}\left(V_{2}, V_{1}\right)[1]$, and $F_{i}=\left(F_{i}^{(1)} \subset F_{i}^{(2)} \subset \ldots \subset F_{i}^{(N)}=V_{i}\right)$ for an element of $\mathcal{F} \ell_{i}$.

We define the mirabolic Steinberg scheme to be a dg-subscheme $\mathrm{St}_{\text {mir }}$ of $\mathrm{H}_{\text {mir }}$ cut out by the equations saying that the flag $F_{2}$ is stable under the composition $A B$ and the flag $F_{1}$ is stable under the composition $B A$. Thus the mirabolic Steinberg scheme is a shifted variety of quadruples:

$\mathrm{St}_{\text {mir }}=\left\{\left(A, B, F_{1}, F_{2}\right) \in \mathrm{H}_{\text {mir }} \mid A B\left(F_{2}^{(j)}\right) \subseteq F_{2}^{(j)} \& B A\left(F_{1}^{(j)}\right) \subseteq F_{1}^{(j)}, \forall j \in[1, N]\right\}$.

Let $D_{\mathbf{I}}(\mathbf{F l} \times \mathbf{V})$ be the $\mathbf{I}$-equivariant constructible derived category of $\mathbf{F l} \times \mathbf{V}$. We propose the following

Conjecture 1.4.1. There exists an equivalence of triangulated categories

$$
D^{\mathrm{GL}\left(V_{1}\right) \times \mathrm{GL}\left(V_{2}\right)} \mathrm{Coh}\left(\mathrm{St}_{\text {mir }}\right) \cong D_{\mathbf{I}}(\mathbf{F l} \times \mathbf{V}) .
$$

This conjecture would explain, in particular, the appearance of the same polynomials, called the Kostka-Shoji polynomials, in two different problems. On the one hand, it was proved in [FGT] that these polynomials are equal to the Poincaré polynomials of the stalks of $\mathbf{G}_{\mathbf{O}}$-equivariant IC sheaves on the mirabolic affine Grassmannian. On the other hand, it was proved in [FI] that the Kostka-Shoji polynomials are equal to the Poincaré polynomials of graded multiplicities of the natural $\mathrm{GL}_{N} \times \mathrm{GL}_{N}$-action on the space of global sections of line bundles on a convolution diagram of the cyclic $\widetilde{A}_{1}$-quiver.

1.5. Satake equivalence for some Lie supergroups. We consider the Lie superalgebra $\mathfrak{g l}(M \mid N)$ of endomorphisms of a super vector space $\mathbb{C}^{M \mid N}$, and the corresponding Lie supergroup $\operatorname{GL}(M \mid N)=\operatorname{Aut}\left(\mathbb{C}^{M \mid N}\right)$. We also consider a degenerate version $\mathfrak{g l}(M \mid N)$ where the supercommutator of the even elements (with even or od elements) is the same as in $\mathfrak{g l}(M \mid N)$, while

\footnotetext{
${ }^{2}$ Here we view both $\operatorname{Hom}\left(V_{1}, V_{2}\right)$ and $\operatorname{Hom}\left(V_{2}, V_{1}\right)$ as odd vector spaces, so that the functions on $\operatorname{Hom}\left(V_{1}, V_{2}\right)[1] \times \operatorname{Hom}\left(V_{2}, V_{1}\right)[1]$ (with grading disregarded) form really a symmetric (infinitedimensional) algebra, not an exterior algebra.
} 
the supercommutator of any two odd elements is set to be zero. In other words, the even part $\mathfrak{g l}(M \mid N)_{\overline{0}}=\mathfrak{g l}_{M} \oplus \mathfrak{g l}_{N}$ acts naturally on the odd part $\underline{\mathfrak{g l}}(M \mid N)_{\overline{1}}=\operatorname{Hom}\left(\mathbb{C}^{M}, \mathbb{C}^{N}\right) \oplus \operatorname{Hom}\left(\mathbb{C}^{N}, \mathbb{C}^{M}\right)$, but the supercommutator $\underline{\mathfrak{g l}}(M \mid N)_{\overline{1}} \times \underline{\mathfrak{g l}}(M \mid N)_{\overline{1}} \rightarrow \underline{\mathfrak{g l}}(M \mid N)_{\overline{0}}$ equals zero.

The category of finite dimensional representations of the corresponding supergroup $\underline{\mathrm{GL}}(M \mid N)$ (in vector superspaces) is denoted $\operatorname{Rep}(\underline{\mathrm{GL}}(M \mid N)$ ), and its bounded derived category is denoted $S D(\underline{\mathrm{GL}}(M \mid N))$.

There is a Koszul equivalence $\varkappa: S D(\underline{\mathrm{GL}}(N \mid N)) \stackrel{\sim}{\longrightarrow} S D_{\text {perf }}^{\mathrm{GL}_{N} \times \mathrm{GL}_{N}}\left(\mathfrak{S}_{1,1}^{\bullet}\right)$ (equivariant perfect dg-supermodules over dg-superalgebra $\left.\mathfrak{S}_{1,1}\right)$, see e.g. [MR]. It intertwines the usual tensor product of $\mathrm{GL}(N \mid N)$-modules with the tensor product $\otimes_{\mathfrak{S}_{1,1}}$ on $S D_{\text {perf }}^{\mathrm{GL}_{N} \times \mathrm{GL}_{N}}\left(\mathfrak{S}_{1,1}^{\bullet}\right)$. Composing the Koszul equivalence $\varkappa$ with the mirabolic Satake equivalence $\Phi^{1,1}: S D_{\text {perf }}^{\mathrm{GL}_{N} \times \mathrm{GL}_{N}}\left(\mathfrak{S}_{1,1}^{\bullet}\right) \stackrel{\sim}{\longrightarrow} S D_{! * \mathbf{G}_{\mathbf{O}}}(\mathbf{G r} \times \mathbf{V})($ constructible sheaves of supervector spaces) we obtain a super Satake equivalence $S D(\underline{\mathrm{GL}}(N \mid N)) \stackrel{\sim}{\longrightarrow} S D_{! * \mathbf{G}_{\mathbf{O}}}(\mathbf{G r} \times \mathbf{V})$ that intertwines the usual tensor product of $\underline{\mathrm{GL}}(N \mid N)$-modules with the fusion product on $S D_{! * \mathbf{G}_{\mathbf{O}}}(\mathbf{G r} \times \mathbf{V})$. Moreover, this derived equivalence is exact with respect to the tautological $t$-structure on $S D(\underline{\mathrm{GL}}(N \mid N))$ with the heart $\operatorname{Rep}(\underline{\mathrm{GL}}(N \mid N))$, resp. the perverse $t$-structure on $S D_{! * \mathbf{G}_{\mathbf{O}}}(\mathbf{G r} \times \mathbf{V})$ with the heart $S \operatorname{Perv}_{\mathbf{G}_{\mathbf{O}}}(\mathbf{G r} \times \mathbf{V})$.

Similarly, we construct equivalences

$$
S D(\underline{\mathrm{GL}}(N-1 \mid N)) \underset{\bar{\varkappa}}{\stackrel{\sim}{\rightarrow}} S D_{\text {perf }}^{\mathrm{GL}_{N-1} \times \mathrm{GL}_{N}}\left(\overline{\mathfrak{S}}_{1,1}^{\bullet}\right) \underset{\bar{\Phi}}{\stackrel{\sim}{\rightarrow}} S D_{\mathrm{GL}(N-1, \mathbf{O})}\left(\mathbf{G r}_{\mathrm{GL}_{N}}\right),
$$

where $\overline{\mathfrak{S}}_{1,1}^{\bullet}=\operatorname{Sym} \bullet\left(\operatorname{Hom}\left(\mathbb{C}^{N-1}, \mathbb{C}^{N}\right)[-1] \oplus \operatorname{Hom}\left(\mathbb{C}^{N}, \mathbb{C}^{N-1}\right)[-1]\right) .^{3} \quad$ The composition is again $t$-exact with respect to the tautological $t$-structure on $S D(\underline{\mathrm{GL}}(N-1 \mid N))$ with the heart $\operatorname{Rep}(\underline{\mathrm{GL}}(N-1 \mid N))$, resp. the perverse t-structure on $S D_{\mathrm{GL}(N-1, \mathbf{O})}\left(\mathbf{G r}_{\mathrm{GL}_{N}}\right)$ with the heart $S \operatorname{Perv} \mathrm{GL}(N-1, \mathbf{O})\left(\mathbf{G r}_{\mathrm{GL}_{N}}\right)$. Moreover, the composition intertwines the usual tensor product of $\underline{\mathrm{GL}}(N-1 \mid N)$-modules with the fusion product on $S \operatorname{Perv} \operatorname{GL}_{(N-1, \mathbf{O})}\left(\mathbf{G r}_{\mathrm{GL}_{N}}\right)$. Similarly to Section 1.3, the functor $\bar{\Phi}$ can be extended to an equivalence $\bar{\Phi}_{\hbar}: S D_{\text {perf }}^{\mathrm{GL}_{N-1} \times \mathrm{GL}_{N}}\left(\overline{\mathfrak{D}}^{\bullet}\right) \stackrel{\sim}{\sim} S D_{\mathrm{GL}(N-1, \mathbf{O}) \rtimes \mathbb{C}^{\times}}\left(\mathbf{G r}_{\mathrm{GL}_{N}}\right)$, where $\overline{\mathfrak{D}}^{\bullet}$ is the graded Weyl algebra of shifted differential operators on $\operatorname{Hom}\left(\mathbb{C}^{N-1}, \mathbb{C}^{N}\right)$ (with $\operatorname{deg} \hbar=2$ and all the other generators in degree 1).

1.6. Gaiotto conjectures. One may wonder if there is a geometric realization of categories of representations of nondegenerate supergroups $\mathrm{GL}(N \mid N), \mathrm{GL}(N-$ $1 \mid N)$. It turns out that such a realization exists (conjecturally) for the categories of integrable representations of quantized algebras $U_{q}(\mathfrak{g l}(N \mid N)), U_{q}(\mathfrak{g l}(N-1 \mid N))$.

\footnotetext{
${ }^{3}$ Here we view both $\operatorname{Hom}\left(\mathbb{C}^{N-1}, \mathbb{C}^{N}\right)$ and $\operatorname{Hom}\left(\mathbb{C}^{N}, \mathbb{C}^{N-1}\right)$ as odd vector spaces, so that $\operatorname{Sym} \bullet\left(\operatorname{Hom}\left(\mathbb{C}^{N-1}, \mathbb{C}^{N}\right)[-1] \oplus \operatorname{Hom}\left(\mathbb{C}^{N}, \mathbb{C}^{N-1}\right)[-1]\right)$ (with grading disregarded) is really a symmetric (infinite-dimensional) algebra, not an exterior algebra.
} 
First of all, similarly to the classical Kazhdan-Lusztig equivalence, it is expected that $U_{q}(\mathfrak{g l}(M \mid N))-\bmod \cong \mathrm{KL}_{c}(\widehat{\mathfrak{g l} l}(M \mid N))$, where $q=\exp (\pi \sqrt{-1} / c)$, and $\mathrm{KL}_{c}(\widehat{\mathfrak{g l}}(M \mid N))$ stands for the derived category of $\mathrm{GL}(M, \mathbf{O}) \times \mathrm{GL}(N, \mathbf{O})$ equivariant $\widehat{\mathfrak{g l}}(M \mid N)$-modules at the level corresponding to the invariant bilinear form $(X, Y)=c \cdot \operatorname{sTr}(X Y)-\frac{1}{2} \operatorname{Killing}_{\mathfrak{g l}(M \mid N)}(X, Y)$ on $\mathfrak{g l}(M \mid N)$. Second, it is expected that the category $\mathrm{KL}_{c}(\widehat{\mathfrak{g l}}(N-1 \mid N))$ is equivalent to the $q$-monodromic $\mathrm{GL}(N-1, \mathrm{O})$-equivariant derived constructible category of the complement $\mathcal{L} \bullet$ of the zero section of the determinant line bundle on $\mathbf{G r}_{\mathrm{GL}_{N}}$, and this equivalence takes the standard $t$-structure of $\mathrm{KL}_{c}(\widehat{\mathfrak{g l}}(N-1 \mid N))$ to the perverse $t$-structure.

Further, it is expected that $\mathrm{KL}_{c}(\widehat{\mathfrak{g l}}(N \mid N))$ is equivalent to the $q$-monodromic $\mathrm{GL}(N, \mathbf{O})$-equivariant derived constructible category of $\mathcal{L}^{\bullet} \times \mathbf{V}$, and this equivalence takes the standard $t$-structure of $\mathrm{KL}_{c}(\widehat{\mathfrak{g l}}(N \mid N))$ to the perverse $t$-structure. For $M<N-1$ it is expected that $\mathrm{KL}_{c}(\widehat{\mathfrak{g l} l}(M \mid N))$ is equivalent to the $q$-monodromic $\mathrm{GL}(M, \mathrm{O})$-equivariant derived constructible category of $\mathcal{L}^{\bullet}$ with certain Whittaker conditions, cf. Section 2.6 for more details. In particular, the special case $M=0$ of this conjecture follows from the Fundamental Local Equivalence [G1, G3, G4] of the geometric Langlands program.

There are similar expectations for other classical (i.e. orthosymplectic) Lie superalgebras; the interested reader may try to find them in [GW].

1.7. Conjectures of Ben-Zvi, Sakellaridis and Venkatesh. In an ongoing project of D. Ben-Zvi, Y. Sakellaridis and A. Venkatesh, the authors propose the Periods - $L$-functions duality conjectures. Their conjectures predict, among other things, that given a reductive group $G$ and its spherical homogeneous variety $X=\mathrm{G} / \mathrm{H}$, there is a subgroup $\mathrm{G}_{X}^{\vee} \subset \mathrm{G}^{\vee}$, its graded representation $V_{X}^{\vee}=$ $\bigoplus_{i \in \mathbb{Z}} V_{X, i}^{\vee}[i]$, and an equivalence $D \operatorname{Coh}\left(V_{X}^{\vee} / \mathrm{G}_{X}^{\vee}\right)=D \operatorname{Coh}\left(\left(\bigoplus_{i \in \mathbb{Z}} V_{X, i}^{\vee}[i]\right) / \mathrm{G}_{X}^{\vee}\right) \simeq$ $D_{\mathrm{G}(\mathbf{O})}(X(\mathbf{F}))$. For a partial list of examples, see the table at the end of [Sa]. The relevant representations $V_{X}^{\vee}$ (constructed in terms of the Luna diagram of $X$ ) can be read off from the 4-th column of the table.

It turns out that the equivalences discussed in Section 1.5 fit into the general setting outlined in the previous paragraph. Thus the case of Example 13 of [Sa] corresponds to the equivalence $\bar{\Phi}: D_{\text {perf }}^{\mathrm{GL}_{N-1} \times \mathrm{GL}_{N}}\left(\overline{\mathfrak{S}}_{1,1}^{\bullet}\right) \stackrel{\sim}{\longrightarrow} D_{\mathrm{GL}(N-1, \mathbf{O})}\left(\mathbf{G r}_{\mathrm{GL}_{N}}\right)$ of Section 1.5. To explain this, let $\mathrm{G}:=\mathrm{GL}_{N-1} \times \mathrm{GL}_{N}$ and $\mathrm{H}:=\mathrm{GL}_{N-1}$. We view $\mathrm{H}$ as a block-diagonal subgroup of $\mathrm{G}$ and put $X=\mathrm{G} / \mathrm{H}$. Then loosely speaking we have $D_{\mathrm{GL}(N-1, \mathbf{O})}\left(\mathbf{G r}_{\mathrm{GL}}\right) \simeq D(\mathrm{GL}(N-1, \mathbf{O}) \backslash \mathrm{GL}(N, \mathbf{F}) / \mathrm{GL}(N, \mathbf{O})) \simeq$ $D(\mathrm{G}(\mathbf{O}) \backslash \mathrm{G}(\mathbf{F}) / \mathrm{H}(\mathbf{F})) \simeq D(\mathrm{G}(\mathbf{O}) \backslash X(\mathbf{F})) \simeq D_{\mathrm{G}(\mathbf{O})}(X(\mathbf{F}))$. On the other hand, we consider a graded $\mathrm{G}^{\vee}$-module $V_{X}^{\vee}:=\operatorname{Hom}\left(\mathbb{C}^{N-1}, \mathbb{C}^{N}\right)[1] \oplus \operatorname{Hom}\left(\mathbb{C}^{N}, \mathbb{C}^{N-1}\right)[1]$ (similarly to the footnote in Section 1.5 , we view $V_{X}^{\vee}$ as an odd vector space placed in cohomological degree -1 . Note also that $\left.\mathrm{G}^{\vee} \simeq \mathrm{G}=\mathrm{GL}_{N-1} \times \mathrm{GL}_{N}\right)$. Hence, the equivalence $\bar{\Phi}$ of Section 1.5 takes the form $D \operatorname{Coh}\left(V_{X}^{\vee} / G^{\vee}\right) \simeq D_{\mathrm{G}(\mathbf{O})}(X(\mathbf{F}))$. 
Similarly, Example 14 of [Sa] matches the Gaiotto conjecture of Section 1.6 for an orthosymplectic Lie superalgebra.

1.8. Organization of the paper. In Section 2 (which is not necessary for understanding subsequent sections) we formulate the Gaiotto conjectures and explain their relation with the geometric Langlands program; in particular, with the Fundamental Local Equivalence. Section 3 is the technical core of the paper. In this section we establish a coherent description of the spherical mirabolic affine Hecke category $D_{\mathrm{GL}(N, \mathbf{O})}(\mathbf{G r} \times \mathbf{V})$ with its three monoidal structures. Also, as a preparation for Section 4 we give a coherent description of the restriction functor $D_{\mathrm{GL}(N, \mathbf{O})}(\mathbf{G r} \times \mathbf{V}) \rightarrow D_{\mathrm{GL}(N, \mathbf{O})}\left(\mathbf{G r} \times \mathbf{V}_{0}\right) .{ }^{4}$ In Section 4 we establish a coherent description of the category $D_{\mathrm{GL}(N-1, \mathbf{O})}(\mathbf{G r})$ along with its fusion monoidal structure. In Section 5 we prove the quantum analogues, that is, counterparts for categories equivariant with respect to the loop rotations, of the results above.

1.9. Acknowledgments. We are grateful to R. Bezrukavnikov, D. Ben-Zvi, I. Entova-Aizenbud, P. Etingof, B. Feigin, D. Gaiotto, D. Gaitsgory, J. Hilburn, D. Leites, C. Mautner, Y. Sakellaridis, V. Serganova, A. Venkatesh and P. Yoo for very useful discussions. A.B. was partially supported by NSERC. M.F. was partially funded within the framework of the HSE University Basic Research Program and the Russian Academic Excellence Project '5-100'. The work of V.G. was supported in part by an NSF award DMS-1602111.

\section{Gaiotto conjectures}

The purpose of the present Section is to put the main results of this paper into a wider framework which has to do with the local geometric Langlands correspondence. This section will be used for motivation purposes only. It can be safely skipped by the readers who are not interested in the geometric Langlands correspondence. The ideas of this Section are due to D. Gaiotto (private communication). Gaiotto informed us that his ideas were motivated, to a large extent, by his discussions with P. Yoo as well as by [MW].

2.1. Reminder on strong actions on categories. Let $G$ be a connected reductive group over $\mathbb{C}$ and let $\kappa$ denote an invariant symmetric bilinear form on the Lie algebra $\mathfrak{g}$ of $G$ (when $G$ is simple, the vector space of such bilinear forms is 1-dimensional, so we can think of $\kappa$ as an element of $\mathbb{C}$ ). Then there is a notion of strong action of the group $G_{\mathbf{F}}$ on a category $\mathcal{C}$ of level $\kappa$. We refer the reader to [G3] for details of the definition. It will be important for us later that this definition is, in some sense, invariant under integral shifts. Specifically, any category $\mathcal{C}$ endowed with a $G_{\mathbf{F}^{-}}$action of level $\kappa$ has a natural $G_{\mathbf{F}^{-}}$action of level

\footnotetext{
${ }^{4}$ Recall that $\mathbf{V}_{0}=V \llbracket t \rrbracket$ is the standard lattice in the Tate vector space $\mathbf{V}$.
} 
$\kappa+\kappa^{\prime}$, where $\kappa^{\prime}$ is another form as above which is, moreover, integral in the sense that the corresponding quadratic form is integral and even on elements of the coweight lattice of $G$. Here are two important examples:

1) Let $\hat{\mathfrak{g}}_{\kappa}$ denote the central extension of $\mathfrak{g}_{\mathbf{F}}$ associated with the form $\kappa$. Let $\hat{\mathfrak{g}}_{\kappa}$-mod be the category of continuous (with respect to $t$-adic topology) modules over $\hat{\mathfrak{g}}_{\kappa}$ such that the element 1 of the center acts on the module as the identity. Then, the adjoint action of $G_{\mathbf{F}}$ on $\hat{\mathfrak{g}}_{\kappa}$ has a natural lift to a strong action of $G_{\mathbf{F}}$ on $\hat{\mathfrak{g}}_{\kappa}$-mod of level $\kappa$. More generally, let $\mathfrak{a}=\mathfrak{a}_{\overline{0}} \oplus \mathfrak{a}_{\overline{1}}$ be a Lie superalgebra. Assume that:

(i) $G$ acts on $\mathfrak{a}$;

(ii) We are given a map $\iota: \mathfrak{g} \rightarrow \mathfrak{a}_{\overline{0}}$ such that the corresponding adjoint action of $\mathfrak{g}$ on $\mathfrak{a}$ is equal to the derivative of the $G$-action from (i).

(iii) The algebra $\mathfrak{a}$ is equipped with an invariant symmetric (in the super-sense) bilinear form $\kappa_{\mathfrak{a}}$. Let $\kappa_{\mathfrak{g}}$ be its pull-back to $\mathfrak{g}$.

Associated with the form $\kappa_{\mathfrak{a}}$, there is a canonical Kac-Moody extension $\hat{\mathfrak{a}}$ of $\mathfrak{a}_{\mathbf{F}}$. As before, we denote by $\hat{\mathfrak{a}}$-mod the category of continuous modules $M$ over $\hat{\mathfrak{a}}$ such that the element 1 of the center acts on $M$ by the identity. This category comes equipped with a $G_{\mathbf{F}}$-action of level $\kappa_{\mathfrak{g}}$.

We will mostly be interested in the following special case of the above construction. Fix a pair $M, N$, of non-negative integers. Let $\mathfrak{a}=\mathfrak{g l}(M \mid N), G=$ $\mathrm{GL}_{M} \times \mathrm{GL}_{N}$. Let $\kappa_{\mathfrak{a}}(x, y)=c \cdot \mathrm{s} \operatorname{Tr}(x y)$, where sTr stands for 'super-trace' and $c \in \mathbb{C}$.

2) Assume that the form $\kappa$ is integral and even. Then, this form gives rise to a central extension $\widehat{G}$ of $G_{\mathbf{F}}$. Let $X$ be an ind-scheme equipped with a $\widehat{G}$-equivariant line bundle $\mathcal{L}$. Then for any $c \in \mathbb{C}$ one has the category $D_{c}(X)$-mod of $c$-twisted $D$-modules on $X$. This category has a natural strong $G_{\mathbf{F}}$-action of level $c \cdot \kappa$.

\subsection{Digression on the local (quantum) geometric Langlands correspon-}

dence. Let $G$ and $\kappa$ be as above and assume in addition that the form $\kappa$ is nondegenerate. Let $G^{\vee}$ denote the Langlands dual group. Since $\kappa$ is non-degenerate it gives a similar form $\kappa^{\vee}$ for $G^{\vee}$. Further, put $\kappa_{\text {crit }}=-\frac{1}{2}$ Killing $_{\mathfrak{g}}$, where Killing stands for the Killing form on the Lie algebra $\mathfrak{g}$ of $G$.

The local quantum geometric Langlands conjecture is, roughly speaking, an equivalence of 2 -categories

$$
\text { \{Categories with strong } \left.G_{\mathbf{F}^{-}} \text {action of level } \kappa+\kappa_{\text {crit }}\right\}
$$

and

$$
\text { \{Categories with strong } \left.G_{\mathbf{F}^{-}}^{\vee} \text { action of level }-\kappa^{\vee}-\kappa_{\text {crit }}^{\vee}\right\} \text {. }
$$

The form $\kappa_{\text {crit }}$ being integral, the shift by $\kappa_{\text {crit }}$ in the above formulation is not very essential. However, it is convenient for many applications to make this shift. 
To give a rigorous meaning to the above conjecture one needs, first of all, to replace all 'categories' by suitable 'dg-categories'. This upgrades each side of the equivalence to an $(\infty, 2)$-category. Then for generic, i.e. non-rational, $\kappa$ the above equivalence is expected to hold as stated. For general $\kappa$, more corrections are necessary but we will not discuss this here since the local geometric Langlands correpondence will only serve as a guiding principle.

There is a "limiting version" of the above conjecture for $\kappa=0$. To explain this, we use the notion of a 'category over a stack $\mathcal{S}$ ', cf. [G3, §6] and references therein. Write $\mathcal{D}^{\circ}=\operatorname{Spec}(\mathbf{F})$ and let $\operatorname{LocSys}_{G^{\vee}}\left(\mathcal{D}^{\circ}\right)$ be the classifying stack of principal $G^{\vee}$-bundles on $\mathcal{D}^{\circ}$ equipped with a connection. The "classical" local geometric Langlands conjecture predicts a close relationship between

$$
\left\{\text { Categories with strong } G_{\mathbf{F}^{-}} \text {action of level } \kappa_{\text {crit }}\right\}
$$

and

$$
\left\{\text { Categories over } \operatorname{LocSys}_{G^{\vee}}\left(\mathcal{D}^{\circ}\right)\right\} \text {. }
$$

Here, $\kappa_{\text {crit }}$ can be replaced by 0 , since $\kappa_{\text {crit }}$ is integral. Again, it is possible to make the informal relation above a rigorous mathematical conjecture, cf. again $[\mathrm{G} 3, \S 6]$ for a more detailed discussion.

In the remaining part of this Section we will pretend that both quantum and classical geometric Langlands conjectures hold as stated. We will write $\mathcal{C} \mapsto \mathcal{C}^{\vee}$ for the resulting correspondence .

An important example of such a correspondence is as follows. Fix a nondegenerate form $\kappa$ and let $\mathcal{C}=D_{\kappa+\kappa_{\text {crit }}}\left(\mathbf{G r}_{G}\right)$-mod be the category of $\left(\kappa-\kappa_{\text {crit }}\right)$ twisted $D$-modules on the affine Grassmannian $\mathbf{G r}_{G}$ of $G$. It is expected that in this case the category $\mathcal{C}^{\vee}$ is the category $D_{-\kappa^{\vee}-\kappa_{\text {crit }}^{\vee}}\left(\mathbf{G r}_{G \vee}\right)$-mod. In the limiting case $\kappa=0$ category $\mathrm{C}^{\vee}$ is expected to be a push-forward of category QCoh(pt $/ G^{\vee}$ ) under the natural map pt $/ G^{\vee} \rightarrow \operatorname{LocSys}_{G^{\vee}}\left(\mathcal{D}^{\circ}\right)$ induced by the imbedding of the trivial local system. These expectations would imply the following:

(i) If $\kappa$ is non-degenerate, then $\mathfrak{e}^{G_{\mathbf{O}}} \simeq\left(\mathfrak{C}^{\vee}\right)^{G_{\mathbf{O}}^{\vee}}$ (here $\mathcal{C}^{G_{\mathbf{O}}}$ denotes the category of $G_{\mathbf{O}^{-}}$equivariant objects in $\mathcal{C}$ ).

(ii) If $\kappa=0$ then $\mathrm{e}^{G_{\mathbf{O}}}$ is equivalent to the pull-back of the category $\mathrm{C}^{\vee}$ under the map pt $/ G^{\vee} \rightarrow \operatorname{LocSys}_{G^{\vee}}\left(\mathcal{D}^{\circ}\right)$ corresponding to the trivial local system.

2.3. Whittaker category and the fundamental local equivalence (FLE). In this subsection we discuss another important example of Langlands dual categories. We refer to [G3] and [G4] for details.

Let $U$ be a maximal unipotent subgroup of $G$ and let $\chi_{0}: U \rightarrow \mathbb{G}_{a}$ be a nondegenerate character. We define a character $\chi: U_{\mathbf{F}} \rightarrow \mathbb{G}_{a}$ by

$$
\chi(u(t))=\operatorname{Res}_{t=0} \chi_{0}(u(t)) d t .
$$

Given a (co-complete, $\mathrm{dg}$ ) category $\mathcal{C}$ with a strong $G_{\mathbf{F}^{-}}$action of a fixed level one can define a category Whit $(\mathcal{C})$ of $\left(U_{\mathbf{F}}, \chi\right)$-equivariant objects in $\mathcal{C}$. 
We consider the category $D_{\kappa}\left(G_{\mathbf{F}}\right)$-mod of $\kappa$-twisted $D$-modules on $G_{\mathbf{F}}$. According to [AG1] this category has a natural action of $G_{\mathbf{F}}$ of level $\kappa$ that comes from left translations, and also another action of $G_{\mathbf{F}}$ of level $-\kappa+2 \kappa_{\text {crit }}$ that comes from right translations.

Let Whit $_{\kappa}^{r}\left(G_{\mathbf{F}}\right)$ denote its Whittaker category with respect to the right action. This category inherits the left action of level $\kappa$. One could ask what is its Langlands dual category.

For simplicity, below we will only consider either the case where $\kappa$ is not rational (i.e. the value of the corresponding quadratic form on any coroot is not a rational number) or the case $\kappa=0$.

Assume first that $\kappa$ is non-degenerate and not rational. Then it is expected (cf. [G3]) that

$$
\text { Whit }{ }_{\kappa-\kappa_{\text {crit }}}^{r}\left(G_{\mathbf{F}}\right)^{\vee} \text { is the category } \widehat{\mathfrak{g}}_{-\kappa^{\vee}+\kappa_{\text {crit }}^{\vee}}-\bmod \text {. }
$$

In the case $\kappa=0$ the expected answer is the category $\mathrm{QCoh}\left(\operatorname{LocSys}_{G^{\vee}}\left(\mathcal{D}^{\circ}\right)\right)$.

Let Whit ${ }_{\kappa}\left(\mathbf{G r}_{G}\right)$ denote the category of $\kappa$-twisted $D$-modules on $\mathbf{G r}_{G}$ and let $\mathrm{KL}_{\kappa}(\mathfrak{g})=\left(\hat{\mathfrak{g}}_{\kappa}-\mathrm{mod}\right)^{G_{\mathbf{O}}}$. Then, statements (i) and (ii) of Section 2.2 imply the following result, which has been proved rigorously in [G4] and [G1] (it goes under the name "fundamental local equivalence"):

Theorem 2.3.1. Assume that $\kappa$ is non-degenerate and not rational.

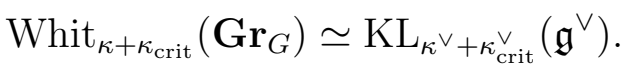

$$
\begin{aligned}
& \text { Whit }\left(\mathbf{G r}_{G}\right) \simeq \operatorname{Rep}\left(G^{\vee}\right) \text {. }
\end{aligned}
$$

Moreover, these equivalences hold on the level of abelian categories.

Remarks 2.3.2. 1) The critical shifts in (2.3.2) are not important for irrational $\kappa$ if one only cares about both sides as abstract categories. However, we still prefer to keep them, since in this way one can also extend the statement to rational $\kappa$; in addition the shifts are important if we keep track of some natural structures on these categories (cf. 4) below.

2) Note the absence of a negative sign before $\kappa^{\vee}+\kappa_{\text {crit }}^{\vee}$ in the RHS of (2.3.2). This has to do with the fact that Whit ${ }_{\kappa+\kappa_{\text {crit }}}\left(\mathbf{G r}_{G}\right)$ is actually the category of $G_{\mathbf{O}^{-}}$equivariant objects with respect to the left action of $G_{\mathbf{O}}$ on the category of $D$-modules on $G_{\mathbf{F}}$ which are Whittaker on the right. This change of right to left is what is responsible for the change of sign.

3) The fact that the above equivalences respect the natural t-structures does not follow (to the best of our knowledge) from any geometric Langlands considerations. In fact, at the level of (unbounded) derived categories the statement holds for all $\kappa$, but when $\kappa$ is positive rational it is very far from an abelian equivalence.

4) The above equivalences are in fact not only equivalences of abstract categories, but equivalences of categories with factorization structure, a notion closely 
related to the notion of a braided monoidal category (it is also worthwhile to note that as an abstract category, $\mathrm{KL}_{\kappa}(\hat{\mathfrak{g}})$ is independent of $\kappa$ if $\kappa$ is irrational; but it is not so if we take into account the factorization structure).

2.4. Gaiotto conjectures: geometric Langlands form in the case $N>M$. There is one more series of examples which are relevant for the subject of this paper. Fix two non-negative integers $N$ and $M$ such that $N \geq M$ and set $G_{M, N}=\mathrm{GL}_{M} \times \mathrm{GL}_{N}$. Note that $G_{M, N}$ is isomorphic to $G_{M, N}^{\vee}$.

We are going to produce an example of Langlands dual categories for $G_{M, N}$; for $M=0$ we will recover (2.3.1). First, we describe the analog of the RHS. Let $c \in \mathbb{C}$. Then the category in question will be the category $\widehat{\mathfrak{g l}}(M \mid N)_{c}$-mod of modules over the affine Lie super-algebra $\widehat{\mathfrak{g l}}(M \mid N)$ of level $c \cdot \kappa_{M, N}+\frac{1}{2} \operatorname{Killing}_{M, N}$, where

1) $\kappa_{M, N}(x, y)=\operatorname{sTr}(x y)$.

2) Killing ${ }_{M, N}$ is the restriction of the Killing form of $\mathfrak{g l}(M \mid N)$ to the even part (note that it is degenerate if $M=N$ ).

This category has a natural action of the group $G_{M, N}(\mathbf{F})$ of certain level which is an integral shift of $\left(c \cdot \kappa_{M},-c \cdot \kappa_{N}\right)$ (here $\kappa_{N}$ denotes the standard invariant bilinear form on the Lie algebra $\mathfrak{g l}_{N}$ equal to $\left.\operatorname{Tr}(X \cdot Y)\right)$. As has been explained above, one can twist the action of $G_{M, N}(\mathbf{F})$ on this category so that the twist becomes equal to $\left(c \cdot \kappa_{M},-c \cdot \kappa_{N}\right)-\kappa_{\text {crit }}$ (here by $\kappa_{\text {crit }}$ we mean the critical bilinear form for the Lie algebra $\left.\mathfrak{g}_{M, N}\right)$. Hence it makes sense to consider its Langlands dual. This should be a category with a strong action of $G_{M, N}(\mathbf{F})$ of level $c^{-1} \cdot\left(\kappa_{M},-\kappa_{N}\right)-\kappa_{\text {crit }}$. However, we can again twist the action and think of it as a category with an action of $G_{M, N}(\mathbf{F})$ of level $c^{-1} \cdot\left(\kappa_{M},-\kappa_{N}\right)$. Let us give a conjectural description of the Langlands dual category according to a prediction of D. Gaiotto.

Next, let $M<N$. We define a unipotent subgroup $U_{M, N}$ of $\mathrm{GL}_{N}$ as follows. If $M=N-1$ this subgroup is trivial, and if $M=0$ it is the group $U_{N}$ of unipotent upper-triangular matrices. In the general case, $U_{M, N}$ is a subgroup of $U_{N}$ defined as follows.

Let $e_{M, N} \in \mathfrak{g l}_{N}$ be the standard upper-triangular Jordan block of size $N-M$, i.e.

$$
e_{M, N}=\sum_{i=1}^{N-M-1} E_{i, i+1},
$$

where $E_{i j}$ stands for the matrix whose $(i, j)$-entry is equal to 1 and all other entries are equal to 0 . The element $e_{M, N}$ is part of an $\mathfrak{s l}_{2}$-triple $\left(e_{M, N}, h_{M, N}, f_{M, N}\right)$. Here $f_{M, N}=\sum_{i=1}^{N-M-1} i(N-M-i) E_{i+1, i}$ and $h_{M, N}$ is the diagonal matrix which has diagonal entries $(N-M-1, N-M-3, \ldots,-N+M+1,0, \ldots, 0)$. For any 
integer $l$ we let $\mathfrak{g}_{l}$ denote the $l$-eigen-space of the adjoint action of $h_{M, N}$ on $\mathfrak{g l}_{N}$. We set

$$
\mathfrak{u}_{M, N}=\bigoplus_{l \geq 2} \mathfrak{g}_{l} \oplus \mathfrak{g}_{1}^{+}
$$

where $\mathfrak{g}_{1}^{+}$is the intersection of $\mathfrak{g}_{1}$ with the Lie algebra of upper-triangular matrices. We define a Lie algebra homomorphism $\chi_{M, N}^{0}: \mathfrak{u}_{M, N} \rightarrow \mathbb{C}$ by sending a matrix $\left(u_{i j}\right)$ to $\sum_{i=1}^{N-M-1} u_{i, i+1}$. Let $U_{M, N}$ be a unipotent subgroup of $\mathrm{GL}_{N}$ with Lie algebra $\mathfrak{u}_{M, N}$ and let $U_{M, N} \rightarrow \mathbb{G}_{a}$ be the homomorphism induced by $\chi_{M, N}^{0}$ (which we denote by the same symbol $\left.\chi_{M, N}^{0}\right)$.

We imbed the group $\mathrm{GL}_{M}$ into the centralizer of the element $h_{M, N}$ in $\mathrm{GL}_{N}$ (this is the block-diagonal embedding corresponding to rows $N-M+1, \ldots, N$ ). It is easy to see that the group $\mathrm{GL}_{M}$ normalizes the subgroup $U_{M, N}$ and the homomorphism $\chi_{M, N}^{0}$ is fixed by the $\mathrm{GL}_{M}$-action on $U_{M, N}$ by conjugation.

Remark 2.4.1. $U_{M, N}$ is conjugate to the subgroup $U_{M, N}^{\prime}$ formed by the blockupper-triangular matrices $\left(\begin{array}{ccc}U_{r} & * & * \\ 0 & 1_{M+1} & * \\ 0 & 0 & U_{s}\end{array}\right)$ where

$$
r=\lceil(N-M-1) / 2\rceil, s=\lfloor(N-M-1) / 2\rfloor ;
$$

in particular $r+s=N-M-1$. Here $U_{p}$ stands for an arbitrary unipotent uppertriangular matrix in $\mathrm{GL}_{p}$, and the notation ' $*$ ' is used for arbitrary matrices of an appropriate size. Moreover, the conjugation can be chosen so that the character $\chi_{M, N}^{0}$ corresponds to the character $\chi_{M, N}^{(r, s)}$ on $\mathfrak{u}_{M, N}^{(r, s)}:=\operatorname{Lie} U_{M, N}^{(r, s)}$ given by $\left(u_{i j}\right) \mapsto \sum_{i=1}^{r-1} u_{i, i+1}+u_{r k}+u_{k, N-s+1}+\sum_{i=N-s+1}^{N-1} u_{i, i+1}$ for any choice of $k \in$ $\{r+1, \ldots, N-s\}$. The subgroup $U_{M, N}^{(r, s)} \subset \mathrm{GL}_{N}$ and the character $\chi_{M, N}^{(r, s)}$ are defined for arbitrary pair $(r, s) \in \mathbb{N}^{2}:=\mathbb{Z}_{>0}^{2}$ with $r+s=N-M-1$. (In the two extreme cases $\{r, s\}=\{0, N-M-1\}$, one of the middle terms in the formula for $\chi_{M, N}^{(r, s)}$ is undefined and should be omitted.) Moreover $\chi_{M, N}^{(r, s)}$ can be replaced by an arbitrary representative of the open $N_{\mathrm{GL}_{N}}\left(U_{M, N}^{(r, s)}\right)$-orbit in $\mathfrak{u}_{M, N}^{(r, s) *}$.

Remark 2.4.2. $U_{M, N}^{(0, N-M-1)}$ is also conjugate to the unipotent radical $U_{(M+1,1, \ldots, 1)}$ of the standard parabolic subgroup $P_{(M+1,1, \ldots, 1)}$ of $\mathrm{GL}_{N}$ corresponding to the partition $(M+1,1, \ldots, 1)$ of $N$. The character $\chi_{M, N}^{0}$ is conjugate to the restriction of the regular character $u \mapsto \sum_{i=1}^{N-1} u_{i, i+1}$ of the upper triangular subgroup to $U_{(M+1,1, \ldots, 1)}$, cf. [JPS, $\left.\S(2.11)\right]$ and [C, the beginning of $\S 2$ of Lecture 5].

As before, we define a homomorphism $\chi_{M, N}: U_{M, N}(\mathbf{F}) \rightarrow \mathbb{G}_{a}$ to be $\operatorname{Res}_{t=0} \chi_{M, N}^{0}$. For any $c^{\prime} \in \mathbb{C}$ we now consider the category $D_{c^{\prime}}(\operatorname{GL}(N, \mathbf{F}))^{U_{M, N}(\mathbf{F}), \chi_{M, N}}$. By definition this is the derived category of $D$-modules on $\operatorname{GL}(N, \mathbf{F})$ twisted by $c^{\prime} \cdot \kappa_{N}$ 
that are equivariant on the left with respect to $\left(U_{M, N}(\mathbf{F}), \chi_{M, N}\right)$. This category has a natural action of $\mathrm{GL}(M, \mathbf{F})$ of level $c^{\prime} \cdot \kappa_{M}$ coming from left multiplication and an action of $\mathrm{GL}(N, \mathbf{F})$ of level $-c^{\prime} \cdot \kappa_{M}-\mathrm{Killing}_{\mathfrak{g l}_{N}}$ coming from right multiplication (recall that Killing $\mathfrak{g l}_{N}$ denotes the Killing form on $\mathfrak{g l}_{N}$ ). As before, we can twist the second action to make it an action of level $-c^{\prime} \cdot \kappa_{M}$.

Remark 2.4.3. In fact, using Fourier transform for $D$-modules, one can show that replacing $(r, s)$ in Remark 2.4.1 with any pair of non-negative integers whose sum equals $N-M-1$ produces a category equivalent to $D_{c^{\prime}}(\operatorname{GL}(N, \mathbf{F}))^{U_{M, N}(\mathbf{F}), \chi_{M, N}}$.

We now take $c^{\prime}=1 / c$. Then according to a conjecture of D. Gaiotto the category $D_{1 / c}(\operatorname{GL}(N, \mathbf{F}))^{U_{M, N}(\mathbf{F}), \chi_{M, N}}$ is Langlands dual to $\widehat{\mathfrak{g l}}(M \mid N)_{c}$-mod.

We can also consider the limit $c \rightarrow \infty$. To simplify the discussion we will not do it now, but we will discuss it later when we pass to $G_{\mathbf{O}^{-}}$equivariant objects.

2.5. Gaiotto conjectures: geometric Langlands form for $N=M$. Let us also discuss the case $N=M$. In this case on the left we again take the same category $\widehat{\mathfrak{g l}}(N \mid N)_{c}$-mod of modules over the affine Lie super-algebra $\widehat{\mathfrak{g l}}(N \mid N)$ of level $c \cdot \kappa_{N, N}-\frac{1}{2} \mathrm{Killing}_{N, N}$ (note that the Killing form is zero for $N=M$ ). The Langlands dual category (according to Gaiotto) is the derived category $D_{1 / c}(\mathrm{GL}(N, \mathbf{F}) \times \mathbf{V})$ of $1 / c$-twisted $D$-modules on $\mathrm{GL}(N, \mathbf{F}) \times \mathbf{V}$ where:

1) The twisting is with respect to the first factor.

2) The two actions of $\operatorname{GL}(N, \mathbf{F})$ come from the diagonal action coming from the left action of $\mathrm{GL}(N, \mathbf{F})$ on the first factor and the natural action of $\mathrm{GL}(N, \mathbf{F})$ on the 2nd factor, and the right multiplication action $\operatorname{GL}(N, \mathbf{F})$ on the first factor.

2.6. Gaiotto conjectures: "simple-minded" form. The above statements are not really well-formulated mathematical conjectures, since the local geometric Langlands duality is not known at present. However, we can turn them into precise conjectures by using (i) at the end of subsection 2.2, i.e. we are going to take $G_{M, N}(\mathbf{O})$-invariants on both sides. We get the following conjectures:

Conjecture 2.6.1. Assume that $N>M$ and assume that $c \neq 0$ is not a rational number. The categories $D_{1 / c}^{\left(\mathrm{GL}(M, \mathbf{O}) \ltimes U_{M, N}(\mathbf{F}), \chi_{M, N}\right)}\left(\mathbf{G r}_{\mathrm{GL}_{N}}\right)$ and $\mathrm{KL}_{c}(\widehat{\mathfrak{g l}}(M \mid N))$ are equivalent as factorization categories. Here $\mathrm{KL}_{c}(\widehat{\mathfrak{g l}}(M \mid N))$ is the category of $G_{M, N}(\mathbf{O})$-equivariant objects in the category $\widehat{\mathfrak{g l}}(M \mid N)_{c}$-mod.

Similarly the category $D_{1 / c}^{\mathrm{GL}(N, \mathbf{O})}\left(\mathbf{G r}_{\mathrm{GL}_{N}} \times \mathbf{V}\right)$ is equivalent to $\mathrm{KL}_{c}(\widehat{\mathfrak{g l}}(N \mid N))$.

We now want to take the limit $c \rightarrow \infty$. In this case $1 / c$ goes to 0 and the category of $1 / c$-twisted $D$-modules in Conjecture 2.6.1 just becomes the category of usual $D$-modules. The $c \rightarrow \infty$ limit of the category $\mathrm{KL}_{c}(\widehat{\mathfrak{g l}}(M \mid N))$ is not canonically defined: one has to choose some nice extension of the corresponding 
family of categories from $\mathbb{A}^{1}$ to $\mathbb{P}^{1}$, cf. $[\mathrm{Z} 1, \S 6]$ or $[\mathrm{Z} 2, \S 4]$. Naively, one might think that the correct extension is just the category of representations of the supergroup $\mathrm{GL}(M \mid N)$. However, it turns out that this is not the right choice. Instead, one needs to consider the category of representations of the group $\operatorname{GL}(M \mid N)$ ) defined in Section 1.5. With these conventions one gets the following

Conjecture 2.6.2. Assume that $N>M$. Then the category $D^{\left(\mathrm{GL}(M, \mathbf{O}) \ltimes U_{M, N}(\mathbf{F}), \chi_{M, N}\right)}\left(\mathbf{G r}_{\mathrm{GL}_{N}}\right)$ is equivalent to the category of modules over the group $\mathrm{GL}(M \mid N))$.

Similarly, for $N=M$ the category $D^{\mathrm{GL}(N, \mathbf{O})}\left(\mathbf{G r}_{\mathrm{GL}_{N}} \times \mathbf{V}\right)$ is equivalent to the category of modules over the group $\mathrm{GL}(N \mid N))$.

These equivalences should hold for both derived and abelian categories.

In the present work we prove Conjecture 2.6.2 for $M=N$ and $M=N-1$.

\section{A Coherent Realization of $D_{\mathrm{GL}(N, \mathbf{O})}(\mathbf{G r} \times \mathbf{V})$}

3.1. Setup and notation. We follow the notation of [FGT]. Recall that $\mathbf{G r}=$ $\mathbf{G r}_{\mathrm{GL}_{N}}=\mathbf{G}_{\mathbf{F}} / \mathbf{G}_{\mathbf{O}}=\mathrm{GL}(N, \mathbf{F}) / \mathrm{GL}(N, \mathbf{O})$, where $\mathbf{F}=\mathbb{C}((t)) \supset \mathbb{C} \llbracket t \rrbracket=\mathbf{O}$. We consider a complex vector space $V$ with a basis $e_{1}, \ldots, e_{N}$. We set $\mathbf{V}=V \otimes \mathbf{F} \supset$ $V \otimes \mathbf{O}=\mathbf{V}_{0}$.

Recall that the $\mathbf{G}_{\mathbf{F}}$-orbits in $\mathbf{G r} \times \mathbf{G r}$ (resp. in $\mathbf{G r} \times \mathbf{G r} \times \stackrel{\circ}{\mathbf{V}}$ ) are numbered in [FGT, Section 3.1] by signatures ${ }^{5}$ (resp. by bisignatures, i.e. pairs of signatures)

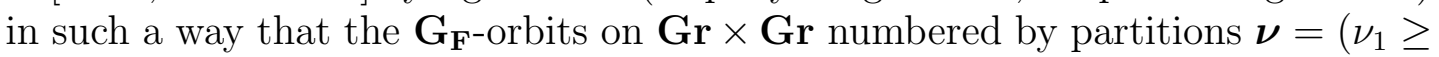
$\left.\ldots \geq \nu_{N} \geq 0\right)$ correspond to the pairs of lattices $L^{1} \subset L^{2}$. More precisely, the orbit corresponding to a bisignature $(\boldsymbol{\lambda}, \boldsymbol{\mu})$ contains a point

$\left(L^{1}=\mathbf{O}\left\langle e_{1}, e_{2}, \ldots, e_{N}\right\rangle, L^{2}=\mathbf{O}\left\langle t^{-\lambda_{1}-\mu_{1}} e_{1}, t^{-\lambda_{2}-\mu_{2}} e_{2}, \ldots, t^{-\lambda_{N}-\mu_{N}} e_{N}\right\rangle, v=\sum_{i=1}^{N} t^{-\lambda_{i}} e_{i}\right)$.

Irreducible representations of $\mathrm{GL}_{N}=\mathrm{GL}(V)$ are also numbered by the signatures, so that e.g. the determinant character $\operatorname{det} V$ corresponds to $\left(1^{N}\right)$. To a signature $\boldsymbol{\nu}=\left(\nu_{1} \geq \ldots \geq \nu_{N}\right)$ we associate an irreducible representation $V_{\boldsymbol{\nu}}$ with the highest weight $\boldsymbol{\nu}$. The geometric Satake equivalence takes the irreducible perverse sheaf $\mathrm{IC}_{\boldsymbol{\nu}}$ to the irreducible representation $V_{\nu}^{*}$. Thus if $\boldsymbol{\nu}$ is a partition (resp. a negative partition $\left.\left(0 \geq \nu_{1} \geq \ldots \geq \nu_{N}\right)\right)$, then $V_{\nu}^{*}$ is an antipolynomial (resp. polynomial) representation of $\mathrm{GL}_{N}$ (a polynomial functor in $V^{*}, V$ respectively).

A word of apology for our weird convention is in order. The numbering of $\mathbf{G}_{\mathbf{O}^{-}}$orbits in $\mathbf{G r}$ such that the orbits of sublattices $L \subset \mathbf{V}_{0}$ are numbered by partitions goes back at least to $[\mathrm{L}]$. We choose the numbering such that the orbits of sublattices are numbered by negative partitions since under this numbering the adjacency order of $\mathbf{G}_{\mathbf{O}^{-}}$orbits in $\mathbf{G r} \times \stackrel{\circ}{\mathbf{V}}$ goes to Shoji's order [Sh], [FGT,

\footnotetext{
${ }^{5}$ sequences of integers $\boldsymbol{\nu}=\left(\nu_{1} \geq \ldots \geq \nu_{N}\right)$, following the terminology of $\mathrm{H}$. Weyl.
} 
Proposition 12] on the set of bisignatures. Furthermore, we choose the Satake equivalence $\mathrm{IC}_{\boldsymbol{\nu}} \mapsto V_{\nu}^{*}$ (as opposed to $\mathrm{IC}_{\boldsymbol{\nu}} \mapsto V_{\boldsymbol{\nu}}$ ) since it makes the statement of our main result Theorem 3.6.1 more neat.

3.2. Constructible mirabolic category and convolutions. The triangulated category $D_{\mathbf{G}_{\mathbf{O}}}(\mathbf{G r} \times \stackrel{\circ}{\mathbf{V}})$ is defined as in [FGT, Section 2.6]. We will denote it by $D_{* \mathbf{G}_{\mathbf{O}}}(\mathbf{G r} \times \mathbf{V})$. Recall that an object $\mathcal{F}$ of $D_{* \mathbf{G}_{\mathbf{O}}}(\mathbf{G r} \times \mathbf{V})$ is supported on $\mathbf{G r} \times t^{m} \mathbf{V}_{0}$ for certain $m \in \mathbb{Z}$, and there exists $n>m$ and a $\mathbf{G}_{\mathbf{O}}$-equivariant sheaf $\mathcal{F}_{n}$ on $\mathbf{G r} \times\left(t^{m} \mathbf{V}_{0} / t^{n} \mathbf{V}_{0}\right)$ such that $\mathcal{F}=p_{n}^{*} \mathcal{F}_{n}$, where

$$
p_{n}: \mathbf{G r} \times t^{m} \mathbf{V}_{0} \rightarrow \mathbf{G r} \times\left(t^{m} \mathbf{V}_{0} / t^{n} \mathbf{V}_{0}\right)
$$

is the natural projection. In other words, $\mathcal{F}$ is a collection of $\mathbf{G}_{\mathbf{O}^{-}}$-equivariant sheaves $\mathcal{F}_{n^{\prime}}$ on $\mathbf{G r} \times\left(t^{m} \mathbf{V}_{0} / t^{n^{\prime}} \mathbf{V}_{0}\right)$ for $n^{\prime} \geq n$ along with a compatible system of isomorphisms $p_{n^{\prime \prime} / n^{\prime}}^{*} \mathcal{F}_{n^{\prime}} \stackrel{\sim}{\longrightarrow} \mathcal{F}_{n^{\prime \prime}}$ for $n^{\prime \prime} \geq n^{\prime}$, where

$$
p_{n^{\prime \prime} / n^{\prime}}: \mathbf{G r} \times t^{m} \mathbf{V}_{0} / t^{n^{\prime \prime}} \mathbf{V}_{0} \rightarrow \mathbf{G r} \times t^{m} \mathbf{V}_{0} / t^{n^{\prime}} \mathbf{V}_{0}
$$

are the natural projections.

If we replace in the above definition $p_{n}^{*}$ by $p_{n}^{!}$and $p_{n^{\prime \prime} / n^{\prime}}^{*}$ by $p_{n^{\prime \prime} / n^{\prime}}^{!}$, then we obtain a triangulated category $D_{! \mathbf{G}_{\mathbf{O}}}(\mathbf{G r} \times \mathbf{V})$. Note that $p_{n^{\prime \prime} / n^{\prime}}$ is a smooth morphism of relative dimension $N\left(n^{\prime \prime}-n^{\prime}\right)$, so we have a canonical isomorphism $p_{n^{\prime \prime} / n^{\prime}}^{!} \cong p_{n^{\prime \prime} / n^{\prime}}^{*}\left[2 N\left(n^{\prime \prime}-n^{\prime}\right)\right]$. We also consider the intermediate version

$$
p_{n^{\prime \prime} / n^{\prime}}^{! *}:=p_{n^{\prime \prime} / n^{\prime}}^{*}\left[N\left(n^{\prime \prime}-n^{\prime}\right)\right]=p_{n^{\prime \prime} / n^{\prime}}^{!}\left[-N\left(n^{\prime \prime}-n^{\prime}\right)\right]
$$

exact for the perverse $t$-structure. The corresponding triangulated category will be denoted $D_{! * \mathbf{G}_{\mathbf{O}}}(\mathbf{G r} \times \mathbf{V})$.

We will make use of an identification $\mathbf{G r} \times \mathbf{V}=\mathbf{G}_{\mathbf{F}} \stackrel{\mathbf{G}_{\mathbf{O}}}{\times} \mathbf{V}=\left(\mathbf{G}_{\mathbf{F}} \times \mathbf{V}\right) / \mathbf{G}_{\mathbf{O}}$ (quotient with respect to the diagonal right-left action). We will denote the orbit of $(g, v) \in \mathbf{G}_{\mathbf{F}} \times \mathbf{V}$ by $[g, v]$. Note that the left diagonal action of $\mathbf{G}_{\mathbf{O}}$ on $\mathbf{G r} \times \mathbf{V}$ in terms of the above identification is $h \cdot[g, v]=[h g, v]$. We consider the following convolution diagram:

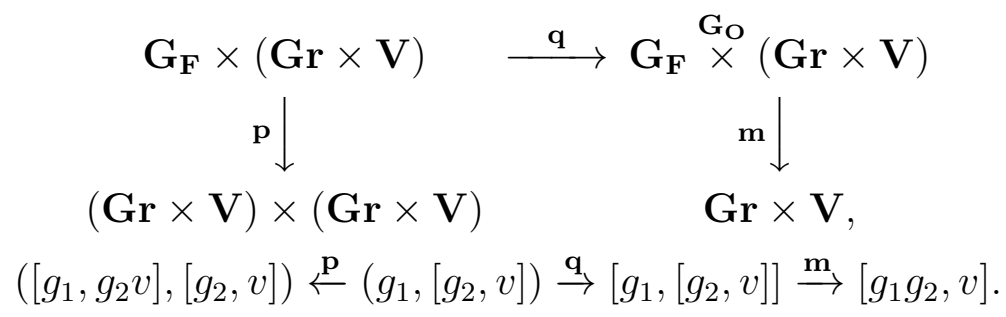

Given $\mathcal{F}_{1}, \mathcal{F}_{2} \in D_{! \mathbf{G}_{\mathbf{O}}}(\mathbf{G r} \times \mathbf{V})$, we define $\mathcal{F}_{1} \stackrel{!}{\circledast} \mathcal{F}_{2}:=\mathbf{m}_{*}\left(\mathcal{F}_{1} \widetilde{\otimes}^{!} \mathcal{F}_{2}\right)$, where $\mathcal{F}_{1} \widetilde{\otimes} ! \mathcal{F}_{2} \in D_{! \mathbf{G}_{\mathbf{O}}}\left(\mathbf{G}_{\mathbf{F}} \stackrel{\mathbf{G}_{\mathbf{O}}}{\times}(\mathbf{G r} \times \mathbf{V})\right)$ is the canonical descent of $\mathbf{p} !\left(\mathcal{F}_{1} \otimes \mathcal{F}_{2}\right)$ along $\mathbf{q}$. Similarly, given $\mathcal{F}_{1}, \mathcal{F}_{2} \in D_{* \mathbf{G}_{\mathbf{O}}}(\mathbf{G r} \times \mathbf{V})$, we define $\mathcal{F}_{1} \stackrel{*}{*} \mathcal{F}_{2}:=\mathbf{m}_{*}\left(\mathcal{F}_{1} \widetilde{\otimes}^{*} \mathcal{F}_{2}\right)$, 
where $\mathcal{F}_{1} \widetilde{\otimes}^{*} \mathcal{F}_{2} \in D_{* \mathbf{G}_{\mathbf{O}}}\left(\mathbf{G}_{\mathbf{F}} \stackrel{\mathbf{G}_{\mathbf{O}}}{\times}(\mathbf{G r} \times \mathbf{V})\right)$ is the canonical descent of $\mathbf{p}^{*}\left(\mathcal{F}_{1} \otimes \mathcal{F}_{2}\right)$ along $\mathbf{q}$. a unique sheaf such that $\mathbf{p}^{*}\left(\mathcal{F}_{1} \otimes \mathcal{F}_{2}\right)=\mathbf{q}^{*}\left(\mathcal{F}_{1} \widetilde{\otimes}^{*} \mathcal{F}_{2}\right)$.

We also consider another convolution diagram

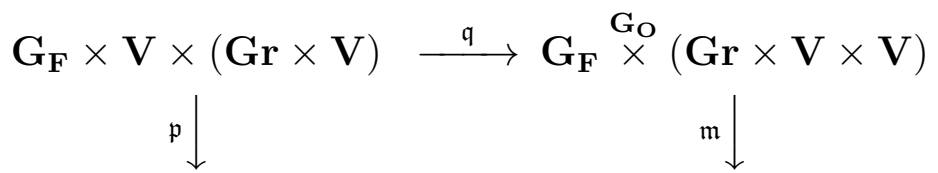

$$
(\mathbf{G r} \times \mathbf{V}) \times(\mathbf{G r} \times \mathbf{V}) \quad \mathbf{G r} \times \mathbf{V},
$$

$$
\left(\left[g_{1}, v_{1}\right],\left[g_{2}, v_{2}\right]\right) \stackrel{\mathfrak{p}}{\leftarrow}\left(g_{1}, v_{1},\left[g_{2}, v_{2}\right]\right) \stackrel{\mathfrak{q}}{\rightarrow}\left[g_{1},\left[g_{2}, g_{2}^{-1} v_{1}, v_{2}\right]\right] \stackrel{\mathfrak{m}}{\rightarrow}\left[g_{1} g_{2}, g_{2}^{-1} v_{1}+v_{2}\right] .
$$

Given $\mathcal{F}_{1}, \mathcal{F}_{2} \in D_{! \mathbf{G}_{\mathbf{O}}}(\mathbf{G r} \times \mathbf{V})$, we define $\mathcal{F}_{1} \stackrel{!}{*}^{\mathcal{F}_{2}}:=\mathfrak{m}_{!}\left(\mathcal{F}_{1} \widetilde{\otimes}^{!} \mathcal{F}_{2}\right)$, where $\mathcal{F}_{1} \widetilde{\otimes}^{!} \mathcal{F}_{2} \in$ $D_{! \mathbf{G}_{\mathbf{O}}}\left(\mathbf{G}_{\mathbf{F}} \stackrel{\mathbf{G}_{\mathbf{O}}}{\times}(\mathbf{G r} \times \mathbf{V} \times \mathbf{V})\right)$ is the canonical descent of $\mathfrak{p}^{!}\left(\mathcal{F}_{1} \otimes \mathcal{F}_{2}\right)$ along $\mathfrak{q}$. Similarly, given $\mathcal{F}_{1}, \mathcal{F}_{2} \in D_{* \mathbf{G}_{\mathbf{O}}}(\mathbf{G r} \times \mathbf{V})$, we define $\mathcal{F}_{1} \stackrel{*}{*} \mathcal{F}_{2}:=\mathfrak{m}_{*}\left(\mathcal{F}_{1} \widetilde{\otimes}^{*} \mathcal{F}_{2}\right)$, where $\mathcal{F}_{1} \widetilde{\otimes}^{*} \mathcal{F}_{2} \in D_{* \mathbf{G}_{\mathbf{O}}}\left(\mathbf{G}_{\mathbf{F}} \stackrel{\mathbf{G}_{\mathbf{O}}}{\times}(\mathbf{G r} \times \mathbf{V} \times \mathbf{V})\right)$ is the canonical descent of $\mathfrak{p}^{*}\left(\mathcal{F}_{1} \otimes \mathcal{F}_{2}\right)$ along $\mathfrak{q}$.

If we formally put $v_{1}=0$ (resp. $v_{2}=0$ ) in (3.2.2), we obtain the convolution diagrams

$$
\begin{aligned}
& \mathrm{G}_{\mathbf{F}} \times \mathrm{Gr} \times \mathrm{V} \stackrel{\mathfrak{q}}{\longrightarrow} \mathrm{G}_{\mathbf{F}} \stackrel{\mathrm{G}_{0}}{\times}(\mathrm{Gr} \times \mathrm{V}) \\
& \mathfrak{p}_{\text {left }} \downarrow \\
& \mathbf{G r} \times(\mathbf{G r} \times \mathbf{V}) \quad \mathbf{G r} \times \mathbf{V}, \\
& \left(\left[g_{1}\right],\left[g_{2}, v\right]\right) \stackrel{\mathfrak{p}_{\text {left }}}{\longleftarrow}\left(g_{1},\left[g_{2}, v\right]\right) \stackrel{\mathfrak{q}}{\rightarrow}\left[g_{1},\left[g_{2}, v\right]\right] \stackrel{\mathfrak{m}}{\rightarrow}\left[g_{1} g_{2}, v\right], \text { resp } \\
& \mathrm{G}_{\mathbf{F}} \times \mathrm{V} \times \mathrm{Gr} \stackrel{\mathfrak{q}}{\longrightarrow} \mathrm{G}_{\mathrm{F}} \stackrel{\mathrm{G}_{\mathrm{O}}}{\times}(\mathrm{Gr} \times \mathrm{V}) \\
& \mathfrak{p}_{\text {right }} \downarrow \mathfrak{m} \downarrow \\
& (\mathbf{G r} \times \mathbf{V}) \times \mathbf{G r} \quad \mathbf{G r} \times \mathbf{V}, \\
& \left(\left[g_{1}, v\right],\left[g_{2}\right]\right) \stackrel{\mathfrak{p}_{\text {right }}}{\longleftarrow}\left(g_{1}, v,\left[g_{2}\right]\right) \stackrel{\mathfrak{q}}{\rightarrow}\left[g_{1},\left[g_{2}, g_{2}^{-1} v\right]\right] \stackrel{\mathfrak{m}}{\rightarrow}\left[g_{1} g_{2}, g_{2}^{-1} v\right] .
\end{aligned}
$$

Given $\mathcal{P} \in D_{\mathbf{G}_{\mathbf{O}}}(\mathbf{G r})$ and $\mathcal{F} \in D_{? \mathbf{G}_{\mathbf{O}}}(\mathbf{G r} \times \mathbf{V})$ (where ? =!, *,!*), we define $\mathcal{P} * \mathcal{F}:=\mathfrak{m}_{*}(\mathcal{P} \widetilde{\otimes} \mathcal{F})$, where $\mathcal{P} \widetilde{\otimes} \mathcal{F} \in D_{?} \mathbf{G}_{\mathbf{O}}\left(\mathbf{G}_{\mathbf{F}} \stackrel{\mathbf{G}_{\mathbf{O}}}{\times}(\mathbf{G r} \times \mathbf{V})\right)$ is the canonical descent of $\mathfrak{p}_{\text {left }}^{*}(\mathcal{P} \otimes \mathcal{F})$ along $\mathfrak{q}$. We also define $\mathcal{F} * \mathcal{P}:=\mathfrak{m}_{*}(\mathcal{F} \widetilde{\otimes} \mathcal{P})$, where $\mathcal{F} \widetilde{\otimes} \mathcal{P} \in D_{? \mathbf{G}_{\mathbf{O}}}\left(\mathbf{G}_{\mathbf{F}} \stackrel{\mathbf{G}_{\mathbf{O}}}{\times}(\mathbf{G r} \times \mathbf{V})\right)$ is the canonical descent of $\mathfrak{p}_{\text {right }}^{*}(\mathcal{F} \otimes \mathcal{P})$ along $\mathfrak{q}$. Both the left and right convolutions are bi-exact for the perverse $t$-structures on $D_{\mathbf{G}_{\mathbf{O}}}(\mathbf{G r})$ and $D_{! * \mathbf{G}_{\mathbf{O}}}(\mathbf{G r} \times \mathbf{V})$, see [FGT, Section 3.9]. 
3.3. Fusion. Let $X$ be a smooth curve. For any integer $k>0$, and a collection $x=\left(x_{i}\right)_{i=1}^{k}$ of $S$-points of $X$, we denote by $\mathcal{D}_{x}$ the formal neighborhood of the union of graphs $|x|:=\bigcup_{i=1}^{k} \Gamma_{x_{i}} \subset S \times X$, and we denote by $\mathcal{D}_{x}^{\circ}:=\mathcal{D}_{x} \backslash|x|$ the punctured formal neighborhood. The mirabolic version of the Beilinson-Drinfeld Grassmannian is the ind-scheme $\mathbf{G r}_{B D, k}^{\mathrm{mir}}$ over $X^{k}$ parametrizing the following collections of data:

$$
\left(x_{i}\right)_{i=1}^{k}, \mathcal{E}, \phi:\left.\left.\mathcal{E}_{\text {triv }}\right|_{\mathcal{D}_{x}^{\circ}} \stackrel{\sim}{\sim} \mathcal{E}\right|_{\mathcal{D}_{x}^{\circ}}, v \in \Gamma\left(\mathcal{D}_{x}^{\circ}, \mathcal{E}\right)
$$

where $\mathcal{E}$ is a rank $N$ vector bundle on $\mathcal{D}_{x}$. In case $X=\mathbb{A}^{1}$, over the complement to the diagonals we have a canonical isomorphism

$$
\left(\mathbb{A}^{k} \backslash \Delta\right) \times_{\mathbb{A}^{k}} \mathbf{G r}_{B D, k}^{\mathrm{mir}} \cong\left(\mathbb{A}^{k} \backslash \Delta\right) \times(\mathbf{G r} \times \mathbf{V})^{k}
$$

We denote the projection $\left(\mathbb{A}^{k} \backslash \Delta\right) \times(\mathbf{G r} \times \mathbf{V})^{k} \rightarrow(\mathbf{G r} \times \mathbf{V})^{k}$ by $\mathrm{pr}_{2}$. Given $\mathcal{F}_{1}, \mathcal{F}_{2} \in D_{? \mathbf{G}_{\mathbf{O}}}(\mathbf{G r} \times \mathbf{V})$ (where ? =!,*,!*) we take $k=2$ and define the fusion

$$
\mathcal{F}_{1} \star \mathcal{F}_{2}:=\operatorname{pr}_{2 *} \psi_{x-y} \operatorname{pr}_{2}^{*}\left(\mathcal{F}_{1} \otimes \mathcal{F}_{2}\right)[1],
$$

where $x, y$ are coordinates on $\mathbb{A}^{2}$ (so that $x-y=0$ is the equation of the diagonal $\Delta \subset \mathbb{A}^{2}$ ), and $\psi_{x-y}$ is the nearby cycles functor for the pullback of the function $x-y$ to $\mathbf{G r}_{B D, 2}^{\mathrm{mir}}$, normalized so as to preserve the perverse $t$-structure. Note that the leftmost occurence of $\mathrm{pr}_{2}$ in the above definition projects $\mathbb{A}^{1} \times \mathbf{G r} \times \mathbf{V}$ to $\mathbf{G r} \times \mathbf{V}$, while the rightmost occurence of $\operatorname{pr}_{2}$ projects $\left(\mathbb{A}^{2} \backslash \Delta\right) \times(\mathbf{G r} \times \mathbf{V})^{2}$ to $(\mathbf{G r} \times \mathbf{V})^{2}$.

3.4. Coherent mirabolic category and convolutions. We write $\Pi E$ for an odd vector space obtained from a vector space $E$ by reversing the parity.

We fix a pair of $N$-dimensional vector spaces $V_{1} \simeq \mathbb{C}^{N} \simeq V_{2}$. We consider the Lie superalgebra $\mathfrak{g l}(N \mid N)=\mathfrak{g l}\left(V_{1} \oplus \Pi V_{2}\right)$. We have $\mathfrak{g l}(N \mid N)=\mathfrak{g}_{\overline{0}} \oplus \mathfrak{g}_{\overline{1}}$, where $\mathfrak{g}_{\overline{1}}=\Pi \operatorname{Hom}\left(V_{1}, V_{2}\right) \oplus \Pi \operatorname{Hom}\left(V_{2}, V_{1}\right)$, and $\mathfrak{g}_{\overline{0}}=\operatorname{End}\left(V_{1}\right) \oplus \operatorname{End}\left(V_{2}\right)$. We set $G_{\overline{0}}=$ $\mathrm{GL}\left(V_{1}\right) \times \mathrm{GL}\left(V_{2}\right)$. We consider the dg-algebra ${ }^{6} \mathfrak{G}_{1,1}^{\bullet}=\operatorname{Sym}\left(\mathfrak{g}_{1}[-1]\right)$ with zero differential, and the triangulated category $D_{\text {perf }}^{G_{\overline{0}}}\left(\mathfrak{G}_{1,1}^{\bullet}\right)$ obtained by localization (with respect to quasi-isomorphisms) of the category of perfect $G_{\overline{0}}$-equivariant dg- $\mathfrak{G}_{1,1}^{\bullet}$-modules. The category $D_{\text {perf }}^{G_{\overline{0}}}\left(\mathfrak{G}_{1,1}^{\bullet}\right)$ is monoidal with respect to $\mathcal{M}, \mathcal{M}^{\prime} \mapsto$ $\mathcal{M} \otimes_{\mathfrak{G}_{i, 1}} \mathcal{M}^{\prime}$, see Section 3.7 below.

We will also need two more versions of $\mathfrak{G}_{1,1}^{\bullet}$, namely

$$
\begin{aligned}
\mathfrak{G}_{0,2}^{\bullet} & =\operatorname{Sym}\left(\operatorname{Hom}\left(V_{1}, V_{2}\right)\right) \otimes \operatorname{Sym}\left(\operatorname{Hom}\left(V_{2}, V_{1}\right)[-2]\right), \\
\mathfrak{G}_{2,0}^{\bullet} & =\operatorname{Sym}\left(\operatorname{Hom}\left(V_{1}, V_{2}\right)[-2]\right) \otimes \operatorname{Sym}\left(\operatorname{Hom}\left(V_{2}, V_{1}\right)\right),
\end{aligned}
$$

and the corresponding triangulated categories $D_{\text {perf }}^{G_{\overline{0}}}\left(\mathfrak{G}_{0,2}^{\bullet}\right)$ and $D_{\text {perf }}^{G_{\overline{0}}}\left(\mathfrak{G}_{2,0}^{\bullet}\right)$. Now we will define the monoidal structures on $D_{\text {perf }}^{G_{\overline{0}}}\left(\mathfrak{G}_{0,2}^{\bullet}\right)$ and $D_{\text {perf }}^{G_{\overline{0}}}\left(\mathfrak{G}_{2,0}^{\bullet}\right)$.

\footnotetext{
${ }^{6}$ We view $\mathfrak{g}_{\overline{1}}$ as an odd vector space, so that $\operatorname{Sym}\left(\mathfrak{g}_{\overline{1}}[-1]\right)$ (with grading disregarded) is really a symmetric (infinite-dimensional) algebra, not an exterior algebra.
} 
We consider the variety $Q^{A}$ (resp. $\left.Q^{B}\right)$ of sixtuples

$$
\begin{gathered}
A \in \operatorname{Hom}\left(V_{1}, V_{2}\right), \quad B \in \operatorname{Hom}\left(V_{2}, V_{1}\right), A^{\prime} \in \operatorname{Hom}\left(V_{1}^{\prime}, V_{2}\right), \\
B^{\prime} \in \operatorname{Hom}\left(V_{2}, V_{1}^{\prime}\right), A^{\prime \prime} \in \operatorname{Hom}\left(V_{1}, V_{1}^{\prime}\right), B^{\prime \prime} \in \operatorname{Hom}\left(V_{1}^{\prime}, V_{1}\right),
\end{gathered}
$$

such that

$$
A=A^{\prime} A^{\prime \prime}, B^{\prime}=A^{\prime \prime} B, B^{\prime \prime}=B A^{\prime}\left(\operatorname{resp} . B=B^{\prime \prime} B^{\prime}, A^{\prime}=A B^{\prime \prime}, A^{\prime \prime}=B^{\prime} A\right)
$$

(here $V_{1}^{\prime}$ is a copy of $\left.V_{1}\right)$. Clearly, $Q^{A} \simeq \operatorname{Hom}\left(V_{1}, V_{1}^{\prime}\right) \times \operatorname{Hom}\left(V_{1}^{\prime}, V_{2}\right) \times \operatorname{Hom}\left(V_{2}, V_{1}\right)$, and $Q^{B} \simeq \operatorname{Hom}\left(V_{1}^{\prime}, V_{1}\right) \times \operatorname{Hom}\left(V_{2}, V_{1}^{\prime}\right) \times \operatorname{Hom}\left(V_{1}, V_{2}\right)$.

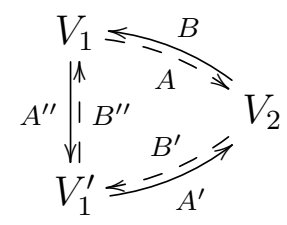

We denote $\operatorname{Hom}\left(V_{1}, V_{2}\right) \times \operatorname{Hom}\left(V_{2}, V_{1}\right) \times \operatorname{Hom}\left(V_{1}^{\prime}, V_{2}\right) \times \operatorname{Hom}\left(V_{2}, V_{1}^{\prime}\right) \times$ $\operatorname{Hom}\left(V_{1}, V_{1}^{\prime}\right) \times \operatorname{Hom}\left(V_{1}^{\prime}, V_{1}\right)$ by $\mathcal{H}$. We have the natural projections

$$
\begin{aligned}
\operatorname{pr}_{12}: \mathcal{H} \rightarrow \operatorname{Hom}\left(V_{1}, V_{2}\right) \times \operatorname{Hom}\left(V_{2}, V_{1}\right), \operatorname{pr}_{1^{\prime} 2}: \mathcal{H} \rightarrow \operatorname{Hom}\left(V_{1}^{\prime}, V_{2}\right) \times \operatorname{Hom}\left(V_{2}, V_{1}^{\prime}\right), \\
\operatorname{pr}_{11^{\prime}}: \mathcal{H} \rightarrow \operatorname{Hom}\left(V_{1}, V_{1}^{\prime}\right) \times \operatorname{Hom}\left(V_{1}^{\prime}, V_{1}\right) .
\end{aligned}
$$

The group $G_{Q}:=\operatorname{GL}\left(V_{1}\right) \times \mathrm{GL}\left(V_{1}^{\prime}\right) \times \mathrm{GL}\left(V_{2}\right)$ naturally acts on $\mathcal{H}$ :

$\left(g_{1}, g_{1}^{\prime}, g_{2}\right)\left(A, A^{\prime}, A^{\prime \prime}, B, B^{\prime}, B^{\prime \prime}\right)=\left(g_{2} A g_{1}^{-1}, g_{2} A^{\prime} g_{1}^{\prime-1}, g_{1}^{\prime} A^{\prime \prime} g_{1}^{-1}, g_{1} B g_{2}^{-1}, g_{1}^{\prime} B^{\prime} g_{2}^{-1}, g_{1} B^{\prime \prime} g_{1}^{\prime-1}\right)$.

The projections $\mathrm{pr}_{12}, \mathrm{pr}_{1^{\prime} 2}, \mathrm{pr}_{11^{\prime}}$ are equivariant with respect to the same named projections from $G_{\mathcal{Q}}$ to $\mathrm{GL}\left(V_{1}\right) \times \mathrm{GL}\left(V_{2}\right), \mathrm{GL}\left(V_{1}^{\prime}\right) \times \mathrm{GL}\left(V_{2}\right), \mathrm{GL}\left(V_{1}\right) \times \mathrm{GL}\left(V_{1}^{\prime}\right)$.

Given $\mathcal{M}_{1^{\prime} 2} \in \operatorname{Coh}^{\mathrm{GL}\left(V_{1}^{\prime}\right) \times \mathrm{GL}\left(V_{2}\right)}\left(\operatorname{Hom}\left(V_{1}^{\prime}, V_{2}\right) \times \operatorname{Hom}\left(V_{2}, V_{1}^{\prime}\right)\right) \quad$ and $\mathcal{M}_{11^{\prime}} \in \operatorname{Coh}^{\mathrm{GL}\left(V_{1}\right) \times \mathrm{GL}\left(V_{1}^{\prime}\right)}\left(\operatorname{Hom}\left(V_{1}, V_{1}^{\prime}\right) \times \operatorname{Hom}\left(V_{1}^{\prime}, V_{1}\right)\right)$ we set

$$
\begin{aligned}
& \mathcal{M}_{11^{\prime}} * \mathcal{M}_{1^{\prime} 2}:=\operatorname{pr}_{12 *}\left(\operatorname{pr}_{11^{\prime}}^{*} \mathcal{M}_{11^{\prime}}\right.\left.\otimes_{\mathbb{C}[\mathcal{H}]} \mathbb{C}\left[Q^{A}\right] \otimes_{\mathbb{C}[\mathcal{H}]} \operatorname{pr}_{1^{\prime} 2}^{*} \mathcal{M}_{1^{\prime} 2}\right)^{\mathrm{GL}\left(V_{1}^{\prime}\right)} \in \\
& \operatorname{Coh}^{\mathrm{GL}\left(V_{1}\right) \times \mathrm{GL}\left(V_{2}\right)}\left(\operatorname{Hom}\left(V_{1}, V_{2}\right) \times \operatorname{Hom}\left(V_{2}, V_{1}\right)\right), \\
& \mathcal{M}_{11^{\prime}} \stackrel{B}{*} \mathcal{M}_{1^{\prime} 2}:=\operatorname{pr}_{12 *}\left(\operatorname{pr}_{11^{\prime}}^{*} \mathcal{M}_{11^{\prime}} \otimes_{\mathbb{C}[\mathcal{H}]} \mathbb{C}\left[Q^{B}\right] \otimes_{\mathbb{C}[\mathcal{H}]} \operatorname{pr}_{1^{\prime} 2}^{*} \mathcal{M}_{1^{\prime} 2}\right)^{\mathrm{GL}\left(V_{1}^{\prime}\right)} \in \\
& \operatorname{Coh}^{\mathrm{GL}\left(V_{1}\right) \times \mathrm{GL}\left(V_{2}\right)}\left(\operatorname{Hom}\left(V_{1}, V_{2}\right) \times \operatorname{Hom}\left(V_{2}, V_{1}\right)\right) .
\end{aligned}
$$

We will actually need the following modifications of these functors:

$\stackrel{B}{*}: D_{\text {perf }}^{G_{\overline{0}}}\left(\mathfrak{G}_{0,2}^{\bullet}\right) \times D_{\text {perf }}^{G_{\overline{0}}}\left(\mathfrak{G}_{0,2}^{\bullet}\right) \rightarrow D_{\text {perf }}^{G_{\overline{0}}}\left(\mathfrak{G}_{0,2}^{\bullet}\right), \stackrel{A}{*}: D_{\text {perf }}^{G_{\overline{0}}}\left(\mathfrak{G}_{2,0}^{\bullet}\right) \times D_{\text {perf }}^{G_{\overline{0}}}\left(\mathfrak{G}_{2,0}^{\bullet}\right) \rightarrow D_{\text {perf }}^{G_{\overline{0}}}\left(\mathfrak{G}_{2,0}^{\bullet}\right)$ obtained using the dg-algebras with trivial differentials $\mathbb{C}[\mathcal{H}]_{0,2}^{\bullet}$ and $\mathbb{C}[\mathcal{H}]_{2,0}^{\bullet}$ respectively, where

$$
\mathbb{C}[\mathcal{H}]_{0,2}^{\bullet}=\operatorname{Sym}\left(\operatorname{Hom}\left(V_{1}, V_{2}\right)\right) \otimes \operatorname{Sym}\left(\operatorname{Hom}\left(V_{2}, V_{1}\right)[-2]\right) \otimes \operatorname{Sym}\left(\operatorname{Hom}\left(V_{1}^{\prime}, V_{2}\right)\right)
$$

$\otimes \operatorname{Sym}\left(\operatorname{Hom}\left(V_{2}, V_{1}^{\prime}\right)[-2]\right) \otimes \operatorname{Sym}\left(\operatorname{Hom}\left(V_{1}, V_{1}^{\prime}\right)\right) \otimes \operatorname{Sym}\left(\operatorname{Hom}\left(V_{1}^{\prime}, V_{1}\right)[-2]\right)$, 


$$
\begin{aligned}
\mathbb{C}[\mathcal{H}]_{2,0}^{\bullet} & =\operatorname{Sym}\left(\operatorname{Hom}\left(V_{1}, V_{2}\right)[-2]\right) \otimes \operatorname{Sym}\left(\operatorname{Hom}\left(V_{2}, V_{1}\right)\right) \otimes \operatorname{Sym}\left(\operatorname{Hom}\left(V_{1}^{\prime}, V_{2}\right)[-2]\right) \\
& \otimes \operatorname{Sym}\left(\operatorname{Hom}\left(V_{2}, V_{1}^{\prime}\right)\right) \otimes \operatorname{Sym}\left(\operatorname{Hom}\left(V_{1}, V_{1}^{\prime}\right)[-2]\right) \otimes \operatorname{Sym}\left(\operatorname{Hom}\left(V_{1}^{\prime}, V_{1}\right)\right) .
\end{aligned}
$$

(we identify $\mathbb{C}[\operatorname{Hom}(U, W)]$ with $\operatorname{Sym} \operatorname{Hom}(W, U)$ ).

\subsection{Localization, coherent. We identify}

$$
\operatorname{Hom}\left(V_{1}, V_{2}\right)=\operatorname{Hom}\left(V_{2}, V_{1}\right)^{*}, \operatorname{Hom}\left(V_{2}, V_{1}\right)=\operatorname{Hom}\left(V_{1}, V_{2}\right)^{*},
$$

so that

$$
\operatorname{Sym}\left(\operatorname{Hom}\left(V_{1}, V_{2}\right)\right)=\mathbb{C}\left[\operatorname{Hom}\left(V_{2}, V_{1}\right)\right], \operatorname{Sym}\left(\operatorname{Hom}\left(V_{2}, V_{1}\right)\right)=\mathbb{C}\left[\operatorname{Hom}\left(V_{1}, V_{2}\right)\right] .
$$

We have an open subvariety $\operatorname{Isom}\left(V_{2}, V_{1}\right) \subset \operatorname{Hom}\left(V_{2}, V_{1}\right)$, so that $\mathbb{C}\left[\operatorname{Hom}\left(V_{2}, V_{1}\right)\right] \subset \mathbb{C}\left[\operatorname{Isom}\left(V_{2}, V_{1}\right)\right]$. We set

$$
\mathfrak{B}^{\bullet}:=\mathbb{C}\left[\operatorname{Isom}\left(V_{2}, V_{1}\right)\right] \otimes \operatorname{Sym}\left(\operatorname{Hom}\left(V_{2}, V_{1}\right)[-2]\right)
$$

(a dg-algebra with trivial differential). Similarly, we define

$$
\mathfrak{A}^{\bullet}:=\operatorname{Sym}\left(\operatorname{Hom}\left(V_{1}, V_{2}\right)[-2]\right) \otimes \mathbb{C}\left[\operatorname{Isom}\left(V_{1}, V_{2}\right)\right]
$$

An equivalent formulation of [BF, Theorem 5] is an existence of a monoidal equivalence $D_{\text {perf }}^{G_{\overline{0}}}\left(\mathfrak{A}^{\bullet}\right) \cong D_{\mathbf{G}_{\mathbf{O}}}(\mathbf{G r})$ (and, changing the roles of $V_{1}, V_{2}$, a monoidal equivalence $\left.D_{\text {perf }}^{G_{\overline{0}}}\left(\mathfrak{B}^{\bullet}\right) \cong D_{\mathbf{G}_{\mathbf{O}}}(\mathbf{G r})\right)$.

Since $\mathfrak{A}^{\bullet}$ (resp. $\mathfrak{B}^{\bullet}$ ) is a localization of $\mathfrak{G}_{2,0}^{\bullet}$ (resp. of $\mathfrak{G}_{0,2}^{\bullet}$ ), we have the restriction of scalars functors

$$
\operatorname{Res}_{A}: D_{\text {perf }}^{G_{\overline{0}}}\left(\mathfrak{A}^{\bullet}\right) \rightarrow \hat{D}_{\text {perf }}^{G_{\overline{0}}}\left(\mathfrak{G}_{2,0}^{\bullet}\right), \operatorname{Res}_{B}: D_{\text {perf }}^{G_{\overline{0}}}\left(\mathfrak{B}^{\bullet}\right) \rightarrow \hat{D}_{\text {perf }}^{G_{\overline{0}}}\left(\mathfrak{G}_{0,2}^{\bullet}\right),
$$

where $\hat{D}_{\text {perf }}^{G_{\overline{0}}}\left(\mathfrak{G}_{?, ?}^{\bullet}\right)$ stands for the Ind-completion of $D_{\text {perf }}^{G_{\overline{0}}}\left(\mathfrak{G}_{?, ?}^{\bullet}\right)$.

However, one can check that for $\mathcal{N} \in D_{\text {perf }}^{G_{\overline{0}}}\left(\mathfrak{A}^{\bullet}\right)$ and $\mathcal{M} \in D_{\text {perf }}^{G_{\overline{0}}}\left(\mathfrak{G}_{2,0}^{\bullet}\right)$ both convolutions $\operatorname{Res}_{A}(\mathcal{N}) \stackrel{A}{*} \mathcal{M}$ and $\mathcal{M}{ }^{A} * \operatorname{Res}_{A}(\mathcal{N})$ lie in $D_{\text {perf }}^{G_{\overline{0}}}\left(\mathfrak{G}_{2,0}^{\bullet}\right) \subset \hat{D}_{\text {perf }}^{G_{\overline{0}}}\left(\mathfrak{G}_{2,0}^{\bullet}\right)$. Thus we have the left and right convolution actions

$\stackrel{A}{*}: D_{\text {perf }}^{G_{\overline{0}}}\left(\mathfrak{A}^{\bullet}\right) \times D_{\text {perf }}^{G_{\overline{0}}}\left(\mathfrak{G}_{2,0}^{\bullet}\right) \rightarrow D_{\text {perf }}^{G_{\overline{0}}}\left(\mathfrak{G}_{2,0}^{\bullet}\right), D_{\text {perf }}^{G_{\overline{0}}}\left(\mathfrak{G}_{2,0}^{\bullet}\right) \times D_{\text {perf }}^{G_{\overline{0}}}\left(\mathfrak{A}^{\bullet}\right) \rightarrow D_{\text {perf }}^{G_{\overline{0}}}\left(\mathfrak{G}_{2,0}^{\bullet}\right)$, and similarly

$\stackrel{B}{*}: D_{\text {perf }}^{G_{\overline{0}}}\left(\mathfrak{B}^{\bullet}\right) \times D_{\text {perf }}^{G_{\overline{0}}}\left(\mathfrak{G}_{0,2}^{\bullet}\right) \rightarrow D_{\text {perf }}^{G_{\overline{0}}}\left(\mathfrak{G}_{0,2}^{\bullet}\right), D_{\text {perf }}^{G_{\overline{0}}}\left(\mathfrak{G}_{0,2}^{\bullet}\right) \times D_{\text {perf }}^{G_{\overline{0}}}\left(\mathfrak{B}^{\bullet}\right) \rightarrow D_{\text {perf }}^{G_{\overline{0}}}\left(\mathfrak{G}_{0,2}^{\bullet}\right)$.

3.6. Renormalizations. The action of the centre $Z\left(\mathrm{GL}\left(V_{1}\right)\right) \cong \mathbb{G}_{m}$ on an object $\mathcal{M} \in D_{\text {perf }}^{G_{\overline{0}}}\left(\mathfrak{G}_{1,1}^{\bullet}\right)$ defines a grading, and the corresponding degrees will be denoted by $\operatorname{deg}_{1}$. Similarly, the action of $Z\left(\mathrm{GL}\left(V_{2}\right)\right) \cong \mathbb{G}_{m}$ gives rise to another grading with degrees denoted by $\operatorname{deg}_{2}$. The cohomological degrees will be denoted simply by deg. Clearly, the degrees of the generators are as follows:

$$
\operatorname{deg}_{2}\left(\operatorname{Hom}\left(V_{1}, V_{2}\right)\right)=\operatorname{deg}_{1}\left(\operatorname{Hom}\left(V_{2}, V_{1}\right)\right)=1
$$




$$
\operatorname{deg}_{1}\left(\operatorname{Hom}\left(V_{1}, V_{2}\right)\right)=\operatorname{deg}_{2}\left(\operatorname{Hom}\left(V_{1}, V_{2}\right)\right)=-1 .
$$

Hence changing cohomological degrees by the formula deg $\rightsquigarrow \operatorname{deg}+\operatorname{deg}_{1}$, resp. $\operatorname{deg} \rightsquigarrow \operatorname{deg}-\operatorname{deg}_{2}$, yields equivalences

$$
\begin{gathered}
D_{\text {perf }}^{G_{\overline{0}}}\left(\mathfrak{G}_{2,0}^{\bullet}\right) \stackrel{\varrho_{\text {right }}}{\longrightarrow} D_{\text {perf }}^{G_{\overline{0}}}\left(\mathfrak{G}_{1,1}^{\bullet}\right) \stackrel{\varrho_{\text {right }}}{\longrightarrow} D_{\text {perf }}^{G_{\overline{0}}}\left(\mathfrak{G}_{0,2}^{\bullet}\right), \\
D_{\text {perf }}^{G_{\overline{0}}}\left(\mathfrak{G}_{2,0}^{\bullet}\right) \stackrel{\varrho_{\text {left }}}{\longrightarrow} D_{\text {perf }}^{G_{\overline{0}}}\left(\mathfrak{G}_{1,1}^{\bullet}\right) \stackrel{\varrho_{\text {left }}}{\longrightarrow} D_{\text {perf }}^{G_{\overline{0}}}\left(\mathfrak{G}_{0,2}^{\bullet}\right),
\end{gathered}
$$

respectively. The notation is due to the fact that $\varrho_{\text {left }}$ commutes with the left action of $\operatorname{Rep}\left(\mathrm{GL}\left(V_{1}\right)\right)$ on our categories, while $\varrho_{\text {right }}$ commutes with the right action of $\operatorname{Rep}\left(\mathrm{GL}\left(V_{2}\right)\right)$ on our categories.

Now recall the notation in the definition of categories $D_{? \mathbf{G}_{\mathbf{O}}}(\mathbf{G r} \times \mathbf{V})$ (where $?=!, *, ! *)$ of Section 3.1. Given $\mathcal{F}=\left(\mathcal{F}_{n}\right)_{n>m} \in D_{!_{\mathbf{G}}}(\mathbf{G r} \times \mathbf{V})$ we define $\varrho_{\text {right }} \mathcal{F}:=\left(\mathcal{F}_{n}[-N n]\right)_{n>m} \in D_{! * \mathbf{G}_{\mathbf{O}}}(\mathbf{G r} \times \mathbf{V})$. Similarly, given $\mathcal{F}=\left(\mathcal{F}_{n}\right)_{n>m} \in$ $D_{! * \mathbf{G}_{\mathbf{O}}}(\mathbf{G r} \times \mathbf{V})$ we define $\varrho_{\text {right }} \mathcal{F}:=\left(\mathcal{F}_{n}[-N n]\right)_{n>m} \in D_{* \mathbf{G}_{\mathbf{O}}}(\mathbf{G r} \times \mathbf{V})$. The functors $\varrho_{\text {right }}$ commute with the action of the monoidal category $D_{\mathbf{G}_{\mathbf{O}}}(\mathbf{G r})$ by the right convolutions. Recall also that the affine Grassmannian is a union of connected components $\mathbf{G r}=\bigsqcup_{k \in \mathbb{Z}} \mathbf{G r}^{(k)}$, where $\mathbf{G r}^{(k)}$ parametrizes the lattices of virtual dimension $k$ (e.g. $\left.\operatorname{dim}\left(t \mathbf{V}_{0}\right)=-N\right)$. For $\mathcal{F}$ supported on $\mathbf{G r}^{(k)} \times \mathbf{V}$ we set $\varrho_{\text {left }}(\mathcal{F}):=\varrho_{\text {right }}(\mathcal{F})[-k]$. Then the functors

$$
\varrho_{\text {left }}: D_{! \mathbf{G}_{\mathbf{O}}}(\mathbf{G r} \times \mathbf{V}) \rightarrow D_{! * \mathbf{G}_{\mathbf{O}}}(\mathbf{G r} \times \mathbf{V}), D_{! * \mathbf{G}_{\mathbf{O}}}(\mathbf{G r} \times \mathbf{V}) \rightarrow D_{* \mathbf{G}_{\mathbf{O}}}(\mathbf{G r} \times \mathbf{V})
$$

commute with the action of the monoidal category $D_{\mathbf{G}_{\mathbf{O}}}(\mathbf{G r})$ by the left convolutions.

Our goal is the following

Theorem 3.6.1. There exist monoidal ${ }^{7}$ equivalences of triangulated categories

$$
\begin{aligned}
& \left(D_{\text {perf }}^{G_{\overline{0}}}\left(\mathfrak{G}_{2,0}^{\bullet}\right), \quad \stackrel{A}{*}\right) \underset{\Phi^{2,0}}{\stackrel{\sim}{\longrightarrow}}\left(D ! \mathbf{G}_{\mathbf{O}}(\mathbf{G r} \times \mathbf{V}), \stackrel{!}{\circledast}\right)
\end{aligned}
$$

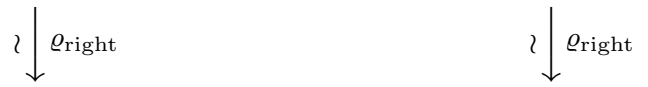

$$
\begin{aligned}
& \left(D_{\text {perf }}^{G_{\overline{0}}}\left(\mathfrak{G}_{1,1}^{\bullet}\right), \otimes_{\mathfrak{G}_{1,1}}\right) \underset{\Phi^{1,1}}{\stackrel{\sim}{\longrightarrow}}\left(D_{! * \mathbf{G}_{\mathbf{O}}}(\mathbf{G r} \times \mathbf{V}), \star\right)
\end{aligned}
$$

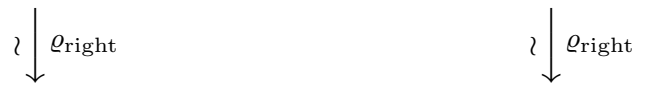

$$
\begin{aligned}
& \left(D_{\text {perf }}^{G_{\overline{0}}}\left(\mathfrak{G}_{0,2}^{\bullet}\right), \stackrel{B}{*}\right) \underset{\Phi^{0,2}}{\stackrel{\sim}{\longrightarrow}}\left(D_{* \mathbf{G}_{\mathbf{O}}}(\mathbf{G r} \times \mathbf{V}), \stackrel{*}{*}\right) .
\end{aligned}
$$

(the vertical equivalences are not monoidal). The squares are commutative. The horizontal equivalences commute with the actions of the monoidal spherical Hecke category $\operatorname{Perv}_{\mathbf{G}_{\mathbf{O}}}(\mathbf{G r}) \cong \operatorname{Rep}\left(\mathrm{GL}_{N}\right)$ by the left and right convolutions.

The proof will be given in Section 3.16 after a necessary preparation.

\footnotetext{
${ }^{7}$ see Section 3.7 for the definition of the left middle monoidal structure.
} 
3.7. Super. Strictly speaking, in accordance with the footnote at the beginning of Section 3.4, we should consider the category $S D_{\text {perf }}^{G_{\overline{0}}}\left(\mathfrak{G}_{1,1}^{\bullet}\right)$ of super dg-modules over the superalgebra $\mathfrak{G}_{1,1}^{\bullet}=\operatorname{Sym}\left(\mathfrak{g}_{1}[-1]\right)$. The latter superalgebra is supercommutative, hence we have a symmetric monoidal structure $\otimes_{\mathfrak{G}_{1,1}}$ on the category $S D_{\text {perf }}^{G_{\overline{0}}}\left(\mathfrak{G}_{1,1}^{\bullet}\right)$. The equivalence $\Phi^{1,1}$ of Theorem 3.6.1 can be upgraded to a monoidal equivalence $S \Phi^{1,1}:\left(S D_{\text {perf }}^{G_{\overline{0}}}\left(\mathfrak{G}_{1,1}^{\bullet}\right), \otimes_{\mathfrak{G}_{1,1}}\right) \stackrel{\sim}{\longrightarrow}\left(S D_{! * \mathbf{G}_{\mathbf{O}}}(\mathbf{G r} \times \mathbf{V}), \star\right)$ to the derived category of sheaves with coefficients in super vector spaces.

However, the action of the central element $\left(\operatorname{Id}_{V_{1}},-\mathrm{Id}_{V_{2}}\right) \in G_{\overline{0}}$ on an object of $D_{\text {perf }}^{G_{\overline{0}}}\left(\mathfrak{G}_{1,1}^{\bullet}\right)$ equips this object with an extra $\mathbb{Z} / 2 \mathbb{Z}$-grading, and thus defines a fully faithful functor $D_{\text {perf }}^{G_{\overline{0}}}\left(\mathfrak{G}_{1,1}^{\bullet}\right) \rightarrow S D_{\text {perf }}^{G_{\overline{0}}}\left(\mathfrak{G}_{1,1}^{\bullet}\right)$ of a "superization", such that its essential image is closed under the monoidal structure $\otimes_{\mathfrak{G}_{1,1}}$. This defines the desired monoidal structure $\otimes_{\mathfrak{G}_{1,1}}$ on the category $D_{\text {perf }}^{G_{\overline{0}}}\left(\mathfrak{G}_{1,1}^{\bullet}\right)$.

3.8. Koszul equivalence. We consider the following complex $H^{\bullet}$ of odd vector spaces living in degrees $0,1: \mathfrak{g}_{\overline{1}} \stackrel{\mathrm{Id}}{\longrightarrow} \mathfrak{g}_{\overline{1}}$. We define the Koszul complex $K^{\bullet}$ as the $\operatorname{symmetric~algebra~} \operatorname{Sym}\left(H^{\bullet}\right)$. The degree zero part

$$
K^{0}=\Lambda\left(\operatorname{Hom}\left(V_{1}, V_{2}\right) \oplus \operatorname{Hom}\left(V_{2}, V_{1}\right)\right)=: \Lambda
$$

(as a vector space, with a super-structure disregarded). We turn $K^{\bullet}$ into a dg- $\mathfrak{G}_{1,1}^{\bullet}-\Lambda$-bimodule by letting $\mathfrak{G}_{1,1}^{\bullet}$ act by multiplication, and $\Lambda$ by differentiation. Note that $K^{\bullet}$ is quasi-isomorphic to $\mathbb{C}$ in degree 0 as a complex of vector spaces, but not as a dg- $\mathfrak{G}_{1,1}^{\bullet}-\Lambda$-bimodule. We consider the derived category $D_{\mathrm{fd}}^{G_{\overline{0}}}(\Lambda)$ of finite dimensional complexes of $G_{\overline{0}} \ltimes \Lambda$-modules. If we remember the super-structure of $\Lambda$, we obtain the corresponding category of super dg-modules $S D_{\mathrm{fd}}^{G_{\overline{0}}}(\Lambda)$. We have the Koszul equivalence functors

$$
\varkappa: D_{\mathrm{fd}}^{G_{\overline{0}}}(\Lambda) \stackrel{\sim}{\longrightarrow} D_{\text {perf }}^{G_{\overline{0}}}\left(\mathfrak{G}_{1,1}^{\bullet}\right), S D_{\mathrm{fd}}^{G_{\overline{0}}}(\Lambda) \stackrel{\sim}{\longrightarrow} S D_{\text {perf }}^{G_{\overline{0}}}\left(\mathfrak{G}_{1,1}^{\bullet}\right), \mathcal{N} \mapsto K^{\bullet} \otimes_{\Lambda} \mathcal{N} .
$$

Here is an equivalent definition of the category $S D_{\mathrm{fd}}^{G_{\overline{0}}}(\Lambda)$. We consider the following degeneration $\mathfrak{g l}(N \mid N)$ of the Lie superalgebra $\mathfrak{g l}(N \mid N)$ : the supercommutator of the even elements (with even or odd elements) remains intact, but the supercommutator of any two odd elements is set to be zero. Let $S D_{\text {int }}(\mathfrak{g l}(N \mid N))$ denote the derived category of bounded complexes of integrable $\mathfrak{g l}(N \mid N)$-modules (note that the even part of $\underline{\mathfrak{g l}}(N \mid N)$ is just $\mathfrak{g}_{\overline{0}}$, and the integrability is nothing but $\mathfrak{g}_{\overline{0}}$-integrability, i.e. $G_{\overline{0}}$-equivariance). Then $S D_{\text {int }}(\underline{\mathfrak{g l}}(N \mid N)) \cong S D_{\mathrm{fd}}^{G_{\overline{0}}}(\Lambda)$ tautologically. The resulting Koszul equivalence $\varkappa: S D_{\text {int }}(\underline{\mathfrak{g l}}(N \mid N)) \stackrel{\sim}{\longrightarrow} S D_{\text {perf }}^{G_{\overline{0}}}\left(\mathfrak{G}_{1,1}^{\bullet}\right)$ is monoidal with respect to the usual tensor structure on the LHS and $\otimes_{\mathfrak{G}_{i, 1}}$ on the RHS.

As in Section 3.7, the action of $\left(\operatorname{Id}_{V_{1}},-\mathrm{Id}_{V_{2}}\right) \in G_{\overline{0}}$ gives rise to a "superization" fully faithful functor $D_{\mathrm{fd}}^{G_{\overline{0}}}(\Lambda) \rightarrow S D_{\mathrm{fd}}^{G_{\overline{0}}}(\Lambda) \cong S D_{\text {int }}(\underline{\mathfrak{g l}}(N \mid N))$ with the essential 
image closed under the tensor structure. This defines the tensor structure on $D_{\mathrm{fd}}^{G_{\overline{0}}}(\Lambda)$ such that the Koszul equivalence $\varkappa: D_{\mathrm{fd}}^{G_{\overline{0}}}(\Lambda) \stackrel{\sim}{\longrightarrow} D_{\text {perf }}^{G_{\overline{0}}}\left(\mathfrak{G}_{1,1}^{\bullet}\right)$ is monoidal.

Corollary 3.8.1 (of Theorem 3.6.1). (a) The composed equivalence

$$
\Phi^{1,1} \circ \varkappa: D_{\mathrm{fd}}^{G_{\overline{0}}}(\Lambda) \stackrel{\sim}{\longrightarrow} D_{! * \mathbf{G}_{\mathbf{O}}}(\mathbf{G r} \times \mathbf{V})
$$

is exact with respect to the tautological t-structure on $D_{\mathrm{fd}}^{G_{\overline{0}}}(\Lambda)$ and the perverse t-structure on $D_{! * \mathbf{G}_{\mathbf{O}}}(\mathbf{G r} \times \mathbf{V})$.

(b) This equivalence is monoidal with respect to the tensor structure on $D_{\mathrm{fd}}^{G_{\overline{0}}}(\Lambda)$ and the fusion $\star$ on $D_{! * \mathbf{G}_{\mathbf{O}}}(\mathbf{G r} \times \mathbf{V})$.

(c) The equivariant derived category $D_{! * \mathbf{G}_{\mathbf{O}}}(\mathbf{G r} \times \mathbf{V})$ is equivalent to the bounded derived category of the abelian category $\operatorname{Perv}_{\mathbf{G}_{\mathbf{O}}}(\mathbf{G r} \times \mathbf{V})$.

Proof. We consider an irreducible $G_{\overline{0}}$-module $V_{1, \boldsymbol{\lambda}} \otimes V_{2, \boldsymbol{\mu}}$ as a $G_{\overline{0}} \ltimes \Lambda$-module with the trivial action of $\Lambda$. Then $\varkappa\left(V_{1, \boldsymbol{\lambda}} \otimes V_{2, \boldsymbol{\mu}}\right)=V_{1, \boldsymbol{\lambda}} \otimes \mathfrak{G}_{1,1}^{\bullet} \otimes V_{2, \boldsymbol{\mu}}$, and $\Phi^{1,1}\left(V_{1, \boldsymbol{\lambda}} \otimes \mathfrak{G}_{1,1}^{\bullet} \otimes V_{2, \boldsymbol{\mu}}\right)=\mathrm{IC}_{\left(\boldsymbol{\lambda}^{*}, \boldsymbol{\mu}^{*}\right)}$ by construction of $\Phi^{1,1}$. Here for a signature $\boldsymbol{\nu}=\left(\nu_{1} \geq \ldots \geq \nu_{N}\right)$ we set $\boldsymbol{\nu}^{*}:=\left(-\nu_{N} \geq \ldots \geq-\nu_{1}\right)$.

Finally, since $D_{\mathrm{fd}}^{G_{\overline{0}}}(\Lambda)$ is equivalent to the bounded derived category of its heart $\operatorname{Rep}\left(G_{\overline{0}} \ltimes \Lambda\right)$, (c) follows from (a).

3.9. Deequivariantized Ext algebra. Recall from [FGT, Proposition 8] that the $\mathbf{G}_{\mathbf{O}^{-} \text {orbits in }} \mathbf{G r} \times \stackrel{\circ}{\mathbf{V}}$ are numbered by bisignatures $(\boldsymbol{\lambda}, \boldsymbol{\mu})$ where both $\boldsymbol{\lambda}$ and $\boldsymbol{\mu}$ have length $N$. The IC-extension of the constant 1-dimensional local system on such an orbit is denoted by $\mathrm{IC}_{(\boldsymbol{\lambda}, \boldsymbol{\mu})}$. In particular, $\mathrm{IC}_{\left(0^{N}, 0^{N}\right)}$ is the constant sheaf on $\mathbf{G r}^{0} \times \mathbf{V}_{0}$, to be denoted by $E_{0}$ for short. Also recall from [FGT, Section 3] that the left and right actions of the monoidal Satake category $\operatorname{Perv}_{\mathbf{G}_{\mathbf{O}}}(\mathbf{G r}) \cong \operatorname{Rep}\left(\mathrm{GL}_{N}\right)$ on $D_{! * \mathbf{G}_{\mathbf{O}}}(\mathbf{G r} \times \mathbf{V})$ by convolutions respect the perverse $t$-structure with the heart $\operatorname{Perv}_{\mathbf{G}_{\mathbf{O}}}(\mathbf{G r} \times \mathbf{V}) \subset D_{! * \mathbf{G}_{\mathbf{O}}}(\mathbf{G r} \times \mathbf{V})$. As has been mentioned in Section 3.6, the right actions of $D_{\mathbf{G}_{\mathbf{O}}}(\mathbf{G r})$ on $D_{? \mathbf{G}_{\mathbf{O}}}(\mathbf{G r} \times \mathbf{V})$ commute with the equivalences $\varrho_{\text {right }}$, but the left actions only commute with $\varrho_{\text {right }}$ up to cohomological shifts depending on the connected components of Gr.

We restrict the left and right actions of $D_{\mathbf{G}_{\mathbf{O}}}(\mathbf{G r})$ on $D_{? \mathbf{G}_{\mathbf{O}}}(\mathbf{G r} \times \mathbf{V})$ to the left and right actions of $\operatorname{Perv}_{\mathbf{G}_{\mathbf{O}}}(\mathbf{G r}) \cong \operatorname{Rep}\left(\mathrm{GL}_{N}\right)$. Thus we obtain the action of $\operatorname{Rep}(\mathrm{G})$ for $\mathrm{G}=\mathrm{GL}_{N} \times \mathrm{GL}_{N}$. Let $D_{? \mathbf{G}_{\mathbf{O}}}^{\text {deeq }}(\mathbf{G r} \times \mathbf{V})$ denote the corresponding deequivariantized category (see [AG2] in the setting of abelian categories and [G2] in the setting of dg-categories). Recall that to construct $D_{? \mathbf{G}_{\mathbf{O}}}^{\text {deeq }}(\mathbf{G r} \times \mathbf{V})$, first we consider objects of the ind-completion of $D_{? \mathbf{G}_{\mathbf{O}}}(\mathbf{G r} \times \mathbf{V})$ endowed with an action of $R_{\mathrm{G}}$ where $R_{\mathrm{G}}$ is the regular representation of $\mathrm{G}$ considered as a ring-object in $\operatorname{Rep}(\mathrm{G})$. Then we take compact objects of the resulting category. More explicitly, an object of $D_{? \mathbf{G}_{\mathbf{O}}}^{\text {deeq }}(\mathbf{G r} \times \mathbf{V})$ is an object $\mathcal{F}$ of $D_{? \mathbf{G}_{\mathbf{O}}}(\mathbf{G r} \times \mathbf{V})$ together with a system of isomorphisms $\mathrm{IC}_{\boldsymbol{\lambda}} * \mathcal{F} * \mathrm{IC}_{\boldsymbol{\mu}} \stackrel{\sim}{\longrightarrow} V_{\boldsymbol{\lambda}}^{*} \otimes V_{\boldsymbol{\mu}}^{*} \otimes \mathcal{F}$ (recall that the geometric Satake 
equivalence takes $\mathrm{IC}_{\boldsymbol{\lambda}}$ to $V_{\boldsymbol{\lambda}}^{*}$ ) for any signatures $\boldsymbol{\lambda}, \boldsymbol{\mu}$, satisfying some natural compatibilities with respect to direct sums and tensor products. By definition we have a natural forgetful functor $D_{? \mathbf{G}_{\mathbf{O}}}^{\text {deeq }}(\mathbf{G r} \times \mathbf{V}) \rightarrow D_{? \mathbf{G}_{\mathbf{O}}}(\mathbf{G r} \times \mathbf{V})$. This functor admits a left adjoint that sends an object $\mathcal{F}$ to $R_{\mathrm{G}} * \mathcal{F}=\bigoplus_{\boldsymbol{\lambda}, \boldsymbol{\mu}} V_{\boldsymbol{\lambda}} \otimes V_{\boldsymbol{\mu}} \otimes \mathrm{IC}_{\boldsymbol{\lambda}} * \mathcal{F} * \mathrm{IC}_{\boldsymbol{\mu}}$.

Thus, given $\mathcal{F}_{1}, \mathcal{F}_{2} \in D_{\text {? }} \mathbf{\mathbf { G } _ { \mathbf { O } }}(\mathbf{G r} \times \mathbf{V})$, we denote the corresponding objects of the deequivariantized category by the same symbols, and we have

$\operatorname{RHom}_{D_{? \mathbf{G}_{\mathbf{O}}}^{\text {deeq }}(\mathbf{G r} \times \mathbf{V})}\left(\mathcal{F}_{1}, \mathcal{F}_{2}\right)=\bigoplus_{\boldsymbol{\lambda}, \boldsymbol{\mu}} \operatorname{RHom}_{D_{? \mathbf{G}_{\mathbf{O}}}}(\mathbf{G r} \times \mathbf{V})\left(\mathcal{F}_{1}, \mathrm{IC}_{\boldsymbol{\lambda}} * \mathcal{F}_{2} * \mathrm{IC}_{\boldsymbol{\mu}}\right) \otimes V_{\boldsymbol{\lambda}} \otimes V_{\boldsymbol{\mu}}$.

Lemma 3.9.1. The dg-algebra $\mathrm{RHom}_{D_{! * \mathrm{G}_{\mathbf{O}}}^{\text {deeq }}(\mathbf{G r} \times \mathbf{V})}\left(E_{0}, E_{0}\right)$ is formal, i.e. it is quasiisomorphic to the graded algebra $\mathrm{Ext}_{D_{! * \mathrm{G}_{\mathbf{O}}}^{\bullet}(\mathbf{G r} \times \mathbf{V})}\left(E_{0}, E_{0}\right)$ with trivial differential.

Proof. We change the setting to the base field $\mathbb{F}_{q}$. Then a $\mathbf{G}_{\mathbf{O}^{-} \text {-equivariant }}$ irreducible perverse sheaf $\mathrm{IC}_{(\boldsymbol{\lambda}, \boldsymbol{\mu})}$ on $\mathbf{G r} \times \mathbf{V}$ carries a natural Weil structure, see [FGT, Section 2.6], and $\operatorname{Ext}_{D_{! * \mathbf{G}_{\mathbf{O}}}(\mathbf{G r} \times \mathbf{V})}\left(E_{0}, \mathrm{IC}_{(\boldsymbol{\lambda}, \boldsymbol{\mu})}\right)$ is pure. Indeed, if $\bar{\imath}_{0}$ denotes the closed embedding $\mathbf{V}_{0} \cong \mathbf{G r}^{0} \times \mathbf{V}_{0} \hookrightarrow \mathbf{G r} \times \mathbf{V}$, then $\operatorname{Ext}_{D_{! * \mathbf{G}_{\mathbf{O}}}(\mathbf{G r} \times \mathbf{V})}\left(E_{0}, \mathrm{IC}_{(\boldsymbol{\lambda}, \boldsymbol{\mu})}\right)=H_{\mathbf{G}_{\mathbf{O}}}^{\bullet}\left(\mathbf{V}_{0}, \bar{\imath}_{0}^{!} \mathrm{IC}_{(\boldsymbol{\lambda}, \boldsymbol{\mu})}\right)$. Let us consider the loop rotation action $\mathbb{G}_{m} \curvearrowright(\mathbf{G r} \times \mathbf{V})$. We have an embedding $\mathbb{A}^{1} \hookrightarrow \mathbf{V}$ (constant Laurent series), and $\mathbf{G r}^{0} \times \mathbb{A}^{1}$ is a fixed point component of the $\mathbb{G}_{m}$-action, so that $H_{\mathbf{G}_{\mathbf{O}}}^{\bullet}\left(\mathbf{V}_{0}, \bar{l}_{0} \mathrm{IC}_{(\boldsymbol{\lambda}, \boldsymbol{\mu})}\right)$ is the hyperbolic restriction to this component (more precisely, the hyperbolic restriction is a geometrically constant complex on $\mathbb{A}^{1}$ with the above stalks) [Bra, DG]. But the hyperbolic restriction preserves purity.

Now given the purity of $\operatorname{Ext}_{D_{! * \mathrm{G}_{\mathbf{O}}}(\mathbf{G r} \times \mathbf{V})}\left(E_{0}, \mathrm{IC}_{(\boldsymbol{\lambda}, \boldsymbol{\mu})}\right)$, the desired result follows by an application of [BF, Lemma 15] (and then of [BF, Proposition 5]).

We denote the dg-algebra $\operatorname{Ext}_{D_{! * G_{\mathbf{O}}}^{\bullet \text { deeq }}(\mathbf{G r} \times \mathbf{V})}^{\bullet}\left(E_{0}, E_{0}\right)$ (with trivial differential) by $\mathfrak{E} \bullet$ Since it is an Ext-algebra in the deequivariantized category between objects induced from the original category, it is automatically equipped with an action of $\mathrm{GL}_{N} \times \mathrm{GL}_{N}=\mathrm{GL}\left(V_{1}\right) \times \mathrm{GL}\left(V_{2}\right)=G_{\overline{0}}$, and we can consider the corresponding triangulated category $D_{\text {perf }}^{G_{\overline{0}}}\left(\mathfrak{E}^{\bullet}\right)$.

Lemma 3.9.2. There is a canonical equivalence $D_{\text {perf }}^{G_{\overline{0}}}\left(\mathfrak{E}^{\bullet}\right) \stackrel{\sim}{\longrightarrow} D_{! * \mathbf{G}_{\mathbf{O}}}(\mathbf{G r} \times \mathbf{V})$.

Proof. The desired functor is constructed as in [BF, Section 6.5, Propositions 5,6]. Since $E_{0}$ generates the triangulated category $D_{! * \mathbf{G}_{\mathbf{O}}}(\mathbf{G r} \times \mathbf{V})$ (with respect to the left and right actions of the Satake category), the claim follows from Lemma 3.9.1.

We also consider the left and right actions of the monoidal Satake category $\operatorname{Perv}_{\mathbf{G}_{\mathbf{O}}}(\mathbf{G r}) \cong \operatorname{Rep}\left(\mathrm{GL}_{N}\right)$ on $D_{\mathbf{G}_{\mathbf{O}}}(\mathbf{G r})$ by convolutions. Let $D_{\mathbf{G}_{\mathbf{O}}}^{\text {deq }}(\mathbf{G r})$ 
denote the corresponding deequivariantized category. Then the dg-algebra $\mathrm{RHom}_{D_{\mathbf{G}_{\mathbf{O}}}^{\text {deeq }}(\mathbf{G r})}\left(\mathrm{IC}_{0}, \mathrm{IC}_{0}\right)$ is formal, i.e. it is quasiisomorphic to the graded algebra Ext $_{D_{\mathbf{G}_{\mathbf{O}}}^{\bullet}(\mathbf{G r})}^{\text {deq }}\left(\mathrm{IC}_{0}, \mathrm{IC}_{0}\right)$ with trivial differential. Furthermore, it follows from $[\mathrm{BF}$, Theorem 5] that there is a natural isomorphism $\operatorname{Ext}_{D_{\mathbf{G}_{\mathbf{O}}}^{\bullet}(\mathbf{G r})}\left(\mathrm{IC}_{0}, \mathrm{IC}_{0}\right) \cong \mathfrak{A}^{\bullet}$ (notation of Section 3.5).

3.10. Localization, constructible. We have an automorphism $\alpha: \mathbf{G r} \times \mathbf{V} \stackrel{\sim}{\sim} \mathbf{G r} \times \mathbf{V},(L, v) \mapsto(L, t v)$. We have a morphism of endofunctors $\alpha^{*} \rightarrow \mathrm{Id}: D_{* \mathbf{G}_{\mathbf{O}}}(\mathbf{G r} \times \mathbf{V}) \rightarrow D_{* \mathbf{G}_{\mathbf{O}}}(\mathbf{G r} \times \mathbf{V})$ constructed as follows. We consider a family of automorphisms $\boldsymbol{\alpha}: \mathbb{A}^{1} \times \mathbf{G r} \times \mathbf{V} \hookrightarrow \mathbf{G r} \times \mathbf{V},(c, L, v) \mapsto(L,(c+t) v)$, so that $\boldsymbol{\alpha}_{0}=\alpha$. Note that $\boldsymbol{\alpha}_{c}^{*} \cong \operatorname{Id}$ on $D_{* \mathbf{G}_{\mathbf{O}}}(\mathbf{G r} \times \mathbf{V})$ for $c \neq 0$. Now the desired morphism $\alpha^{*} \rightarrow$ Id is just the cospecialization ${ }^{8}$ morphism from the stalk at $0 \in \mathbb{A}^{1}$ to the nearby stalk. For $\mathcal{F}_{1}, \mathcal{F}_{2} \in D_{* \mathbf{G}_{\mathbf{O}}}(\mathbf{G r} \times \mathbf{V})$ we have an inductive system

$\ldots \rightarrow \operatorname{RHom}_{D_{* \mathrm{G}_{\mathbf{O}}}(\mathbf{G r} \times \mathbf{V})}\left(\left(\alpha^{n-1}\right)^{*} \mathcal{F}_{1}, \mathcal{F}_{2}\right) \rightarrow \operatorname{RHom}_{D_{* \mathbf{G}_{\mathbf{O}}}}(\mathbf{G r} \times \mathbf{V})\left(\left(\alpha^{n}\right)^{*} \mathcal{F}_{1}, \mathcal{F}_{2}\right) \rightarrow \ldots$

Note that it stabilizes since by definition of $D_{* \mathbf{G}_{\mathbf{O}}}(\mathbf{G r} \times \mathbf{V})$, the restriction of $\left(\alpha^{n}\right)^{*} \mathcal{F}_{1}$ to the support of $\mathcal{F}_{2}$ becomes the pullback of an appropriate sheaf in $D_{\mathbf{G}_{\mathbf{O}}}(\mathbf{G r})$ for $n \gg 0$.

We define the localized category $D_{* \mathbf{G}_{\mathbf{O}}}^{\text {loc }}(\mathbf{G r} \times \mathbf{V})$ as the category with the same objects as $D_{* \mathbf{G}_{\mathbf{O}}}(\mathbf{G r} \times \mathbf{V})$, and with morphisms

$$
\operatorname{RHom}_{D_{* \mathbf{G}_{\mathbf{O}}}^{\mathrm{loc}}(\mathbf{G r} \times \mathbf{V})}\left(\mathcal{F}_{1}, \mathcal{F}_{2}\right):=\lim _{\longrightarrow} \operatorname{RHom}_{D_{* \mathrm{G}_{\mathbf{O}}}(\mathbf{G r} \times \mathbf{V})}\left(\left(\alpha^{n}\right)^{*} \mathcal{F}_{1}, \mathcal{F}_{2}\right)
$$

The tautological functor $D_{* \mathbf{G}_{\mathbf{O}}}(\mathbf{G r} \times \mathbf{V}) \rightarrow D_{* \mathbf{G}_{\mathbf{O}}}^{\text {loc }}(\mathbf{G r} \times \mathbf{V})$ is denoted $u_{0}^{*}$ ("restriction to $v \approx 0 "$ ).

We also have the Verdier dual (to the above $\alpha^{*} \rightarrow$ Id) morphism of endofunctors $\mathrm{Id} \rightarrow \alpha^{!}: D_{\mathbf{G}_{\mathbf{O}}}(\mathbf{G r} \times \mathbf{V}) \rightarrow D_{\mathbf{G}_{\mathbf{O}}}(\mathbf{G r} \times \mathbf{V})$. It gives rise to an inductive system for $\mathcal{F}_{1}, \mathcal{F}_{2} \in D_{! \mathbf{G}_{\mathbf{O}}}(\mathbf{G r} \times \mathbf{V})$

$$
\begin{aligned}
\ldots \rightarrow \operatorname{RHom}_{D_{!_{\mathbf{G}} \mathbf{O}}(\mathbf{G r} \times \mathbf{V})}\left(\mathcal{F}_{1},\left(\alpha^{n-1}\right) ! \mathcal{F}_{2}\right) & \\
& \rightarrow \operatorname{RHom}_{D !_{\mathbf{G}_{\mathbf{O}}}}(\mathbf{G r} \times \mathbf{V}) \\
& \left(\mathcal{F}_{1},\left(\alpha^{n}\right)^{!} \mathcal{F}_{2}\right) \rightarrow \ldots
\end{aligned}
$$

stabilizing for the reasons similar to above. We define the localized category $D_{!_{\mathbf{G}_{\mathbf{O}}}^{\text {loc }}}(\mathbf{G r} \times \mathbf{V})$ as the category with the same objects as $D_{!_{\mathbf{G}_{\mathbf{O}}}}(\mathbf{G r} \times \mathbf{V})$, and with morphisms

$$
\operatorname{RHom}_{D_{! \mathbf{G}_{\mathbf{O}}}^{\text {loc }}(\mathbf{G r} \times \mathbf{V})}\left(\mathcal{F}_{1}, \mathcal{F}_{2}\right):=\lim _{\longrightarrow} \operatorname{RHom}_{D_{!_{\mathbf{G}} \mathbf{O}}(\mathbf{G r} \times \mathbf{V})}\left(\mathcal{F}_{1},\left(\alpha^{n}\right)^{!} \mathcal{F}_{2}\right)
$$

The tautological functor $D_{!_{\mathbf{G}_{\mathbf{O}}}}(\mathbf{G r} \times \mathbf{V}) \rightarrow D_{!_{\mathbf{G}} \mathbf{l o c}}^{\mathrm{loc}}(\mathbf{G r} \times \mathbf{V})$ is denoted $u_{0}^{!}$("corestriction to $v \approx 0 "$ ).

\footnotetext{
${ }^{8}$ terminology of [Sch, 6.2.7]
} 
We also have the projection pr: $\mathbf{G r} \times \mathbf{V}_{0} \rightarrow \mathbf{G r}$, and the corresponding pullbacks pr* $D_{\mathbf{G}_{\mathbf{O}}}(\mathbf{G r}) \rightarrow D_{* \mathbf{G}_{\mathbf{O}}}(\mathbf{G r} \times \mathbf{V}), \mathrm{pr}^{!}: D_{\mathbf{G}_{\mathbf{O}}}(\mathbf{G r}) \rightarrow D_{! \mathbf{G}_{\mathbf{O}}}(\mathbf{G r} \times \mathbf{V})$ (with the essential images supported on $\left.\mathbf{G r} \times \mathbf{V}_{0} \subset \mathbf{G r} \times \mathbf{V}\right)$.

Lemma 3.10.1. The compositions

$$
u_{0}^{*} \circ \mathrm{pr}^{*}: D_{\mathbf{G}_{\mathbf{O}}}(\mathbf{G r}) \rightarrow D_{* \mathbf{G}_{\mathbf{O}}}^{\text {loc }}(\mathbf{G r} \times \mathbf{V}), u_{0}^{!} \circ \mathrm{pr}^{!}: D_{\mathbf{G}_{\mathbf{O}}}(\mathbf{G r}) \rightarrow D_{!_{\mathbf{G}_{\mathbf{O}}}}^{\text {loc }}(\mathbf{G r} \times \mathbf{V})
$$

are equivalences of categories sending $\mathrm{IC}_{0}$ to $\varrho_{\text {right }} E_{0}, \varrho_{\text {right }}^{-1} E_{0}$ respectively.

Proof. Clear.

The localizations of the deequivariantized categories $D_{* \mathbf{G}_{\mathbf{O}}}^{\text {deeq }}(\mathbf{G r} \times \mathbf{V})$, $D_{! \mathbf{G}_{\mathbf{O}}}^{\text {deeq }}(\mathbf{G r} \times \mathbf{V})$ will be denoted $D_{* \mathbf{G}_{\mathbf{O}}}^{\text {loc,deeq }}(\mathbf{G r} \times \mathbf{V})$ and $D_{! \mathbf{G}_{\mathbf{O}}}^{\text {loc,deeq }}(\mathbf{G r} \times \mathbf{V})$ respectively.

Recall the dg-algebras $\mathfrak{A}^{\bullet}, \mathfrak{B}^{\bullet}$ introduced in Section 3.5.

Corollary 3.10.2. There are canonical isomorphisms

(a) $\operatorname{Ext}_{D_{!_{\mathrm{G}} \mathrm{Q}}^{\text {loc, }}(\mathbf{G r} \times \mathbf{V})}^{\bullet}\left(\varrho_{\text {right }}^{-1} E_{0}, \varrho_{\text {right }}^{-1} E_{0}\right) \cong \mathfrak{A}^{\bullet}$,

(b) $\operatorname{Ext}_{D_{* \mathrm{G}_{\mathbf{O}}}^{\bullet}}^{\operatorname{loc,\text {deeq}}(\mathbf{G r} \times \mathbf{V})}\left(\varrho_{\text {right }} E_{0}, \varrho_{\text {right }} E_{0}\right) \cong \mathfrak{B} \bullet$

Note that the dg-algebra $\operatorname{RHom}_{D_{* \mathbf{G}_{\mathbf{O}}}^{\text {deeq }}(\mathbf{G r} \times \mathbf{V})}\left(\varrho_{\text {right }} E_{0}, \varrho_{\text {right }} E_{0}\right) \quad$ (resp. $\left.\operatorname{RHom}_{D_{! \mathbf{G}_{\mathbf{O}}}^{\text {deeq }}(\mathbf{G r} \times \mathbf{V})}\left(\varrho_{\text {right }}^{-1} E_{0}, \varrho_{\text {right }}^{-1} E_{0}\right)\right)$ is also formal, i.e. it is quasiisomorphic to $\operatorname{Ext}_{D_{* \mathbf{G}}^{\text {dee }}(\mathbf{G r} \times \mathbf{V})}^{\bullet}\left(\varrho_{\text {right }} E_{0}, \varrho_{\text {right }} E_{0}\right) \quad\left(\right.$ resp. $\left.\operatorname{Ext}_{D_{! \mathbf{G}_{\mathbf{O}}}^{\text {deeq }}(\mathbf{G r} \times \mathbf{V})}^{\bullet}\left(\varrho_{\text {right }}^{-1} E_{0}, \varrho_{\text {right }}^{-1} E_{0}\right)\right) \quad$ with trivial differential. If we disregard their gradings, they are both isomorphic to $\operatorname{Ext}_{D_{! * \mathbf{G}_{\mathbf{O}}}^{\text {deeq }}(\mathbf{G r} \times \mathbf{V})}^{\bullet}\left(E_{0}, E_{0}\right)$. We will denote the algebra $\operatorname{Ext}_{D_{! * \mathbf{G}_{\mathbf{O}}}^{\text {deeq }}(\mathbf{G r} \times \mathbf{V})}^{\bullet}\left(E_{0}, E_{0}\right)$ with grading forgotten by $\operatorname{Ext}_{D_{! * \mathbf{G}_{\mathbf{O}}}^{\text {deq }}(\mathbf{G r} \times \mathbf{V})}\left(E_{0}, E_{0}\right)$; the same applies to $\mathfrak{A}^{\bullet}, \mathfrak{B}^{\bullet}, \mathfrak{E}^{\bullet}, \mathfrak{G}^{\bullet}$. Thus we have equalities $\operatorname{Ext}_{D_{* \mathrm{G}_{\mathbf{O}}}^{\text {deeq }}(\mathbf{G r} \times \mathbf{V})}\left(\varrho_{\text {right }} E_{0}, \varrho_{\text {right }} E_{0}\right)=$ $\operatorname{Ext}_{D_{! * \mathbf{G}_{\mathbf{O}}}^{\text {deeq }}(\mathbf{G r} \times \mathbf{V})}\left(E_{0}, E_{0}\right)=\operatorname{Ext}_{D_{! \mathbf{G}_{\mathbf{O}}}^{\text {deeq }}(\mathbf{G r} \times \mathbf{V})}\left(\varrho_{\text {right }}^{-1} E_{0}, \varrho_{\text {right }}^{-1} E_{0}\right)$.

Lemma 3.10.3. The natural morphism

$$
\operatorname{Ext}_{D_{! \mathbf{G}_{\mathbf{O}}}^{\text {deeq }}(\mathbf{G r} \times \mathbf{V})}^{\bullet}\left(\varrho_{\text {right }}^{-1} E_{0}, \varrho_{\text {right }}^{-1} E_{0}\right) \rightarrow \operatorname{Ext}_{D_{! \mathbf{G}_{\mathbf{O}}}^{\text {loc,deeq }}(\mathbf{G r} \times \mathbf{V})}^{\bullet}\left(\varrho_{\text {right }}^{-1} E_{0}, \varrho_{\text {right }}^{-1} E_{0}\right)
$$

is injective.

Proof. We have $\alpha \varrho_{\text {right }}^{-1} \mathrm{IC}_{\left(-1^{N}, 1^{N}\right)}[N] \simeq \varrho_{\text {right }}^{-1} E_{0}$, and we need to check that for any $\boldsymbol{\lambda}, \boldsymbol{\mu}$ the natural morphism

$$
\begin{aligned}
\vartheta: \operatorname{RHom}_{D_{! \mathbf{G}_{\mathbf{O}}}}(\mathbf{G r} \times \mathbf{V}) & \left(\varrho_{\text {right }}^{-1} E_{0}, \varrho_{\text {right }}^{-1} \mathrm{IC}_{(\boldsymbol{\lambda}, \boldsymbol{\mu})}\right) \\
& \rightarrow \operatorname{RHom} D_{D_{\mathbf{G}_{\mathbf{O}}}}(\mathbf{G r} \times \mathbf{V}) \\
& \left(\varrho_{\text {right }}^{-1} \mathrm{IC}_{\left(-1^{N}, 1^{N}\right)}[-N], \varrho_{\text {right }}^{-1} \mathrm{IC}_{(\boldsymbol{\lambda}, \boldsymbol{\mu})}\right)
\end{aligned}
$$

is injective. Indeed, since $\alpha^{!}$is a Hecke transformation endofunctor (namely, it is given by convolution $\mathrm{IC}_{\left(1^{N}\right)} *$ ? $* \mathrm{IC}_{(-1)^{N}}$ with invertible objects of the Satake 
category), the morphism $\vartheta$ is an endomorphism of the identity endofunctor of the deequivariantized category $D_{! \mathbf{G}_{\mathbf{O}}}^{\text {deeq }}(\mathbf{G r} \times \mathbf{V})$. In other words, $\vartheta$ is an element of the center of this category. But the natural morphism from the center to its localization is injective iff the multiplication by $\vartheta$ is injective.

We change the setting to the base field $\mathbb{F}_{q}$ as in the proof of Lemma 3.9.1. Then all the IC sheaves in question carry a natural Weil structure, and it was proved in loc. cit. that both the LHS and the RHS of (3.10.1) are pure; it is immediate to see that they are pure of the same weight $w$. The cone of $\vartheta$ is $H_{\mathbf{G}_{\mathbf{O}}}^{\bullet}\left(\Omega_{\left(0^{N}, 0^{N}\right)}, \imath_{0}^{!} \varrho_{\text {right }}^{-1} \mathrm{IC}_{(\boldsymbol{\lambda}, \boldsymbol{\mu})}\right)$ where $\iota_{0}$ stands for the locally closed embedding of

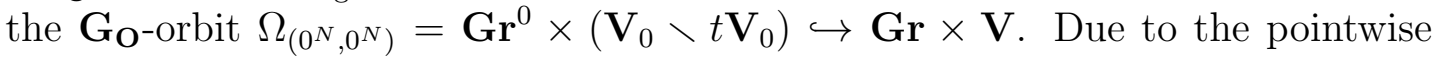
purity of $\mathrm{IC}_{(\boldsymbol{\lambda}, \boldsymbol{\mu})}$ [FGT, Section 3], $\imath_{0}^{!} \mathrm{IC}_{(\boldsymbol{\lambda}, \boldsymbol{\mu})}$ is a pure local system on $\Omega_{\left(0^{N}, 0^{N}\right)}$; hence $H_{\mathbf{G}_{\mathbf{O}}}^{\bullet}\left(\Omega_{\left(0^{N}, 0^{N}\right)}, \imath_{0}^{!} \mathrm{IC}_{(\boldsymbol{\lambda}, \boldsymbol{\mu})}\right)$ is also pure of weight $w$. It follows that the kernel of $\vartheta$ vanishes, and $\vartheta$ is injective.

Corollary 3.10.4. The algebra $\mathfrak{E}=\operatorname{Ext}_{D_{!_{*} \mathbf{G}_{\mathbf{O}}}^{\text {deeq }}(\mathbf{G r} \times \mathbf{V})}\left(E_{0}, E_{0}\right)$ is a commutative integrally closed domain.

3.11. Calculation of the Ext algebra. Recall that the first fundamental coweight of $\mathrm{GL}_{N}$ is $\omega_{1}=(1,0, \ldots, 0)$, and $\omega_{1}^{*}=(0, \ldots, 0,-1)$. We have $\mathrm{RHom}_{D_{! * \mathrm{G}_{\mathbf{O}}}}(\mathbf{G r} \times \mathbf{V})\left(\mathrm{IC}_{\omega_{1}} * E_{0}, E_{0} * \mathrm{IC}_{\omega_{1}}\right)=\mathrm{RHom}_{D_{! * \mathrm{G}_{\mathbf{O}}}}(\mathbf{G r} \times \mathbf{V})\left(E_{0}, \mathrm{IC}_{\omega_{1}^{*}} * E_{0} * \mathrm{IC}_{\omega_{1}}\right)$,

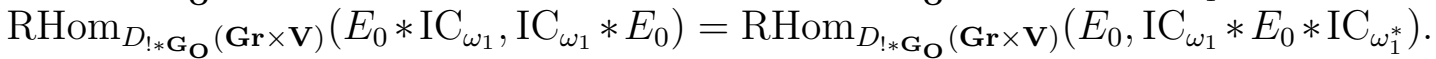
Now $\mathrm{IC}_{\omega_{1}} * E_{0}$ is the constant IC-sheaf of the stratum closure formed by all the pairs $(L, v)$ such that the lattice $L$ contains $\mathbf{V}_{0}$ as a hyperplane, and $v \in L$. Furthermore, $E_{0} * \mathrm{IC}_{\omega_{1}}$ is the constant IC-sheaf of the stratum closure formed by all the pairs $(L, v)$ such that the lattice $L$ contains $\mathbf{V}_{0}$ as a hyperplane, and $v \in \mathbf{V}_{0}$. In particular, the latter stratum closure is a smooth divisor in the former stratum closure, so we have canonical elements $h \in \operatorname{Ext}_{D_{! * \mathbf{G}_{\mathbf{O}}}(\mathbf{G r} \times \mathbf{V})}^{1}\left(\mathrm{IC}_{\omega_{1}} * E_{0}, E_{0} * \mathrm{IC}_{\omega_{1}}\right)$

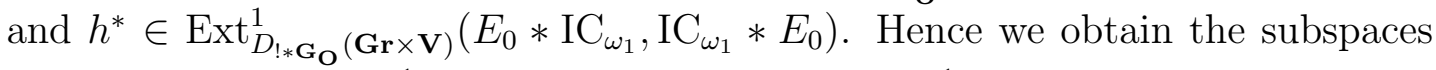
$h \otimes V_{\omega_{1}^{*}} \otimes V_{\omega_{1}} \subset \mathfrak{E}^{1}$ and $h^{*} \otimes V_{\omega_{1}} \otimes V_{\omega_{1}^{*}} \subset \mathfrak{E}^{1}$ (see Section 3.9 for the definition of $\mathfrak{E}^{\bullet}$ and (3.9.1)). We identify the former subspace with $\operatorname{Hom}\left(V_{1}, V_{2}\right)$ and the latter one with $\operatorname{Hom}\left(V_{2}, V_{1}\right)$. Thus we obtain a homomorphism $\phi \bullet: \operatorname{Sym}\left(\Pi \operatorname{Hom}\left(V_{1}, V_{2}\right)[-1] \oplus \Pi \operatorname{Hom}\left(V_{2}, V_{1}\right)[-1]\right)=\operatorname{Sym}\left(\mathfrak{g}_{1}[-1]\right)=\mathfrak{G}_{1,1}^{\bullet} \rightarrow \mathfrak{E}^{\bullet}$ (due to commutativity of $\mathfrak{E}$ ).

Lemma 3.11.1. $\phi^{\bullet}$ is an isomorphism.

Proof. We can and will disregard the grading. The morphism $\phi$ induces the morphism $\phi^{*}:$ Spec $\mathfrak{E} \rightarrow \mathfrak{g}_{1}^{*}$ that is an isomorphism over the open subset $\operatorname{Isom}\left(V_{2}, V_{1}\right) \times \operatorname{Hom}\left(V_{1}, V_{2}\right) \subset \mathfrak{g}_{1}^{*}$ due to Corollary 3.10.2(a). Similarly, $\phi^{*}$ is an isomorphism over the open subset $\operatorname{Hom}\left(V_{2}, V_{1}\right) \times \operatorname{Isom}\left(V_{1}, V_{2}\right)$ due to Corollary 3.10.2(b). 
Since the complement to the union of these two open subsets has codimension 2 in $\mathfrak{g}_{1}^{*}$, we can apply Lemma 3.11.2 below. Note that the irreducibility of Spec $\mathfrak{E}$ is guaranteed by Corollary 3.10.4. It remains only to check that the ratio of the above isomorphisms is the identity birational isomorphism between the varieties $\operatorname{Isom}\left(V_{2}, V_{1}\right) \times \operatorname{Hom}\left(V_{1}, V_{2}\right)$ and $\operatorname{Hom}\left(V_{2}, V_{1}\right) \times \operatorname{Isom}\left(V_{1}, V_{2}\right)$.

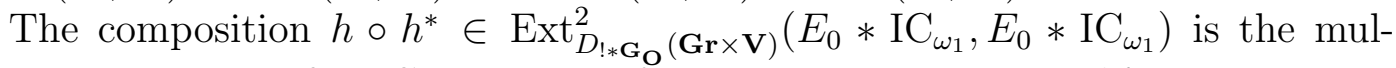
tiplication by the first Chern class of the normal line bundle $\mathcal{N}$ to the divisor $\operatorname{supp}\left(E_{0} * \mathrm{IC}_{\omega_{1}}\right)$ in $\operatorname{supp}\left(\mathrm{IC}_{\omega_{1}} * E_{0}\right)$. Recall that $\mathbf{G r}^{\omega_{1}} \simeq \mathbb{P}^{N-1}$. The line bundle $\mathcal{N}$ is pulled back from the line bundle $\mathcal{O}(1)$ on $\mathbb{P}^{N-1} \simeq \mathbf{G r}^{\omega_{1}}$. Recall also that the restriction of the determinant line bundle $\mathcal{L}$ from $\mathbf{G r}$ to $\mathbf{G r}^{\omega_{1}}$ is also isomorphic to $\mathcal{O}(1)$. We conclude that $h \circ h^{*}=c_{1}(\mathcal{L})$.

On the other hand, in the equivariant Satake category

$$
D_{\mathbf{G}_{\mathbf{O}}}(\mathbf{G r}) \cong D_{\text {perf }}^{\mathrm{GL}_{N}}\left(\operatorname{Sym}\left(\mathfrak{g l}_{N}[-2]\right)\right) \cong D_{\text {perf }}^{G_{\overline{0}}}\left(\mathfrak{A}^{\bullet}\right),
$$

the first Chern class $c_{1}(\mathcal{L}) \in \operatorname{Ext}_{D_{\mathrm{G}_{\mathbf{O}}}(\mathbf{G r})}^{2}\left(\mathrm{IC}_{0} * \mathrm{IC}_{\omega_{1}}, \mathrm{IC}_{0} * \mathrm{IC}_{\omega_{1}}\right) \subset \mathfrak{A}^{2}$ corresponds to the identity element (shifted by 2 ) Id $\in \operatorname{Hom}\left(V_{2}, V_{1}\right)^{*} \otimes \operatorname{Hom}\left(V_{2}, V_{1}\right)$.

The lemma is proved.

Lemma 3.11.2. Let $\pi: X \rightarrow \mathbb{A}^{n}$ be a morphism from an irreducible affine algebraic variety to an affine space. Let $f, g \in \mathbb{C}\left[\mathbb{A}^{n}\right]$ be such that the codimension of the closed subvariety $\mathbb{A}^{n} \backslash\left(U_{f} \cup U_{g}\right)$ in $\mathbb{A}^{n}$ is at least 2 , where $U_{f}=\left\{u \in \mathbb{A}^{n} \mid f(u) \neq 0\right\}$. Assume moreover that each of the morphisms $\pi^{-1}\left(U_{f}\right) \rightarrow U_{f}$ and $\pi^{-1}\left(U_{g}\right) \rightarrow U_{g}$, induced by $\pi$, is an isomorphism. Then $\pi$ is an isomorphism.

Proof. Let $X_{f}=\pi^{-1}\left(U_{f}\right)$, resp. $X_{g}=\pi^{-1}\left(U_{g}\right)$, and write $j: U_{f} \cup U_{g} \hookrightarrow \mathbb{A}^{n}$, resp. $j_{X}: X_{f} \cup X_{g} \hookrightarrow X$, for the open imbedding. We have the following commutative diagram

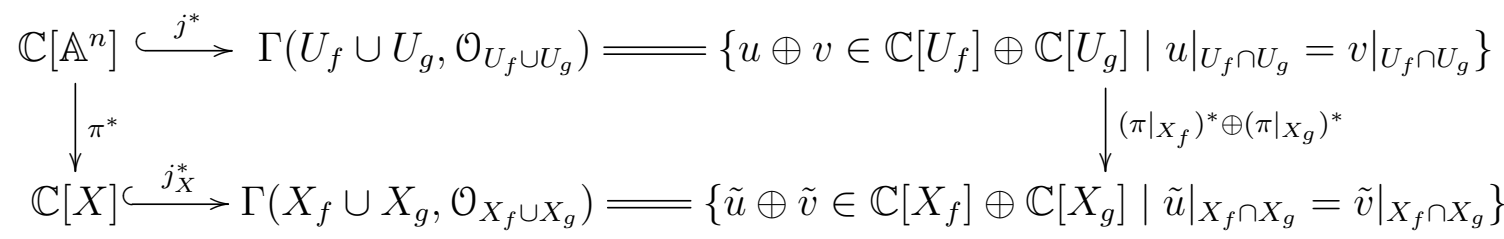

The map $j^{*}$ in this diagram is an isomorphism by the codimension $\geq 2$ assumption. The map $j_{X}^{*}$ is injective since $X$ is irreducible. The assumptions imply also that the vertical map $\left(\left.\pi\right|_{X_{f}}\right)^{*} \oplus\left(\left.\pi\right|_{X_{g}}\right)^{*}$ on the right is an isomorphism. It follows that the vertical map $\pi^{*}$ on the left must be an isomorphism, as required.

3.12. Restriction to $\mathbf{G r} \times \mathbf{V}_{0}$. The existence of the desired equivalence $\Phi^{1,1}$ of Theorem 3.6.1 follows from Lemma 3.9.2 and Lemma 3.11.1. The equivalences 
$\Phi^{0,2}$ and $\Phi^{2,0}$ are obtained by conjugating with $\varrho_{\text {right }}^{ \pm 1}$. It remains to check their compatibility with monoidal structures.

We denote by $\bar{\jmath}_{0}\left(\right.$ resp. $\left.\bar{\jmath}_{-1}\right)$ the closed embedding $\mathbf{G r} \times \mathbf{V}_{0} \hookrightarrow \mathbf{G r} \times \mathbf{V}$ (resp. $\left.\mathbf{G r} \times t \mathbf{V}_{0} \hookrightarrow \mathbf{G r} \times \mathbf{V}\right)$. We also denote by $\jmath_{0}$ the locally closed embedding $\mathbf{G r} \times\left(\mathbf{V}_{0} \backslash t \mathbf{V}_{0}\right) \hookrightarrow \mathbf{G r} \times \mathbf{V}$. Our goal in this section is a description in terms of $\Phi^{1,1}$ of the endofunctors $\bar{\jmath}_{0 *} \bar{\jmath}_{0}^{!}, \jmath_{0 *} \jmath_{0}^{!}: D_{! * \mathbf{G}_{\mathbf{O}}}(\mathbf{G r} \times \mathbf{V}) \rightarrow D_{! * \mathbf{G}_{\mathbf{O}}}(\mathbf{G r} \times \mathbf{V})$.

Recall the setup and notation of Section 3.4. We have the natural morphisms

$$
\begin{gathered}
p: Q^{A} \rightarrow \operatorname{Hom}\left(V_{1}, V_{2}\right) \times \operatorname{Hom}\left(V_{2}, V_{1}\right)=\Pi \mathfrak{g}_{1}^{*}, \\
q: Q^{A} \rightarrow \operatorname{Hom}\left(V_{1}^{\prime}, V_{2}\right) \times \operatorname{Hom}\left(V_{2}, V_{1}^{\prime}\right)=\Pi \mathfrak{g}_{1}^{*} ; \\
p\left(A, A^{\prime}, A^{\prime \prime}, B, B^{\prime}, B^{\prime \prime}\right)=(A, B), q\left(A, A^{\prime}, A^{\prime \prime}, B, B^{\prime}, B^{\prime \prime}\right)=\left(A^{\prime}, B^{\prime}\right) .
\end{gathered}
$$

We also have the natural morphisms

$$
p, q: G_{\mathcal{Q}} \rightarrow G_{\overline{0}}, p\left(g_{1}, g_{1}^{\prime}, g_{2}\right)=\left(g_{1}, g_{2}\right), q\left(g_{1}, g_{1}^{\prime}, g_{2}\right)=\left(g_{1}^{\prime}, g_{2}\right) \text {. }
$$

Clearly, $p, q: \mathcal{Q}^{A} \rightarrow \Pi \mathfrak{g}_{1}^{*}$ are equivariant with respect to $p, q: G_{\mathcal{Q}} \rightarrow G_{\overline{0}}$. Hence we have the convolution functor

$p_{*} q^{*}: \operatorname{Coh}^{G_{\overline{0}}}\left(\Pi \mathfrak{g}_{1}^{*}\right)=\operatorname{Coh}\left(G_{\overline{0}} \backslash \Pi \mathfrak{g}_{1}^{*}\right) \stackrel{q^{*}}{\rightarrow} \operatorname{Coh}\left(G_{\mathcal{Q}} \backslash Q^{A}\right) \stackrel{p_{*}}{\rightarrow} \operatorname{Coh}\left(G_{\overline{0}} \backslash \Pi \mathfrak{g}_{1}^{*}\right)=\operatorname{Coh}^{G_{\overline{0}}}\left(\Pi \mathfrak{g}_{1}^{*}\right)$

(in particular, $p_{*}$ involves taking $\mathrm{GL}\left(V_{1}^{\prime}\right)$-invariants). We will actually need the same named functor $p_{*} q^{*}: D_{\text {perf }}^{G_{\overline{0}}}\left(\mathfrak{G}_{1,1}^{\bullet}\right) \rightarrow D_{\text {perf }}^{G_{\overline{0}}}\left(\mathfrak{G}_{1,1}^{\bullet}\right)$ defined similarly using the dg-algebra with trivial differential $\mathfrak{G}_{1,1}^{\bullet} \otimes \mathbb{C}\left[\operatorname{Hom}\left(V_{1}, V_{1}^{\prime}\right)\right]$ (the grading on $\mathbb{C}\left[\operatorname{Hom}\left(V_{1}, V_{1}^{\prime}\right)\right]$ is trivial, and if we disregard the grading, then $\left.\mathfrak{G}_{1,1}^{\bullet} \otimes \mathbb{C}\left[\operatorname{Hom}\left(V_{1}, V_{1}^{\prime}\right)\right] \simeq \mathbb{C}\left[Q^{A}\right]\right)$.

We also have a $G_{Q}$-invariant subvariety $\mathcal{Q}_{0}^{A} \subset Q^{A}$ given by the equation that $A^{\prime \prime}$ is noninvertible. The restriction of $p, q$ to $Q_{0}^{A}$ will be denoted by $p_{0}, q_{0}$. As above, we obtain the functor $p_{0 *} q_{0}^{*}: D_{\text {perf }}^{G_{\overline{0}}}\left(\mathfrak{G}_{1,1}^{\bullet}\right) \rightarrow D_{\text {perf }}^{G_{\overline{0}}}\left(\mathfrak{G}_{1,1}^{\bullet}\right)$.

Proposition 3.12.1. (a) There is an isomorphism of functors

$$
\bar{\jmath}_{0 *} \bar{\jmath}_{0}^{!} \circ \Phi^{1,1} \simeq \Phi^{1,1} \circ p_{*} q^{*}: D_{\text {perf }}^{G_{\overline{0}}}\left(\mathfrak{G}_{1,1}^{\bullet}\right) \rightarrow D_{! * \mathbf{G}_{\mathbf{O}}}(\mathbf{G r} \times \mathbf{V}) .
$$

(b) The isomorphism in (a) can be extended to the following commutative diagram of morphisms:

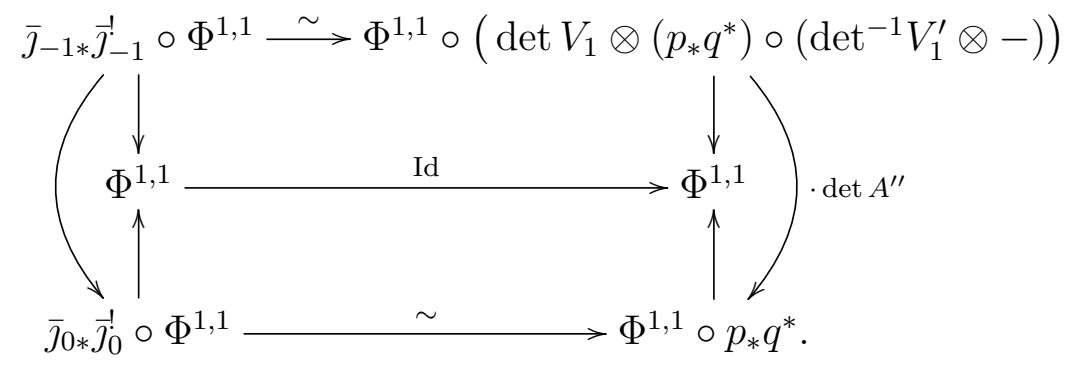

(c) There is an isomorphism of functors

$$
\jmath_{0 *} \jmath_{0}^{!} \circ \Phi^{1,1} \simeq \Phi^{1,1} \circ p_{0 *} q_{0}^{*}: D_{\text {perf }}^{G_{\overline{0}}}\left(\mathfrak{G}_{1,1}^{\bullet}\right) \rightarrow D_{! * \mathbf{G}_{\mathbf{O}}}(\mathbf{G r} \times \mathbf{V})
$$


Proof. (a) The support of $\mathrm{IC}_{(\boldsymbol{\nu}, \boldsymbol{\mu})}$ lies in $\mathbf{G r} \times \mathbf{V}_{0}$ iff $\boldsymbol{\nu}$ is a negative partition: $\left(0 \geq \nu_{1} \geq \ldots \geq \nu_{N}\right)$ (see [FGT, Proof of Proposition 8]). So $D_{!_{*} \mathbf{G}_{\mathbf{o}}}\left(\mathbf{G r} \times \mathbf{V}_{0}\right)$ is generated by the collection of objects $\mathrm{IC}_{(\boldsymbol{\nu}, \boldsymbol{\mu})}$ where $\boldsymbol{\nu}$ is a negative partition. Thus we see that $\bar{\jmath}_{0 *}$ is a fully faithful functor whose image is generated by $\left\{\mathrm{IC}_{(\boldsymbol{\nu}, \boldsymbol{\mu})} \mid \boldsymbol{\nu} \leq 0\right\}$ and $\bar{\jmath}_{0}^{!}$is the right adjoint of $\bar{\jmath}_{0 *}$. Recall that $V_{\boldsymbol{\nu}}^{*}$ denotes an irreducible representation of $\mathrm{GL}(V)$ obtained by applying the corresponding Schur functor to $V^{*}$. The result of application of the same Schur functor to $V_{1}^{*}$ $\left(\right.$ resp. $\left.V_{1}^{\prime *}, V_{2}^{*}\right)$ will be denoted $V_{1, \boldsymbol{\nu}}^{*}\left(\operatorname{resp} . V_{1, \boldsymbol{\nu}}^{\prime *}, V_{2, \boldsymbol{\nu}}^{*}\right)$. Since

$$
\mathrm{IC}_{(\boldsymbol{\nu}, \boldsymbol{\mu})}=\Phi^{1,1}\left(V_{1, \boldsymbol{\nu}}^{*} \otimes \mathfrak{G}_{1,1}^{\bullet} \otimes V_{2, \boldsymbol{\mu}}^{*}\right)=\Phi^{1,1}\left(V_{1, \boldsymbol{\nu}^{*}} \otimes \mathfrak{G}_{1,1}^{\bullet} \otimes V_{2, \boldsymbol{\mu}^{*}}\right)
$$

(where $\boldsymbol{\nu}^{*}=\left(-\nu_{N},-\nu_{N-1}, \ldots,-\nu_{2},-\nu_{1}\right)$ for $\boldsymbol{\nu}=\left(\nu_{1}, \ldots, \nu_{N}\right)$ ), we have to show that $p_{*} q^{*}$ lands in the subcategory $D_{\text {perf }}^{G_{\overline{0}}, \geq}\left(\mathfrak{G}_{1,1}^{\bullet}\right) \subset D_{\text {perf }}^{G_{\overline{0}}}\left(\mathfrak{G}_{1,1}^{\bullet}\right)$ generated by objects $\left\{V_{1, \boldsymbol{\lambda}} \otimes \mathfrak{G}_{1,1}^{\bullet} \otimes V_{2, \boldsymbol{\mu}} \mid \boldsymbol{\lambda} \geq 0\right\}$ and to construct the adjunction isomorphism

$$
\begin{aligned}
& \operatorname{Hom}_{D_{\text {perf }}^{G_{\overline{0}}\left(\mathfrak{G}_{1,1}\right)}}\left(V_{1, \boldsymbol{\lambda}} \otimes \mathfrak{G}_{1,1}^{\bullet} \otimes V_{2, \boldsymbol{\mu}}, V_{1, \boldsymbol{\lambda}^{\prime}} \otimes \mathfrak{G}_{1,1}^{\bullet} \otimes V_{2, \boldsymbol{\mu}^{\prime}}\right) \\
& \stackrel{\sim}{\longrightarrow} \operatorname{Hom}_{D_{\text {perf }}^{G_{\overline{0}}\left(\mathfrak{G}_{i, 1}^{\bullet}\right)}}\left(V_{1, \boldsymbol{\lambda}} \otimes \mathfrak{G}_{1,1}^{\bullet} \otimes V_{2, \boldsymbol{\mu}}, p_{*} q^{*}\left(V_{1, \boldsymbol{\lambda}^{\prime}}^{\prime} \otimes \mathfrak{G}_{1,1}^{\bullet} \otimes V_{2, \boldsymbol{\mu}^{\prime}}\right)\right)
\end{aligned}
$$

for a partition $\boldsymbol{\lambda}$. Equivalently, we have to construct an isomorphism

$$
\begin{gathered}
\left(\left(V_{1, \boldsymbol{\lambda}}^{*} \otimes V_{1, \boldsymbol{\lambda}^{\prime}}^{\prime}\right) \otimes \mathbb{C}\left[\operatorname{Hom}\left(V_{1}^{\prime}, V_{2}\right) \times \operatorname{Hom}\left(V_{2}, V_{1}^{\prime}\right)\right] \otimes\left(V_{2, \boldsymbol{\mu}}^{*} \otimes V_{2, \boldsymbol{\mu}^{\prime}}\right)\right)^{\mathrm{GL}\left(V_{1}^{\prime}\right) \times \mathrm{GL}\left(V_{2}\right)} \\
\stackrel{\sim}{\longrightarrow}\left(V_{1, \boldsymbol{\lambda}}^{*} \otimes p_{*} q^{*}\left(V_{1, \boldsymbol{\lambda}^{\prime}}^{\prime} \otimes \mathbb{C}\left[\operatorname{Hom}\left(V_{1}^{\prime}, V_{2}\right) \times \operatorname{Hom}\left(V_{2}, V_{1}^{\prime}\right)\right] \otimes V_{2, \boldsymbol{\mu}^{\prime}}\right) \otimes V_{2, \boldsymbol{\mu}}^{*}\right)^{\mathrm{GL}\left(V_{1}\right) \times \mathrm{GL}\left(V_{2}\right)} \\
:=\left(V_{1, \boldsymbol{\lambda}}^{*} \otimes V_{1, \boldsymbol{\lambda}^{\prime}}^{\prime} \otimes \mathbb{C}\left[Q^{A}\right] \otimes\left(V_{2, \boldsymbol{\mu}}^{*} \otimes V_{2, \boldsymbol{\mu}^{\prime}}\right)\right)^{G_{Q}} .
\end{gathered}
$$

Recall that $Q^{A}=\operatorname{Hom}\left(V_{1}, V_{1}^{\prime}\right) \times \operatorname{Hom}\left(V_{1}^{\prime}, V_{2}\right) \times \operatorname{Hom}\left(V_{2}, V_{1}\right)$. We apply Lemma 3.13.1(c) below to $U_{1}=V_{2}, U_{2}=V_{1}, U_{3}=V_{1}^{\prime}, \boldsymbol{\nu}=\boldsymbol{\lambda}$ (notation of 3.13) to obtain an isomorphism

$$
V_{1, \boldsymbol{\lambda}}^{\prime *} \otimes \mathbb{C}\left[\operatorname{Hom}\left(V_{2}, V_{1}^{\prime}\right)\right] \stackrel{\sim}{\sim}\left(\mathbb{C}\left[\operatorname{Hom}\left(V_{2}, V_{1}\right) \otimes V_{1, \boldsymbol{\lambda}}^{*} \otimes \mathbb{C}\left[\operatorname{Hom}\left(V_{1}, V_{1}^{\prime}\right)\right]\right)^{\mathrm{GL}\left(V_{1}\right)}\right.
$$

whose inverse induces the desired adjunction isomorphism.

We still have to check that the essential image of $p_{*} q^{*}$ lies in the subcategory $D_{\text {perf }}^{G_{\overline{0}}, \geq}\left(\mathfrak{G}_{1,1}^{\bullet}\right) \subset D_{\text {perf }}^{G_{\overline{0}}}\left(\mathfrak{G}_{1,1}^{\bullet}\right)$ generated by $\left\{V_{1, \boldsymbol{\lambda}} \otimes \mathfrak{G}_{1,1}^{\bullet} \otimes V_{2, \boldsymbol{\mu}} \mid \boldsymbol{\lambda} \geq 0\right\}$. We consider the homomorphism $\mathfrak{G}_{1,1}^{\bullet} \rightarrow \mathbb{C}$ killing all the generators, and for $\mathcal{M} \in D_{\text {perf }}^{G_{\overline{0}}}\left(\mathfrak{G}_{1,1}{ }_{1,1}\right)$

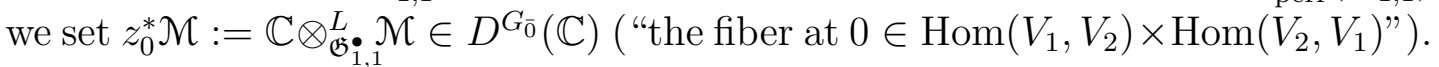
Note that $z_{0}^{*} p_{*} q^{*}$ lands in the category generated by $\left\{V_{1, \boldsymbol{\lambda}} \otimes V_{2, \boldsymbol{\mu}} \mid \boldsymbol{\lambda} \geq 0\right\}$, i.e. the category of modules with polynomial action of $\operatorname{GL}\left(V_{1}\right)$. Indeed, recall that $G_{\mathcal{Q}}$ acts on $\mathcal{Q}^{A}$ via

$$
\left(g_{1}, g_{1}^{\prime}, g_{2}\right)\left(A^{\prime}, A^{\prime \prime}, B\right)=\left(g_{2} A^{\prime} g_{1}^{\prime-1}, g_{1}^{\prime} A^{\prime \prime} g_{1}^{-1}, g_{1} B g_{2}^{-1}\right) .
$$

Since we impose the conditions $B=0=A:=A^{\prime} A^{\prime \prime}$, the action of $\mathrm{GL}\left(V_{1}\right)$ on $z_{0}^{*} p_{*} q^{*} \mathcal{N}$ (for a free $\operatorname{dg}-\mathfrak{G}_{1,1}^{\bullet}$-module $\mathcal{N}$ ) comes from its action on functions of $A^{\prime \prime}$, and the latter action is polynomial. 
Finally, we claim that if the action of $\mathrm{GL}\left(V_{1}\right)$ on $z_{0}^{*} \mathcal{M}$ is polynomial, then $\mathcal{M}$ lies in the subcategory generated by $\left\{V_{1, \boldsymbol{\lambda}} \otimes \mathfrak{G}_{1,1}^{\bullet} \otimes V_{2, \boldsymbol{\mu}} \mid \boldsymbol{\lambda} \geq 0\right\}$. To this end we apply the Koszul equivalence $\varkappa: D_{\text {fd }}^{G_{\overline{0}}}(\Lambda) \stackrel{\sim}{\longrightarrow} D_{\text {perf }}^{G_{\overline{0}}}\left(\mathfrak{G}_{1,1}^{\bullet}\right)$ of Section 3.8. It is easy to see that if the action of $\operatorname{GL}\left(V_{1}\right)$ on the total cohomology of $\mathcal{K} \in D_{\mathrm{fd}}^{G_{\overline{0}}}(\Lambda)$ is polynomial, then $\mathcal{K}$ lies in the subcategory Pol $\subset D_{\mathrm{fd}}^{G_{\overline{0}}}(\Lambda)$ generated by the $G_{\overline{0}} \ltimes \Lambda$-modules with polynomial action of $\operatorname{GL}\left(V_{1}\right)$ and trivial action of $\Lambda$. Now $\varkappa(\mathrm{Pol})$ is the subcategory $D_{\text {perf }}^{G_{\overline{0}} \geq}\left(\mathfrak{G}_{1,1}^{\bullet}\right) \subset D_{\text {perf }}^{G_{\overline{0}}}\left(\mathfrak{G}_{1,1}^{\bullet}\right)$. And if the action of $\operatorname{GL}\left(V_{1}\right)$ on $z_{0}^{*} \mathcal{M}$ is polynomial, then $\mathcal{M} \simeq \varkappa(\mathcal{K})$, where the action of $\operatorname{GL}\left(V_{1}\right)$ on the total cohomology of $\mathcal{K}$ is polynomial. This completes the proof of (a).

(b) As $\alpha^{*} \bar{\jmath}_{-1 *} \bar{\jmath}_{-1}^{!} \simeq \bar{\jmath}_{0 *} \bar{J}_{0}^{!} \alpha^{*}$ (notation of Section 3.10), we deduce an isomorphism of functors

$\bar{\jmath}_{-1 *} \bar{\jmath}_{-1}^{!} \circ \Phi^{1,1} \simeq \Phi^{1,1} \circ\left(\operatorname{det} V_{1} \otimes\left(p_{*} q^{*}\right) \circ\left(\operatorname{det}^{-1} V_{1}^{\prime} \otimes-\right)\right): D_{\operatorname{perf}}^{G_{\overline{0}}}\left(\mathfrak{G}_{1,1}^{\bullet}\right) \rightarrow D_{! * \mathbf{G}_{\mathbf{O}}}(\mathbf{G r} \times \mathbf{V})$.

Thus the upper and lower rectangles of the diagram in (b) are commutative by construction. We have to prove the commutativity of the big curved quadrangle. The endofunctors $\bar{\jmath}_{0 *} \bar{J}_{0}^{!}, \bar{\jmath}_{-1 *} \bar{J}_{-1}^{!}$of $D_{! * \mathbf{G}_{\mathbf{O}}}(\mathbf{G r} \times \mathbf{V})$ are equipped with the structure of idempotent comonads. The desired commutativity follows from the fact that given two idempotent comonads $T_{0}, T_{1}: \mathcal{C} \rightarrow \mathcal{C}$, there is at most one morphism of functors $\chi: T_{1} \rightarrow T_{0}$ such that $\varepsilon_{1}=\varepsilon_{0} \circ \chi$ for the counits $\varepsilon_{i}: T_{i} \rightarrow \operatorname{Id} \mathrm{d}_{\mathcal{C}}$. Indeed, $\chi=T_{0}\left(\varepsilon_{1}\right) \circ \chi_{1}$, where $\chi_{1}$ is defined as the composition

$$
T_{1} \cong T_{1} \circ T_{1} \stackrel{\chi \circ T_{1}}{\longrightarrow} T_{0} \circ T_{1}
$$

We claim that $\chi_{1}$ is an isomorphism uniquely defined as the inverse to the morphism $\varepsilon_{0} \circ T_{1}: T_{0} \circ T_{1} \stackrel{\sim}{\longrightarrow} T_{1}$. In effect, the composition

$$
T_{1} \cong T_{1} \circ T_{1} \stackrel{\chi \circ T_{1}}{\longrightarrow} T_{0} \circ T_{1} \stackrel{\varepsilon_{0} \circ T_{1}}{\longrightarrow} T_{1}
$$

equals the composition

$$
T_{1} \cong T_{1} \circ T_{1} \stackrel{\varepsilon_{1} \circ T_{1}}{\longrightarrow} \operatorname{Id}_{\mathcal{C}} \circ T_{1}=T_{1}
$$

that in turn equals $\operatorname{Id}_{T_{1}}$. Conversely, the composition

$$
T_{0} \circ T_{1} \stackrel{\varepsilon_{0} \circ T_{1}}{\longrightarrow} T_{1} \cong T_{1} \circ T_{1} \stackrel{\chi \circ T_{1}}{\longrightarrow} T_{0} \circ T_{1}
$$

equals the composition

$$
T_{0} \circ T_{1} \cong T_{0} \circ T_{1} \circ T_{1} \stackrel{T_{0} \circ \chi \circ T_{1}}{\longrightarrow} T_{0} \circ T_{0} \circ T_{1} \cong T_{0} \circ T_{1}
$$

that in turn equals $\operatorname{Id}_{T_{0} \circ T_{1}}$.

This completes the proof of (b), but we would like to give one more independent argument that will prove useful later on.

Recall that we have to prove the commutativity of the big curved quadrangle. To this end we change the setting to the base field $\mathbb{F}_{q}$ as in the proof of 
Lemma 3.9.1. That is we replace $D_{! * \mathbf{G}_{\mathbf{O}}}(\mathbf{G r} \times \mathbf{V})$ by the equivalent equivariant derived category of sheaves on $(\mathbf{G r} \times \mathbf{V})_{\overline{\mathbb{F}}_{q}}$ as in [BF, Proposition 5] (in particular, choosing an isomorphism $\mathbb{C} \simeq \overline{\mathbb{Q}}_{\ell}$ ). However, we preserve the notation $D_{! * \mathbf{G}_{\mathbf{O}}}(\mathbf{G r} \times \mathbf{V})$ for this category in order not to overload our notation (anyway, it will only be used during the current proof). All the irreducible perverse sheaves $\mathrm{IC}_{(\boldsymbol{\lambda}, \boldsymbol{\mu})}$ carry a natural Tate Weil structure by [FGT, Proposition 11]. They (along with their Tate twists) generate a subcategory ${ }^{9} \widehat{D}_{! * \mathbf{G}_{\mathbf{O}}}(\mathbf{G r} \times \mathbf{V})$ of the mixed version $D_{! * \mathbf{G}_{\mathbf{O}}}^{\text {mix }}(\mathbf{G r} \times \mathbf{V})$ of $D_{! * \mathbf{G}_{\mathbf{O}}}(\mathbf{G r} \times \mathbf{V})$. We will use a particular dgmodel of $\widehat{D}_{! * \mathbf{G}_{\mathbf{O}}}(\mathbf{G r} \times \mathbf{V})$ viewed as a category enriched over complexes equipped with an action of the Frobenius automorphism Fr. Note that the absolute values of the eigenvalues of Fr lie in $\sqrt{q}^{\mathbb{Z}}$, and hence our complexes carry an additional grading according to the absolute values of the eigenvalues of Fr. If we forget the mixed structure and remember only this additional grading, we obtain a category $\widetilde{\widetilde{D}}_{: * \mathbf{G}_{\mathbf{O}}}(\mathbf{G r} \times \mathbf{V})$ enriched over complexes equipped with an additional grading. Its localization with respect to quasiisomorphisms will be denoted $\widetilde{D}_{! * \mathbf{G}_{\mathbf{O}}}(\mathbf{G r} \times \mathbf{V})$.

On the other hand, we consider the category $\underline{D}_{\text {perf }}^{G_{\overline{0}} \times \mathbb{C}^{\times}}\left(\mathfrak{G}_{1,1}^{\bullet}\right)$ of perfect $G_{\overline{0}} \times \mathbb{C}^{\times}$-equivariant dg- $\mathfrak{G}_{1,1}^{\bullet}$-modules and its localization (with respect to quasi-isomorphisms) $D_{\text {perf }}^{G_{\overline{0}} \times \mathbb{C}^{\times}}\left(\mathfrak{G}_{1,1}^{\bullet}\right)$. Here all the generators of $\mathfrak{G}_{1,1}^{\bullet}$ have weight 1 with respect to the action of $\mathbb{C}^{\times}$. Then the standard modification of our construction of the equivalence $\Phi^{1,1}: D_{\text {perf }}^{G_{\overline{0}}}\left(\mathfrak{G}_{1,1}^{\bullet}\right) \stackrel{\sim}{\longrightarrow} D_{! * \mathbf{G}_{\mathbf{O}}}(\mathbf{G r} \times \mathbf{V})$ produces a functor $\underline{\widetilde{\Phi}}^{1,1}: \underline{D}_{\mathrm{perf}}^{G_{\overline{0}} \times \mathbb{C}^{\times}}\left(\mathfrak{G}_{1,1}^{\bullet}\right) \rightarrow \underline{\widetilde{D}}_{! * \mathbf{G}_{\mathbf{O}}}(\mathbf{G r} \times \mathbf{V})$ and its localization $\widetilde{\Phi}^{1,1}: D_{\text {perf }}^{G_{\overline{0}} \times \mathbb{C}^{\times}}\left(\mathfrak{G}_{1,1}^{\bullet}\right) \stackrel{\sim}{\longrightarrow} \widetilde{D}_{! * \mathbf{G}_{\mathbf{O}}}(\mathbf{G r} \times \mathbf{V})$.

We have the similar diagram of morphisms of functors $D_{\text {perf }}^{G_{\overline{0}} \times \mathbb{C}^{\times}}\left(\mathfrak{G}_{1,1}^{\bullet}\right) \rightarrow$ $\widetilde{D}_{! * \mathbf{G}_{\mathbf{O}}}(\mathbf{G r} \times \mathbf{V})$ with commutative upper and lower rectangles

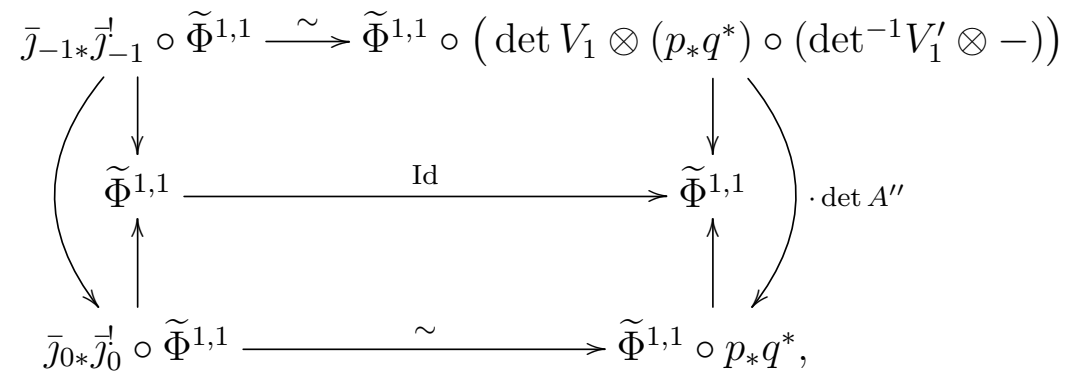

and we have to prove the commutativity of the big curved quadrangle. The endofunctors det $V_{1} \otimes\left(p_{*} q^{*}\right) \circ\left(\operatorname{det}^{-1} V_{1}^{\prime} \otimes-\right)$, Id, $p_{*} q^{*}$ of $D_{\text {perf }}^{G_{\overline{0}} \times \mathbb{C}^{\times}}\left(\mathfrak{G}_{1,1}^{\bullet}\right)$ are given by their respective kernels $K_{1}, K_{2}, K_{3}$ in $\underline{D}_{\text {perf }}^{G_{0}^{2} \times \mathbb{C}^{\times}}\left(\mathfrak{G}_{1,1}^{\bullet} \otimes \mathfrak{G}_{1,1}^{\bullet}\right)$. Note that the equivariance with respect to $\mathbb{C}^{\times}$(as opposed to $\left.\left(\mathbb{C}^{\times}\right)^{2}\right)$ suffices since all the three functors

\footnotetext{
${ }^{9}$ closed with respect to taking cones and direct summands
} 
under consideration commute with the shifts of the additional grading. Similarly, all the three functors on the constructible side commute with the Tate twists. All the three kernels are pure of weight 0, that is, their additional gradings coincide with their cohomological gradings. The category of pure weight 0 objects in $D_{\text {perf }}^{G_{0}^{2} \times \mathbb{C}^{\times}}\left(\mathfrak{G}_{1,1}^{\bullet} \otimes \mathfrak{G}_{1,1}^{\bullet}\right)$ is equivalent to the abelian category of $G_{\overline{0}}^{2} \times \mathbb{C}^{\times}$-equivariant $\mathfrak{G}_{1,1} \otimes \mathfrak{G}_{1,1}$-modules (the equivalence being obtained by taking cohomology). Therefore, the morphisms of functors (det $\left.V_{1} \otimes-\right) \circ\left(p_{*} q^{*}\right) \circ\left(\operatorname{det}^{-1} V_{1}^{\prime} \otimes-\right) \rightarrow \mathrm{Id}$ and $p_{*} q^{*} \rightarrow$ Id arise from the morphisms between the respective kernels that are injective as morphisms of $\mathfrak{G}_{1,1} \otimes \mathfrak{G}_{1,1}$-modules. We have to compare certain morphisms to $\widetilde{\Phi}^{1,1} K_{3}$, and we know that their compositions with the monomorphism $\underline{\Phi}^{1,1} K_{3} \rightarrow \underline{\Phi}^{1,1} K_{2}$ coincide, hence the desired equality of morphisms. This completes our second proof of (b).

(c) follows from the comparison of the distinguished triangles

$$
\left(\bar{\jmath}_{-1 *} \bar{\jmath}_{-1}^{!} \rightarrow \bar{\jmath}_{0 *} \stackrel{!}{\prime}_{0} \rightarrow \jmath_{0 *} \jmath_{0}^{!}\right) \circ \Phi^{1,1}
$$

and

$$
\Phi^{1,1} \circ\left(\operatorname{det} V_{1} \otimes\left(p_{*} q^{*}\right) \circ\left(\operatorname{det}^{-1} V_{1}^{\prime} \otimes-\right) \rightarrow p_{*} q^{*} \rightarrow p_{0 *} q_{0}^{*}\right) .
$$

The proposition is proved.

3.13. Some invariant theory. Let $U_{1}, U_{2}, U_{3}$ be vector spaces of dimensions $n_{1}, n_{2}, n_{3}$. The irreducible polynomial (resp. antipolynomial) representations of $\operatorname{GL}\left(U_{i}\right)$ are realized in the Schur spaces $\mathbb{S}_{\boldsymbol{\lambda}} U_{i}\left(\right.$ resp. $\left.\mathbb{S}_{\boldsymbol{\lambda}} U_{i}^{*}\right)$, where $\boldsymbol{\lambda}$ is a partition with $\ell(\boldsymbol{\lambda}) \leq n_{i}$. We will also write $\mathbb{S}_{\boldsymbol{\lambda}^{*}} U_{i}$ for $\mathbb{S}_{\boldsymbol{\lambda}} U_{i}^{*}$, where

$$
\boldsymbol{\lambda}^{*}=-w_{0} \boldsymbol{\lambda}=\left(-\lambda_{n_{i}},-\lambda_{n_{i}-1}, \ldots,-\lambda_{2},-\lambda_{1}\right)
$$

for $\boldsymbol{\lambda}=\left(\lambda_{1}, \ldots, \lambda_{n_{i}}\right)$. We also set $\mathbb{S}_{\boldsymbol{\lambda}} U_{i}=0=\mathbb{S}_{\boldsymbol{\lambda}^{*}} U_{i}$ for $\ell(\boldsymbol{\lambda})>n_{i}$. We denote by $\operatorname{Hom}_{\leq n_{2}}\left(U_{1}, U_{3}\right) \subset \operatorname{Hom}\left(U_{1}, U_{3}\right)$ the subvariety formed by all the homomorphisms of rank $\leq n_{2}$.

Lemma 3.13.1. The composition of homomorphisms induces the following isomorphisms of $\mathrm{GL}\left(U_{1}\right) \times \mathrm{GL}\left(U_{3}\right)$-modules:

(a) $\mathbb{C}\left[\operatorname{Hom}_{\leq n_{2}}\left(U_{1}, U_{3}\right)\right] \stackrel{\sim}{\longrightarrow}\left(\mathbb{C}\left[\operatorname{Hom}\left(U_{1}, U_{2}\right)\right] \otimes \mathbb{C}\left[\operatorname{Hom}\left(U_{2}, U_{3}\right)\right]\right)^{\mathrm{GL}\left(U_{2}\right)}$.

(b) In case $n_{1} \leq n_{2}$, for a partition $\boldsymbol{\nu}$,

$\mathbb{S}_{\boldsymbol{\nu}} U_{1} \otimes \mathbb{C}\left[\operatorname{Hom}\left(U_{1}, U_{3}\right)\right] \stackrel{\sim}{\longrightarrow}\left(\mathbb{C}\left[\operatorname{Hom}\left(U_{1}, U_{2}\right)\right] \otimes \mathbb{S}_{\boldsymbol{\nu}} U_{2} \otimes \mathbb{C}\left[\operatorname{Hom}\left(U_{2}, U_{3}\right)\right]\right)^{\mathrm{GL}\left(U_{2}\right)}$.

(c) In case $n_{3} \leq n_{2}$, for a partition $\boldsymbol{\nu}$,

$\mathbb{C}\left[\operatorname{Hom}\left(U_{1}, U_{3}\right)\right] \otimes \mathbb{S}_{\nu} U_{3}^{*} \stackrel{\sim}{\longrightarrow}\left(\mathbb{C}\left[\operatorname{Hom}\left(U_{1}, U_{2}\right)\right] \otimes \mathbb{S}_{\boldsymbol{\nu}} U_{2}^{*} \otimes \mathbb{C}\left[\operatorname{Hom}\left(U_{2}, U_{3}\right)\right]\right)^{\mathrm{GL}\left(U_{2}\right)}$.

Proof. (a) We have $\mathbb{C}\left[\operatorname{Hom}\left(U_{i}, U_{j}\right)\right]=\bigoplus_{m \geq 0} \operatorname{Sym}^{m}\left(U_{j}^{*} \otimes U_{i}\right)=\bigoplus_{\boldsymbol{\lambda}} \mathbb{S}_{\boldsymbol{\lambda}} U_{i} \otimes \mathbb{S}_{\boldsymbol{\lambda}^{*}} U_{j}$ as a $\operatorname{GL}\left(U_{i}\right) \times \mathrm{GL}\left(U_{j}\right)$-module. Also, $\mathbb{C}\left[\operatorname{Hom}_{\leq r}\left(U_{i}, U_{j}\right)\right]=\bigoplus_{\ell(\boldsymbol{\lambda}) \leq r} \mathbb{S}_{\boldsymbol{\lambda}} U_{i} \otimes \mathbb{S}_{\boldsymbol{\lambda}^{*}} U_{j}$ as a $\mathrm{GL}\left(U_{i}\right) \times \mathrm{GL}\left(U_{j}\right)$-module. Clearly, $\left(\mathbb{S}_{\boldsymbol{\lambda}^{*}} U_{2} \otimes \mathbb{S}_{\boldsymbol{\mu}} U_{2}\right)^{\mathrm{GL}\left(U_{2}\right)}=\mathbb{C}^{\delta_{\boldsymbol{\lambda} \mu}}$. So the two 
sides of (a) are isomorphic as $\operatorname{GL}\left(U_{1}\right) \times \mathrm{GL}\left(U_{3}\right)$-modules. On the other hand, the morphism in question is injective since the composition morphism $\operatorname{Hom}\left(U_{1}, U_{2}\right) \times$ $\operatorname{Hom}\left(U_{2}, U_{3}\right) \rightarrow \operatorname{Hom}_{\leq n_{2}}\left(U_{1}, U_{3}\right)$ is dominant. Hence the morphism in question is an isomorphism.

(b) We consider a copy $U_{2}^{\prime}$ of $U_{2}$, we tensor both sides of (b) with $\mathbb{S}_{\boldsymbol{\nu}^{*}} U_{2}^{\prime}$, and take direct sum over all partitions $\boldsymbol{\nu}$ with $\ell(\boldsymbol{\nu}) \leq n_{2}$. Then we have to prove that the morphism (induced by the composition of arrows of the $D_{4}$-quiver in (3.13.1))

$$
\begin{aligned}
& \gamma: \mathbb{C}\left[\operatorname{Hom}\left(U_{1}, U_{2}^{\prime}\right)\right] \otimes \mathbb{C}\left[\operatorname{Hom}\left(U_{1}, U_{3}\right)\right] \rightarrow \\
&\left(\mathbb{C}\left[\operatorname{Hom}\left(U_{1}, U_{2}\right)\right] \otimes \mathbb{C}\left[\operatorname{Hom}\left(U_{2}, U_{2}^{\prime}\right)\right] \otimes \mathbb{C}\left[\operatorname{Hom}\left(U_{2}, U_{3}\right)\right]\right)^{\mathrm{GL}\left(U_{2}\right)}
\end{aligned}
$$

is an isomorphism.

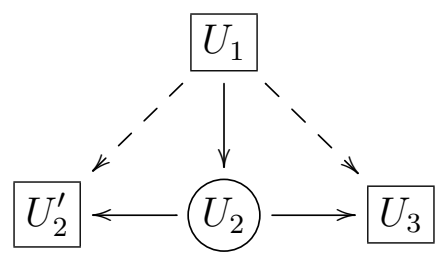

Now the statement can be reduced to (a) using the substitution $U_{3} \rightsquigarrow U_{3} \oplus U_{2}^{\prime}$. Alternatively, the condition $n_{1} \leq n_{2}$ guarantees that the morphism from the representation space of the $D_{4}$-quiver to the representation space of the dashed $A_{3}$-quiver is dominant. Hence $\gamma$ is injective. The surjectivity of $\gamma$ follows e.g. from [LP, Theorem 1].

(c) is dual to (b).

3.14. The monoidal property of $\Phi^{2,0}$. Recall the notation of Section 3.4. The monoidal structure $*$ on $D_{\text {perf }}^{G_{\overline{0}}}\left(\mathfrak{G}_{2,0}^{\bullet}\right)$ is defined via the kernel $\mathbb{C}\left[\mathcal{Q}^{A}\right]_{2,0}^{\bullet}$ : a $G_{Q^{-}}$

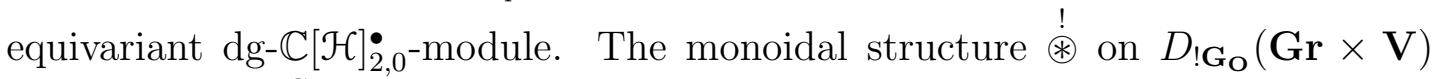
transfered to $D_{\text {perf }}^{G_{\overline{0}}}\left(\mathfrak{G}_{2,0}^{\bullet}\right)$ via the equivalence $\Phi^{2,0}$ is also defined via a kernel $\mathcal{K}^{\bullet}$ (a $G_{Q}$-equivariant dg- $\mathbb{C}[\mathcal{H}]_{2,0}^{\bullet}$-module). We have to construct an isomorphism of $G_{Q^{-}}$equivariant dg-C $[\mathcal{H}]_{2,0}$-modules $\mathbb{C}\left[Q^{A}\right]_{2,0} \stackrel{\sim}{\longrightarrow} \mathcal{K}^{\bullet}$.

We denote by $\mathbb{C}\left[\mathcal{H}_{\text {loc }}\right]_{2,0}^{\bullet}$ the localization of $\mathbb{C}[\mathcal{H}]_{2,0}^{\bullet}$ defined as $\mathbb{C}[\mathcal{H}]_{2,0}^{\bullet}\left[\operatorname{det}^{-1} A, \operatorname{det}^{-1} A^{\prime}, \operatorname{det}^{-1} A^{\prime \prime}\right] . \quad$ We define $\mathcal{K}_{\text {loc }}^{\bullet}:=\mathbb{C}\left[\mathcal{H}_{\text {loc }}\right]_{2,0} \otimes_{\mathbb{C}[\mathcal{H}]_{2,0}} \mathcal{K}^{\bullet}$ and $\mathbb{C}\left[Q_{\text {loc }}^{A}\right]_{2,0}^{\bullet}:=\mathbb{C}\left[\mathcal{H}_{\text {loc }}\right]_{2,0}^{\bullet} \otimes \mathbb{C}[\mathcal{H}]_{2,0} \mathbb{C}\left[\mathcal{Q}^{A}\right]_{2,0}$.

We have $\Phi^{2,0} \mathfrak{G}_{2,0}^{\bullet} \simeq \varrho_{\text {right }}^{-1} E_{0}$. Also, for $\mathcal{F} \in D_{! \mathbf{G}_{\mathbf{O}}}(\mathbf{G r} \times \mathbf{V})$ we have $\varrho_{\text {right }}^{-1} E_{0} \stackrel{!}{\circledast} \mathcal{F}=\bar{\jmath}_{0 *} \bar{J}_{0}^{1} \mathcal{F}$. Thus Proposition 3.12.1(a) yields an isomorphism of functors $\Phi^{2,0}\left(\mathfrak{G}_{2,0}^{\bullet} \quad \stackrel{*}{*}-\right) \stackrel{\sim}{\longrightarrow}\left(\Phi^{2,0} \mathfrak{G}_{2,0}^{\bullet}\right) \stackrel{!}{*} \Phi^{2,0}-$. This isomorphism yields in turn an isomorphism of kernels $\mathbb{C}\left[Q_{\text {forg }}^{A}\right]_{2,0}^{\bullet} \stackrel{\sim}{\longrightarrow} \mathcal{K}_{\text {forg }}^{\bullet}$, where the subscript forg denotes the restriction of the dg-module structure from 
$\mathbb{C}[\mathcal{H}]_{2,0}^{\bullet}$ to $\mathbb{C}\left[\mathcal{H}_{\text {forg }}\right]_{2,0}^{\bullet}:=\operatorname{Sym}\left(\operatorname{Hom}\left(V_{1}, V_{2}\right)[-2]\right) \otimes \operatorname{Sym}\left(\operatorname{Hom}\left(V_{2}, V_{1}\right)\right) \otimes$ $\operatorname{Sym}\left(\operatorname{Hom}\left(V_{1}^{\prime}, V_{2}\right)[-2]\right) \otimes \operatorname{Sym}\left(\operatorname{Hom}\left(V_{2}, V_{1}^{\prime}\right)\right)$.

According to Proposition 3.12.1(b), the following diagram of functors $D_{\text {perf }}^{G_{\overline{0}}}\left(\mathfrak{G}_{2,0}^{\bullet}\right) \rightarrow D_{! \mathbf{G}_{\mathbf{O}}}(\mathbf{G r} \times \mathbf{V})$ commutes:

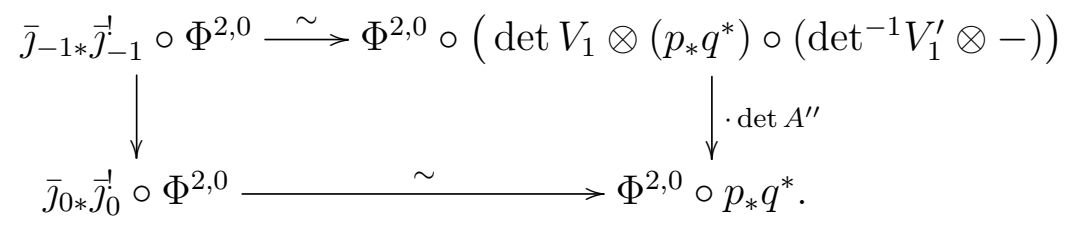

Hence the diagram

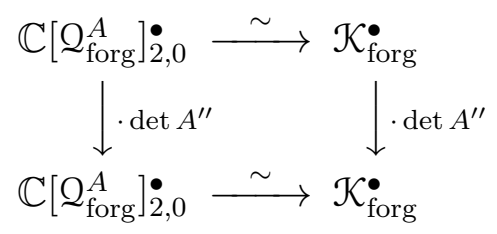

commutes as well, and in particular the multiplication by $\operatorname{det} A^{\prime \prime}$ is injective on $\mathcal{K}^{\bullet}$, and hence $\mathcal{K}^{\bullet} \hookrightarrow \mathcal{K}_{\text {loc }}^{\bullet}$

Now since $\Phi_{\text {loc }}^{2,0}: D_{\text {perf }}^{G_{\overline{0}}}\left(\mathfrak{A}^{\bullet}\right) \stackrel{\sim}{\longrightarrow} D_{\mathbf{G}_{\mathbf{O}}}(\mathbf{G r}) \cong D_{! \mathbf{G}_{\mathbf{O}}}^{\text {loc }}(\mathbf{G r} \times \mathbf{V})$ (see Lemma 3.10 .1 and Corollary 3.10.2) coincides with the equivariant Satake equivalence, and the latter one is monoidal, we obtain an isomorphism of localized kernels $\mathbb{C}\left[Q_{\text {loc }}^{A}\right]_{2,0}^{\bullet} \stackrel{\sim}{\longrightarrow}$ $\mathcal{K}_{\text {loc }}^{\bullet}$ as $G_{Q}$-equivariant $\mathbb{C}\left[\mathcal{H}_{\text {loc }}\right]_{2,0}^{\bullet}$-modules. By the argument in the second proof of Proposition 3.12.1(b) (using the additional grading and purity of $\mathcal{K}^{\bullet}$ ), it remains to verify that this isomorphism restricts to the desired isomorphism from $\mathbb{C}\left[Q^{A}\right]_{2,0}^{\bullet} \subset \mathbb{C}\left[Q_{\text {loc }}^{A}\right]_{2,0}^{\bullet}$ to $\mathcal{K}^{\bullet} \subset \mathcal{K}_{\text {loc }}^{\bullet}$. For this verification it suffices to restrict the scalars to $\mathbb{C}\left[\mathcal{H}_{\text {forg }}\right]_{2,0}^{\bullet}$. But we have already seen that over $\mathbb{C}\left[\mathcal{H}_{\text {forg }}\right]_{2,0}^{\bullet}$ we obtain an isomorphism $\mathbb{C}\left[Q_{\text {forg }}^{A}\right]_{2,0}^{\bullet} \stackrel{\sim}{\longrightarrow} \mathcal{K}_{\text {forg }}^{\bullet}$.

This completes the proof of the monoidal property of $\Phi^{2,0}$.

3.15. Fourier Transform. We have the Fourier transform functors (along V) $\mathrm{FT}: D_{! \mathbf{G}_{\mathbf{O}}}(\mathbf{G r} \times \mathbf{V}) \rightarrow D_{* \mathbf{G}_{\mathbf{O}}}(\mathbf{G r} \times \mathbf{V}), \mathrm{FT}: D_{* \mathbf{G}_{\mathbf{O}}}(\mathbf{G r} \times \mathbf{V}) \rightarrow D_{! \mathbf{G}_{\mathbf{O}}}(\mathbf{G r} \times \mathbf{V})$, FT: $D_{! * \mathbf{G}_{\mathbf{O}}}(\mathbf{G r} \times \mathbf{V}) \rightarrow D_{! * \mathbf{G}_{\mathbf{O}}}(\mathbf{G r} \times \mathbf{V})$.

Strictly speaking, the Fourier transform goes not to $D_{? \mathbf{G}_{\mathbf{O}}}(\mathbf{G r} \times \mathbf{V})$, but to $D_{? \mathbf{G}_{\mathbf{O}}}\left(\mathbf{G r} \times\left(V^{*} \otimes \mathbf{F}\right)\right)$. However, we identify $V^{*}$ with $V$ using our choice of (selfdual) basis $e_{1}, \ldots, e_{N}$, and accordingly change the action of $\mathbf{G}_{\mathbf{O}}=\mathrm{GL}(N, \mathbf{O})$ by composing it with the automorphism $g \mapsto{ }^{t} g^{-1}$. Note that the resulting Fourier transform to $D_{? \mathbf{G}_{\mathbf{O}}}(\mathbf{G r} \times \mathbf{V})$ is independent of the choice of basis in $V$.

To describe the effect of FT on the coherent side, we identify $V_{1} \cong V_{1}^{*}$ and $V_{2} \cong V_{2}^{*}$ using our bases. Furthermore, we identify

$$
\operatorname{Hom}\left(V_{1}, V_{2}\right) \cong \operatorname{Hom}\left(V_{2}^{*}, V_{1}^{*}\right) \cong \operatorname{Hom}\left(V_{2}, V_{1}\right), A \mapsto B:=-{ }^{t} A,
$$




$$
\operatorname{Hom}\left(V_{2}, V_{1}\right) \cong \operatorname{Hom}\left(V_{1}^{*}, V_{2}^{*}\right) \cong \operatorname{Hom}\left(V_{1}, V_{2}\right), B \mapsto A:={ }^{t} B .
$$

Thus we obtain an $\iota$-equivariant transposition isomorphism

$$
\begin{aligned}
\tau: \mathfrak{G}_{2,0}^{\bullet}=\operatorname{Sym}\left(\operatorname{Hom}\left(V_{1}, V_{2}\right)\right) \otimes \operatorname{Sym}\left(\operatorname{Hom}\left(V_{2}, V_{1}\right)[-2]\right) \\
\rightarrow \operatorname{Sym}\left(\operatorname{Hom}\left(V_{1}, V_{2}\right)[-2]\right) \otimes \operatorname{Sym}\left(\operatorname{Hom}\left(V_{2}, V_{1}\right)\right)=\mathfrak{G}_{0,2}^{\bullet},
\end{aligned}
$$

where $\iota: G_{\overline{0}} \rightarrow G_{\overline{0}}$ is an automorphism $\left(g_{1}, g_{2}\right) \mapsto\left({ }^{t} g_{1}^{-1},{ }^{t} g_{2}^{-1}\right)$. We denote the extension of scalars via $\tau$ by $\varphi \tau: D_{\text {perf }}^{G_{\overline{0}}}\left(\mathfrak{G}_{2,0}^{\bullet}\right) \rightarrow D_{\text {perf }}^{G_{\overline{0}}}\left(\mathfrak{G}_{0,2}^{\bullet}\right)$. Clearly, the functor $\varphi \tau:\left(D_{\text {perf }}^{G_{\overline{0}}}\left(\mathfrak{G}_{2,0}^{\bullet}\right), \stackrel{*}{*}\right) \rightarrow\left(D_{\text {perf }}^{G_{\overline{0}}}\left(\mathfrak{G}_{0,2}^{\bullet}\right), \begin{array}{l}B \\ *\end{array}\right)$ is monoidal. Also, by the standard properties of the Fourier transform, the functor

$$
\text { FT: }\left(D_{! \mathbf{G}_{\mathbf{O}}}(\mathbf{G r} \times \mathbf{V}), \stackrel{!}{\circledast}\right) \rightarrow\left(D_{* \mathbf{G}_{\mathbf{O}}}(\mathbf{G r} \times \mathbf{V}), \stackrel{*}{*}\right)
$$

is monoidal. Thus the monoidal property of $\Phi^{0,2}$ is a corollary of the following

Proposition 3.15.1. The functors $\mathrm{FT} \circ \Phi^{2,0}: D_{\text {perf }}^{G_{\overline{0}}}\left(\mathfrak{G}_{2,0}^{\bullet}\right) \rightarrow D_{* \mathbf{G}_{\mathbf{O}}}(\mathbf{G r} \times \mathbf{V})$ and $\Phi^{0,2} \circ \varphi \tau$ are isomorphic.

Proof. Going over the construction of equivalences of $\Phi^{0,2}, \Phi^{2,0}$, we see that it suffices to construct the isomorphisms FT( $\left.E_{0}\right) \stackrel{\sim}{\longrightarrow} E_{0}$ (evident), and

$$
\mathrm{FT}\left(-_{\text {left }} * \bullet *-_{\text {right }}\right) \stackrel{\sim}{\sim} \iota\left(-_{\text {left }}\right) * \mathrm{FT}(\bullet) * \iota\left(-_{\text {right }}\right)
$$

(left and right convolution functors

$$
\left.D_{\mathbf{G}_{\mathbf{O}}}(\mathbf{G r}) \times D_{! * \mathbf{G}_{\mathbf{O}}}(\mathbf{G r} \times V) \times D_{\mathbf{G}_{\mathbf{O}}}(\mathbf{G r}) \rightarrow D_{! * \mathbf{G}_{\mathbf{O}}}(\mathbf{G r} \times V)\right) .
$$

Here $\iota: D_{\mathbf{G}_{\mathbf{O}}}(\mathbf{G r}) \rightarrow D_{\mathbf{G}_{\mathbf{O}}}(\mathbf{G r})$ is a monoidal autoequivalence induced by the automorphism $\iota: g \mapsto{ }^{t} g^{-1}$ of $\mathbf{G}_{\mathbf{F}}$. Note that the Satake equivalence intertwines $\iota: D_{\mathbf{G}_{\mathbf{O}}}(\mathbf{G r}) \rightarrow D_{\mathbf{G}_{\mathbf{O}}}(\mathbf{G r})$ with the same named autoequivalence of $\operatorname{Rep}\left(\mathrm{GL}_{N}\right)$. Also note that $\iota: D_{\mathbf{G}_{\mathbf{O}}}(\mathbf{G r}) \rightarrow D_{\mathbf{G}_{\mathbf{O}}}(\mathbf{G r})$ is induced by the automorphism $L \mapsto$ $L^{\perp}$ of $\mathbf{G r}$. Here $L^{\perp}:=\{v \in \mathbf{V}:(v, L) \in \mathbf{O}\}$, and $($, ) stands for the $\mathbf{F}$-bilinear pairing on $\mathbf{V}$ such that $\left(e_{i}, e_{j}\right)=\delta_{i j}$. Now the existence of the desired isomorphism (3.15.1) follows from the definitions of FT and convolutions (3.2.3, 3.2.4).

3.16. The monoidal property of $\Phi^{1,1}$. The argument is very similar to the one of Section 3.14. We introduce

$$
\begin{aligned}
& \mathbb{C}[\mathcal{H}]_{1,1}^{\bullet}=\operatorname{Sym}\left(\operatorname{Hom}\left(V_{1}, V_{2}\right)[-1]\right) \otimes \operatorname{Sym}\left(\operatorname{Hom}\left(V_{2}, V_{1}\right)[-1]\right) \otimes \operatorname{Sym}\left(\operatorname{Hom}\left(V_{1}^{\prime}, V_{2}\right)[-1]\right) \\
& \otimes \operatorname{Sym}\left(\operatorname{Hom}\left(V_{2}, V_{1}^{\prime}\right)[-1]\right) \otimes \operatorname{Sym}\left(\operatorname{Hom}\left(V_{1}, V_{1}^{\prime}\right)[-1]\right) \otimes \operatorname{Sym}\left(\operatorname{Hom}\left(V_{1}^{\prime}, V_{1}\right)[-1]\right) .
\end{aligned}
$$

Then the monoidal structure $\otimes_{\mathfrak{G}_{i, 1}}$ on $D_{\text {perf }}^{G_{\overline{0}}}\left(\mathfrak{G}_{1,1}^{\bullet}\right)$ is defined via the kernel $\mathbb{C}[\Delta]_{1,1}^{\bullet}$ :

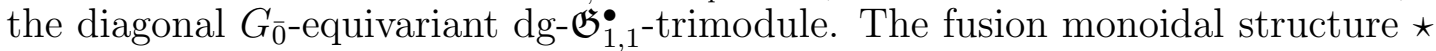
on $D_{! * \mathbf{G}_{\mathbf{O}}}(\mathbf{G r} \times \mathbf{V})$ transferred to $D_{\text {perf }}^{G_{\overline{0}}}\left(\mathfrak{G}_{1,1}^{\bullet}\right)$ via the equivalence $\Phi^{1,1}$ is also defined via a kernel $\mathrm{K}^{\bullet}$ (a $G_{\overline{0}}$-equivariant $\mathrm{dg}-\mathfrak{G}_{1,1}^{\bullet}$-trimodule). Note that we have a 
different equivariant structure than for $\mathcal{K}^{\bullet}$ (notation of $\S 3.14$ ) because of the different compatibility with the Hecke action. We have to construct an isomorphism of $G_{\overline{0}}$-equivariant dg- $\mathfrak{G}_{1,1}$-trimodules $\mathbb{C}[\Delta]_{1,1} \stackrel{\sim}{\sim} \mathrm{K}^{\bullet}$.

We have $\Phi^{1,1} \mathfrak{G}_{1,1}^{\bullet} \simeq E_{0}$. Also, we have an isomorphism of endofunctors $E_{0} \star-\cong$ Id $: D_{! * \mathbf{G}_{\mathbf{O}}}(\mathbf{G r} \times \mathbf{V}) \rightarrow D_{! * \mathbf{G}_{\mathbf{O}}}(\mathbf{G r} \times \mathbf{V})$. Thus we obtain an isomorphism of functors $\Phi^{1,1}\left(\mathfrak{G}_{1,1}^{\bullet} \otimes_{\mathfrak{G}_{1,1}^{\bullet}}-\right) \stackrel{\sim}{\sim}\left(\Phi^{1,1} \mathfrak{G}_{1,1}^{\bullet}\right) \star \Phi^{1,1}-$. This isomorphism yields an isomorphism of kernels $\mathbb{C}\left[\Delta_{\text {forg }}\right]_{1,1}^{\bullet} \stackrel{\sim}{\longrightarrow} \mathrm{K}_{\text {forg }}^{\bullet}$ (notation is explained in Section 3.14).

The following diagram commutes:

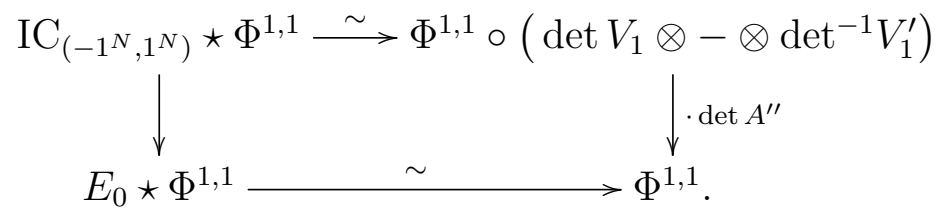

Hence the diagram

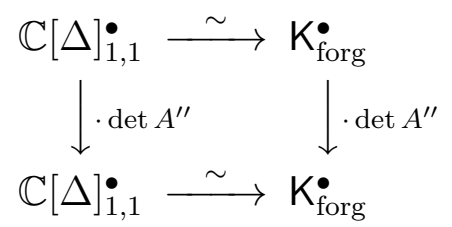

commutes as well, and the rest of the argument proceeds just as in Section 3.14.

This completes the proof of the monoidal property of $\Phi^{1,1}$ along with Theorem 3.6.1.

\section{A COherent Realization of $D_{\mathrm{GL}(N-1, \mathbf{O})}(\mathbf{G r})$}

4.1. Notation. We consider a complex vector space $\bar{V}_{1}$ with a basis $e_{2}, e_{3}, \ldots, e_{N}$. We consider the Lie superalgebra $\mathfrak{g l}(N-1 \mid N)=\mathfrak{g l}\left(\bar{V}_{1} \oplus \Pi V_{2}\right)$. We have a decomposition $\mathfrak{g l}(N-1 \mid N)=\overline{\mathfrak{g}}_{\overline{0}} \oplus \overline{\mathfrak{g}}_{\overline{1}}$, where $\overline{\mathfrak{g}}_{\overline{1}}=\Pi \operatorname{Hom}\left(\bar{V}_{1}, V_{2}\right) \oplus \Pi \operatorname{Hom}\left(V_{2}, \bar{V}_{1}\right)$, and $\overline{\mathfrak{g}}_{\overline{0}}=\operatorname{End}\left(\bar{V}_{1}\right) \oplus \operatorname{End}\left(V_{2}\right)$. We set $\bar{G}_{\overline{0}}=\operatorname{GL}\left(\bar{V}_{1}\right) \times \operatorname{GL}\left(V_{2}\right)$. We consider the dg-algebra $\overline{\mathfrak{G}}^{\bullet}=\operatorname{Sym}\left(\overline{\mathfrak{g}}_{\overline{1}}[-1]\right)$ with trivial differential, and the triangulated category $D_{\text {perf }}^{\bar{G}_{\overline{0}}}\left(\overline{\mathfrak{G}}^{\bullet}\right)$ obtained by localization (with respect to quasi-isomorphisms) of the category of perfect $\bar{G}_{\overline{0}}$-equivariant $\mathrm{dg}$ - $\overline{\mathfrak{G}}{ }^{\bullet}$-modules. Finally, we set $\overline{\mathbf{G}}_{\mathbf{O}}=$ $\mathrm{GL}(N-1, \mathrm{O})$.

Theorem 4.1.1. There exists an equivalence of triangulated categories $\bar{\Phi}: D_{\text {perf }}^{\bar{G}_{\overline{0}}}\left(\overline{\mathfrak{G}}^{\bullet}\right) \stackrel{\sim}{\longrightarrow} D_{\overline{\mathbf{G}}_{\mathbf{O}}}(\mathbf{G r})$ commuting with the left convolution action of the monoidal spherical Hecke category $\operatorname{Perv}_{\mathrm{GL}(N-1, \mathbf{O})}\left(\mathbf{G r}_{N-1}\right) \cong \operatorname{Rep}\left(\mathrm{GL}_{N-1}\right)$ and with the right convolution action of the monoidal spherical Hecke category $\operatorname{Perv}_{\mathrm{GL}(N, \mathbf{O})}\left(\mathbf{G r}_{N}\right) \cong \operatorname{Rep}\left(\mathrm{GL}_{N}\right)$.

The proof will be given in Section 4.4 after some preparations in $\S \S 4.2,4.3$. 
Similarly to Section 3.8, we define $\bar{\Lambda}:=\Lambda\left(\operatorname{Hom}\left(\bar{V}_{1}, V_{2}\right) \oplus \operatorname{Hom}\left(V_{2}, \bar{V}_{1}\right)\right)$. We consider the derived category $D_{\mathrm{fd}}^{\bar{G}_{\overline{0}}}(\bar{\Lambda})$ of finite dimensional complexes of $\bar{G}_{\overline{0}} \ltimes \bar{\Lambda}$ modules. We have the Koszul equivalence functors

$$
\bar{\varkappa}: D_{\mathrm{fd}}^{\bar{G}_{\overline{0}}}(\bar{\Lambda}) \stackrel{\sim}{\longrightarrow} D_{\text {perf }}^{\bar{G}_{\overline{0}}}\left(\overline{\mathfrak{G}}^{\bullet}\right), S D_{\mathrm{fd}}^{\bar{G}_{\overline{0}}}(\bar{\Lambda}) \stackrel{\sim}{\longrightarrow} S D_{\text {perf }}^{\bar{G}_{\overline{0}}}\left(\overline{\mathfrak{G}}^{\bullet}\right) .
$$

We also consider the degeneration $\mathfrak{g l}(N-1 \mid N)$ of the Lie superalgebra $\mathfrak{g l}(N-1 \mid N)$ (defined as in $\S 1.5$ ), and the derived category of bounded complexes of integrable $\mathfrak{g l}(N-1 \mid N)$-modules $S D_{\text {int }}(\mathfrak{g l}(N-1 \mid N)) \cong S D_{\mathrm{fd}}^{\bar{G}_{\overline{0}}}(\bar{\Lambda})$. The following corollary of Theorem 4.1.1 is proved just like Corollary 3.8.1.

Corollary 4.1.2. (a) The composed equivalence

$$
\bar{\Phi} \circ \bar{\varkappa}: D_{\mathrm{fd}}^{\bar{G}_{\overline{0}}}(\bar{\Lambda}) \stackrel{\sim}{\longrightarrow} D_{\overline{\mathbf{G}}_{\mathbf{O}}}(\mathbf{G r})
$$

is exact with respect to the tautological t-structure on $D_{\mathrm{fd}}^{\bar{G}_{\bar{D}}}(\bar{\Lambda})$ and the perverse t-structure on $D_{\overline{\mathbf{G}}_{\mathbf{O}}}(\mathbf{G r})$.

(b) This equivalence is monoidal with respect to the tensor structure on $D_{\mathrm{fd}}^{\bar{G}_{\overline{0}}}(\bar{\Lambda})$ and the fusion $\star$ on $D_{\overline{\mathbf{G}}_{\mathbf{O}}}(\mathbf{G r})$.

(c) The equivariant derived category $D_{\overline{\mathbf{G}}_{\mathbf{O}}}(\mathbf{G r})$ is equivalent to the bounded derived category of the abelian category $\operatorname{Perv}_{\overline{\mathbf{G}}_{\mathbf{O}}}(\mathbf{G r})$.

In case $N=2$, both Theorem 4.1.1 and Corollary 4.1.2(a) were proved in [BrF] by a rather different argument.

4.2. Constructible mirabolic restriction. Clearly, $\mathbf{V}_{0} \backslash t \mathbf{V}_{0}$ is a single $\mathbf{G}_{\mathbf{O}^{-}}$ orbit, and the stabilizer of the vector $e_{1} \in \mathbf{V}_{0} \backslash t \mathbf{V}_{0}$ is the mirabolic subgroup $\mathbf{M}_{\mathbf{O}} \subset \mathbf{G}_{\mathbf{O}}$. Hence $D_{\mathbf{G}_{\mathbf{O}}}\left(\mathbf{G r} \times\left(\mathbf{V}_{0} \backslash t \mathbf{V}_{0}\right)\right) \cong D_{\mathbf{M}_{\mathbf{O}}}(\mathbf{G r})$. We will denote $\mathbf{G r}=$ $\mathbf{G r}_{\mathrm{GL}_{N}}$ by $\mathbf{G r}_{N}$ to distinguish it from $\mathbf{G r}_{N-1}:=\mathbf{G r}_{\mathrm{GL}_{N-1}}$. We will also denote by $\mathbf{G r}_{N}^{\subset} \subset \mathbf{G r}_{N}$ (resp. $\mathbf{G r}_{N-1}^{\subset} \subset \mathbf{G r}_{N-1}$ ) the closed subvariety classifying the sublattices in the standard one $\mathbf{V}_{0}$ (resp. in $\overline{\mathbf{V}}_{0}:=\mathbf{O} e_{2} \oplus \mathbf{O} e_{3} \oplus \ldots \oplus \mathbf{O} e_{N}$ ). It is a union of the Schubert varieties numbered by the negative partitions of length $\leq N$ $($ resp. $\leq N-1)$. The category $\operatorname{Perv}_{\mathrm{GL}(N, \mathbf{O})}\left(\mathbf{G r}_{N}^{\subset}\right)\left(\operatorname{resp} . \operatorname{Perv}_{\mathrm{GL}(N-1, \mathbf{O})}\left(\mathbf{G r}_{N-1}^{\complement}\right)\right)$ is monoidal and the Satake equivalence takes it to the monoidal category of polynomial representations of $\mathrm{GL}_{N}$ (resp. $\mathrm{GL}_{N-1}$ ).

We have a closed embedding $\varsigma: \mathbf{G r}_{N-1}^{\subset} \hookrightarrow \mathbf{G r}_{N}^{\subset},\left(\bar{L} \subset \overline{\mathbf{V}}_{0}\right) \mapsto\left(\mathbf{O} e_{1} \oplus \bar{L} \subset \mathbf{V}_{0}\right)$.

Lemma 4.2.1. (a) The functor $\varsigma^{!}: D_{\mathrm{GL}(N, \mathbf{O})}\left(\mathbf{G r}_{N}^{\subset}\right) \rightarrow D_{\mathrm{GL}(N-1, \mathbf{O})}\left(\mathbf{G r}_{N-1}^{\subset}\right)$ is monoidal.

(b) For a negative partition $\boldsymbol{\lambda}$ we have $\varsigma$ ! $\mathrm{IC}_{\boldsymbol{\lambda}}=\mathrm{IC}_{\boldsymbol{\lambda}}[|\boldsymbol{\lambda}|]$ in notation of Section 3.13 (i.e. if $\lambda_{1}<0$, then $\varsigma^{!} \mathrm{IC}_{\boldsymbol{\lambda}}=0$, and if $\lambda_{1}=0$, then $\left.\varsigma ! \mathrm{IC}_{\boldsymbol{\lambda}}=\mathrm{IC}_{\left(\lambda_{2} \geq \ldots \geq \lambda_{N}\right)}\left[\lambda_{2}+\ldots+\lambda_{N}\right]\right)$. 
Proof. (a) The Grassmannian of sublattices $\mathbf{G r}_{N}^{\subset}$ (resp. $\mathbf{G r}_{N-1}^{\subset}$ ) is the union of connected components $\bigsqcup_{n \in \mathbb{N}} \mathbf{G r}_{N}^{\subset,(-n)}$ (resp. $\bigsqcup_{n \in \mathbb{N}} \mathbf{G r}_{N-1}^{\subset,(-n)}$ ) parametrizing sublattices of codimension $n$. Clearly, $\varsigma\left(\mathbf{G r}_{N-1}^{\subset,(-n)}\right) \subset \mathbf{G r}_{N}^{\subset,(-n)}$. Moreover, $\varsigma\left(\mathbf{G r}_{N-1}^{\subset,(-n)}\right)$ is a connected component of the intersection of the Levi Grassmannian $\mathbf{G r}_{\mathrm{GL}_{1} \times \mathrm{GL}_{N-1}} \subset \mathbf{G r}_{N}$ with $\mathbf{G r}_{N}^{\subset,(-n)}$. We have the monoidal functor of hyperbolic restriction to the Levi Grassmannian, see e.g. [BD, 5.3.28 at page 213]. Since $\left(\mathbf{M}_{\mathbf{F}} \cdot \varsigma\left(\mathbf{G r}_{N-1}^{\subset,(-n)}\right)\right) \cap \mathbf{G r}_{N}^{\subset,(-n)}=\varsigma\left(\mathbf{G r}_{N-1}^{\subset,(-n)}\right)$, the hyperbolic restriction to the component $\varsigma\left(\mathbf{G r}_{N-1}^{\subset,(-n)}\right)$ coincides with the corestriction $\varsigma$ !

(b) We have $\left(\mathbb{S}_{\lambda} V_{1}^{*}\right)^{\mathrm{GL}_{1}}=\mathbb{S}_{\boldsymbol{\lambda}} \bar{V}_{1}^{*}$. Now the desired claim follows from [BD, Proposition 5.3.29].

Recall that the monoidal Hecke category $D_{\mathrm{GL}(N, \mathbf{O})}\left(\mathbf{G r}_{N}\right)=D_{\mathbf{G}_{\mathbf{O}}}(\mathbf{G r})$ acts by the left convolution on $D_{! * \mathbf{G}_{\mathbf{O}}}(\mathbf{G r} \times \mathbf{V})$. In particular, the monoidal subcategory $D_{\mathbf{G}_{\mathbf{O}}}\left(\mathbf{G r}_{N}^{\subset}\right) \subset D_{\mathbf{G}_{\mathbf{O}}}(\mathbf{G r})$ acts on $D_{! * \mathbf{G}_{\mathbf{O}}}(\mathbf{G r} \times \mathbf{V})$. We define the action of $D_{\mathbf{G}_{\mathbf{O}}}\left(\mathbf{G r}_{N}^{\subset}\right)$ by the left convolution on $D_{\mathbf{G}_{\mathbf{O}}}\left(\mathbf{G r} \times\left(\mathbf{V}_{0} \backslash t \mathbf{V}_{0}\right)\right)$ as follows: $\mathcal{A} * \mathcal{F}:=\jmath_{0}^{!}\left(\mathcal{A} * \jmath_{0 *} \mathcal{F}\right)$ (notation of Section 3.12). Also, the monoidal Hecke category $D_{\mathrm{GL}(N-1, \mathbf{O})}\left(\mathbf{G r}_{N-1}\right)$ acts by the left convolution on $D_{\mathrm{GL}(N-1, \mathbf{O})}\left(\mathbf{G r}_{N}\right)$. Finally, we denote by $\operatorname{Res}_{\mathbf{M}_{\mathbf{O}}}^{\mathrm{GL}(N-1, \mathbf{O})}: D_{\mathbf{M}_{\mathbf{O}}}\left(\mathbf{G r}_{N}\right) \rightarrow D_{\mathrm{GL}(N-1, \mathbf{O})}\left(\mathbf{G r}_{N}\right)$ the functor of restriction of equivariance under $\mathrm{GL}(N-1, \mathbf{O}) \hookrightarrow \mathbf{M}_{\mathbf{O}}$.

Lemma 4.2.2. We have a compatible system of isomorphisms for all $\mathcal{A} \in D_{\mathrm{GL}(N, \mathbf{O})}\left(\mathbf{G r}_{N}^{\subset}\right)$ and $\mathcal{F} \in D_{\mathbf{G}_{\mathbf{O}}}\left(\mathbf{G r} \times\left(\mathbf{V}_{0} \backslash t \mathbf{V}_{0}\right)\right) \cong D_{\mathbf{M}_{\mathbf{O}}}(\mathbf{G r})$ :

$$
\operatorname{Res}_{\mathbf{M}_{\mathbf{O}}}^{\mathrm{GL}(N-1, \mathbf{O})}(\mathcal{A} * \mathcal{F}) \stackrel{\sim}{\longrightarrow} \varsigma^{!} \mathcal{A} * \operatorname{Res}_{\mathbf{M}_{\mathbf{O}}}^{\mathrm{GL}(N-1, \mathbf{O})}(\mathcal{F})
$$

Proof. Comparison of definitions.

4.3. Coherent mirabolic restriction. Recall the subcategory $D_{\text {perf }}^{G_{\overline{0}}, \geq}\left(\mathfrak{G}_{1,1}^{\bullet}\right) \subset$ $D_{\text {perf }}^{G_{\overline{0}}}\left(\mathfrak{G}_{1,1}^{\bullet}\right)$ generated by $\left\{V_{1, \boldsymbol{\lambda}} \otimes \mathfrak{G}_{1,1}^{\bullet} \otimes V_{2, \boldsymbol{\mu}} \mid \boldsymbol{\lambda} \geq 0\right\}$ introduced in the proof of Proposition 3.12.1(a). The equivalence $\Phi^{1,1}: D_{\text {perf }}^{G_{\overline{0}}}\left(\mathfrak{G}_{1,1}^{\bullet}\right) \stackrel{\sim}{\longrightarrow} D_{! * \mathbf{G}_{\mathbf{O}}}(\mathbf{G r} \times \mathbf{V})$ restricts to the same named equivalence $D_{\text {perf }}^{G_{\overline{0}}, \geq}\left(\mathfrak{G}_{1,1}^{\bullet}\right) \stackrel{\sim}{\longrightarrow} D_{! * \mathbf{G}_{\mathbf{O}}}\left(\mathbf{G r} \times \mathbf{V}_{0}\right)$, and the functor $p_{*} q^{*}: D_{\text {perf }}^{G_{\overline{0}}, \geq}\left(\mathfrak{G}_{1,1}^{\bullet}\right) \rightarrow D_{\text {perf }}^{G_{\overline{0}}, \geq}\left(\mathfrak{G}_{1,1}^{\bullet}\right)$ (notation of Section 3.12) is isomorphic to Id. Thus we have a natural morphism Id $\cong p_{*} q^{*} \rightarrow p_{0 *} q_{0}^{*}$ (notation of Section 3.12) of endofunctors of $D_{\text {perf }}^{G_{\overline{0}}, \geq}\left(\mathfrak{G}_{1,1}^{\bullet}\right)$. Composing with another copy of $p_{0 *} q_{0}^{*}$ we obtain a morphism $p_{0 *} q_{0}^{*} \rightarrow p_{0 *} q_{0}^{*} \circ p_{0 *} q_{0}^{*}$ of endofunctors of $D_{\text {perf }}^{G_{\overline{0}}, \geq}\left(\mathfrak{G}_{1,1}^{\bullet}\right)$ that is easily seen to be an isomorphism. Indeed, according to Proposition 3.12.1(c), $\Phi^{1,1}$ takes $p_{0 *} q_{0}^{*}$ to $\jmath_{0 *} \jmath_{0}^{!}$, and $\jmath_{0 *} \jmath_{0}^{!} \stackrel{\sim}{\longrightarrow} \jmath_{0 *} \jmath_{0} \circ \jmath_{0 *} \jmath_{0}$. Inverting the isomorphism $p_{0 *} q_{0}^{*} \stackrel{\sim}{\longrightarrow} p_{0 *} q_{0}^{*} \circ p_{0 *} q_{0}^{*}$, we obtain an isomorphism $p_{0 *} q_{0}^{*} \circ p_{0 *} q_{0}^{*} \stackrel{\sim}{\longrightarrow} p_{0 *} q_{0}^{*}$ that, together with the morphism Id $\rightarrow p_{0 *} q_{0}^{*}$ equips $p_{0 *} q_{0}^{*}$ with a structure of (idempotent) monad in $D_{\text {perf }}^{G_{\overline{0}}, \geq}\left(\mathfrak{G}_{1,1}^{\bullet}\right)$. 
We denote the dg-algebra with trivial differential $\mathfrak{G}_{1,1}^{\bullet} \otimes \mathbb{C}\left[\operatorname{Hom}_{\leq N-1}\left(V_{1}, V_{1}^{\prime}\right)\right]$ (the grading on $\mathbb{C}\left[\operatorname{Hom}_{\leq N-1}\left(V_{1}, V_{1}^{\prime}\right)\right]$ is trivial) by $\mathfrak{F}^{\bullet}$ (here $\operatorname{Hom}_{\leq N-1}\left(V_{1}, V_{1}^{\prime}\right) \subset$ $\operatorname{Hom}\left(V_{1}, V_{1}^{\prime}\right)$ stands for the subvariety formed by the noninvertible homomorphisms). It is acted upon by $G_{Q}$. By Theorem 3.6.1 and Proposition 3.12.1, we have an equivalence of categories $\Phi^{\prime}$ from the Kleisli category (see [M, VI.5]) $D\left(p_{0 *} q_{0}^{*}\right)$ of the monad $p_{0 *} q_{0}^{*}$ in $D_{\text {perf }}^{G_{\overline{0}}, \geq}\left(\mathfrak{G}_{1,1}^{\bullet}\right)$ to $D_{\mathbf{G}_{\mathbf{O}}}\left(\mathbf{G r} \times\left(\mathbf{V}_{0} \backslash t \mathbf{V}_{0}\right)\right)$. For the modules $V_{1, \boldsymbol{\lambda}} \otimes \mathfrak{G}_{1,1}^{\bullet} \otimes V_{2, \boldsymbol{\mu}}, V_{1, \boldsymbol{\lambda}^{\prime}} \otimes \mathfrak{G}_{1,1}^{\bullet} \otimes V_{2, \boldsymbol{\mu}^{\prime}}$ over the monad $p_{0 *} q_{0}^{*}$ (here both $\boldsymbol{\lambda}, \boldsymbol{\lambda}^{\prime}$ are partitions), we have

$\operatorname{Hom}_{D\left(p_{0 *} q_{0}^{*}\right)}\left(V_{1, \boldsymbol{\lambda}} \otimes \mathfrak{G}_{1,1}^{\bullet} \otimes V_{2, \boldsymbol{\mu}}, V_{1, \boldsymbol{\lambda}^{\prime}} \otimes \mathfrak{G}_{1,1}^{\bullet} \otimes V_{2, \boldsymbol{\mu}^{\prime}}\right)=\left(V_{1, \boldsymbol{\lambda}}^{*} \otimes V_{1, \boldsymbol{\lambda}^{\prime}}^{\prime} \otimes \mathfrak{F}^{\bullet} \otimes\left(V_{2, \boldsymbol{\mu}}^{*} \otimes V_{2, \boldsymbol{\mu}^{\prime}}\right)\right)^{G_{Q}}$.

Recall the equivalence $D_{\mathbf{G}_{\mathbf{O}}}\left(\mathbf{G r} \times\left(\mathbf{V}_{0} \backslash t \mathbf{V}_{0}\right)\right) \cong D_{\mathbf{M}_{\mathbf{O}}}(\mathbf{G r})$. By an abuse of notation, we will denote the composed equivalence $D\left(p_{0 *} q_{0}^{*}\right) \stackrel{\sim}{\longrightarrow} D_{\mathbf{M}_{\mathbf{O}}}(\mathbf{G r})$ also by $\Phi^{\prime}$.

On the other hand, we consider a full subcategory $D_{\text {perf }}^{\bar{G}_{\overline{0}}, \geq 0}\left(\overline{\mathfrak{G}}^{\bullet}\right) \subset D_{\text {perf }}^{\bar{G}_{\overline{0}}}\left(\overline{\mathfrak{G}}^{\bullet}\right)$ generated by the free modules $\bar{V}_{1, \boldsymbol{\lambda}} \otimes \overline{\mathfrak{G}} \bullet \otimes V_{2, \boldsymbol{\mu}}$ for partitions $\boldsymbol{\lambda}$ (of length $\leq N-1$ ). We construct an equivalence $\Phi: D\left(p_{0 *} q_{0}^{*}\right) \stackrel{\sim}{\longrightarrow} D_{\text {perf }}^{\bar{G}_{\overline{0}}, \geq 0}\left(\overline{\mathfrak{G}^{\bullet}}\right)$ as follows. We consider the variety $\mathcal{P}$ of sixtuples

$$
\begin{aligned}
& A \in \operatorname{Hom}\left(V_{1}, V_{2}\right), \quad B \in \operatorname{Hom}\left(V_{2}, V_{1}\right), \bar{A} \in \operatorname{Hom}\left(\bar{V}_{1}, V_{2}\right), \\
& \bar{B} \in \operatorname{Hom}\left(V_{2}, \bar{V}_{1}\right), \quad b \in \operatorname{Hom}\left(V_{1}, \bar{V}_{1}\right), a \in \operatorname{Hom}\left(\bar{V}_{1}, V_{1}\right),
\end{aligned}
$$

such that $A=\bar{A} b, \bar{B}=b B, a=B \bar{A}$. Clearly,

$$
\mathcal{P} \simeq \operatorname{Hom}\left(V_{1}, \bar{V}_{1}\right) \times \operatorname{Hom}\left(\bar{V}_{1}, V_{2}\right) \times \operatorname{Hom}\left(V_{2}, V_{1}\right)
$$

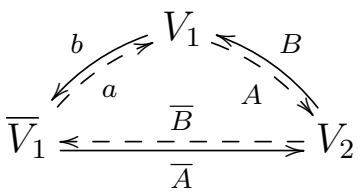

We have the natural morphisms

$\mathrm{p}: \mathcal{P} \rightarrow \operatorname{Hom}\left(V_{1}, V_{2}\right) \times \operatorname{Hom}\left(V_{2}, V_{1}\right)=\Pi \mathfrak{g}_{1}^{*}, \mathrm{q}: \mathcal{P} \rightarrow \operatorname{Hom}\left(\bar{V}_{1}, V_{2}\right) \times \operatorname{Hom}\left(V_{2}, \bar{V}_{1}\right)=\Pi \overline{\mathfrak{g}}_{1}^{*} ;$

$$
\mathrm{p}(A, \bar{A}, a, b, B, \bar{B})=(A, B), \mathrm{q}(A, \bar{A}, a, b, B, \bar{B})=(\bar{A}, \bar{B}) .
$$

The variety $\mathcal{P}$ is acted upon by $G_{\mathcal{P}}:=\operatorname{GL}\left(V_{1}\right) \times \operatorname{GL}\left(\bar{V}_{1}\right) \times \operatorname{GL}\left(V_{2}\right)$ :

$$
\left(g_{1}, \bar{g}_{1}, g_{2}\right)(A, \bar{A}, a, b, B, \bar{B})=\left(g_{2} A g_{1}^{-1}, g_{2} \bar{A} \bar{g}_{1}^{-1}, g_{1} a \bar{g}_{1}^{-1}, \bar{g}_{1} b g_{1}^{-1}, g_{1} B g_{2}^{-1}, \bar{g}_{1} \bar{B} g_{2}^{-1}\right) \text {. }
$$

We have the natural morphisms

$$
\mathrm{p}: G_{\mathcal{P}} \rightarrow G_{\overline{0}}, \mathrm{p}\left(g_{1}, \bar{g}_{1}, g_{2}\right)=\left(g_{1}, g_{2}\right) ; \mathrm{q}: G_{\mathcal{P}} \rightarrow \bar{G}_{\overline{0}}, \mathbf{q}\left(g_{1}, \bar{g}_{1}, g_{2}\right)=\left(\bar{g}_{1}, g_{2}\right) \text {. }
$$

Clearly, the morphisms p: $\mathcal{P} \rightarrow \Pi \mathfrak{g}_{1}^{*}$, q: $\mathcal{P} \rightarrow \Pi \overline{\mathfrak{g}}_{\overline{1}}^{*}$ are equivariant with respect to $\mathrm{p}: G_{\mathcal{P}} \rightarrow G_{\overline{0}}, \mathrm{q}: G_{\mathcal{P}} \rightarrow \bar{G}_{\overline{0}}$. Hence we have the convolution functor $\mathrm{q}_{*} \mathrm{p}^{*}: \operatorname{Coh}^{G_{\overline{0}}}\left(\Pi \mathfrak{g}_{\overline{1}}^{*}\right)=\operatorname{Coh}\left(G_{\overline{0}} \backslash \Pi \mathfrak{g}_{1}^{*}\right) \stackrel{\mathrm{p}^{*}}{\rightarrow} \operatorname{Coh}\left(G_{\mathcal{P}} \backslash \mathcal{P}\right) \stackrel{\mathrm{q}_{*}}{\rightarrow} \operatorname{Coh}\left(\bar{G}_{\overline{0}} \backslash \Pi \overline{\mathfrak{g}}_{\overline{1}}^{*}\right)=\operatorname{Coh}^{\bar{G}_{\overline{0}}}\left(\Pi \overline{\mathfrak{g}}_{\overline{1}}^{*}\right)$ 
(in particular, $\mathbf{q}_{*}$ involves taking $\mathrm{GL}\left(V_{1}\right)$-invariants). We will actually need the same named functor $\mathrm{q}_{*} \mathrm{p}^{*}: D_{\text {perf }}^{G_{\overline{0}}}\left(\mathfrak{G}_{1,1}^{\bullet}\right) \rightarrow D_{\text {perf }}^{\bar{G}_{\overline{0}}}\left(\overline{\mathfrak{G}}^{\bullet}\right)$ defined similarly using the dg-algebra with trivial differential

$$
\operatorname{Sym}\left(\operatorname{Hom}\left(V_{1}, V_{2}\right)[-1] \oplus \operatorname{Hom}\left(\bar{V}_{1}, V_{1}\right)[-1]\right) \otimes \operatorname{Sym} \operatorname{Hom}\left(\bar{V}_{1}, V_{1}\right)
$$

(the grading on $\operatorname{Sym} \operatorname{Hom}\left(\bar{V}_{1}, V_{1}\right)$ is trivial, and if we disregard the grading, then $\left.\operatorname{Sym}\left(\operatorname{Hom}\left(V_{1}, V_{2}\right)[-1] \oplus \operatorname{Hom}\left(\bar{V}_{1}, V_{1}\right)[-1]\right) \otimes \operatorname{Sym} \operatorname{Hom}\left(\bar{V}_{1}, V_{1}\right) \simeq \mathbb{C}[\mathcal{P}]\right)$.

Now recall that $D\left(p_{0 *} q_{0}^{*}\right)$ is a full subcategory of $D_{\text {perf }}^{G_{\overline{0}}}\left(\mathfrak{G}_{1,1}^{\bullet}\right)$. The desired functor $\Phi: D\left(p_{0 *} q_{0}^{*}\right) \rightarrow D_{\text {perf }}^{\bar{G}_{\overline{0}}}\left(\overline{\mathfrak{G}^{\bullet}}\right)$ is nothing but the restriction of $\mathbf{q}_{*} \mathbf{p}^{*}$ to the full subcategory $D\left(p_{0 *} q_{0}^{*}\right) \subset D_{\text {perf }}^{G_{\overline{0}}}\left(\mathfrak{G}_{1,1}^{\bullet}\right)$. The full subcategory $D\left(p_{0 *} q_{0}^{*}\right)$ is generated by the objects $p_{0 *} q_{0}^{*}\left(V_{1, \boldsymbol{\lambda}} \otimes \mathfrak{G}_{1,1}^{\bullet} \otimes V_{2, \boldsymbol{\mu}}\right) \cong_{D\left(p_{0 *} q_{0}^{*}\right)} V_{1, \boldsymbol{\lambda}} \otimes \mathfrak{G}_{1,1}^{\bullet} \otimes V_{2, \boldsymbol{\mu}}$ (the LHS and the RHS are isomorphic in the Kleisli category $\left.D\left(p_{0 *} q_{0}^{*}\right)\right)$ with $\boldsymbol{\lambda}$ running through the set of partitions. For a partition $\boldsymbol{\lambda}$ we have

$$
\Phi\left(V_{1, \boldsymbol{\lambda}} \otimes \mathfrak{G}_{1,1}^{\bullet} \otimes V_{2, \boldsymbol{\mu}}\right)=\bar{V}_{1, \boldsymbol{\lambda}} \otimes \overline{\mathfrak{G}}^{\bullet} \otimes V_{2, \boldsymbol{\mu}}
$$

in notation of Section 3.13 (i.e. if $\boldsymbol{\lambda}=\left(\lambda_{1} \geq \lambda_{2} \geq \ldots \geq \lambda_{N} \geq 0\right)$ and $\lambda_{N}>0$, then $\Phi\left(V_{1, \boldsymbol{\lambda}} \otimes \mathfrak{G}_{1,1}^{\bullet} \otimes V_{2, \boldsymbol{\mu}}\right)=0$, and if $\lambda_{N}=0$, then

$$
\left.\Phi\left(V_{1, \boldsymbol{\lambda}} \otimes \mathfrak{G}_{1,1}^{\bullet} \otimes V_{2, \boldsymbol{\mu}}\right)=\bar{V}_{1,\left(\lambda_{1} \geq \ldots \geq \lambda_{N-1}\right)} \otimes \overline{\mathfrak{G}} \bullet V_{2, \boldsymbol{\mu}} .\right)
$$

Hence $\Phi\left(V_{1, \boldsymbol{\lambda}} \otimes \mathfrak{G}_{1,1}^{\bullet} \otimes V_{2, \boldsymbol{\mu}}\right)$ actually lies in $D_{\text {perf }}^{\bar{G}_{\overline{0}}, \geq 0}\left(\overline{\mathfrak{G}^{\bullet}}\right) \subset D_{\text {perf }}^{\bar{G}_{\overline{0}}}\left(\overline{\mathfrak{G}^{\bullet}}\right)$. It remains to check that $\Phi$ is fully faithful.

For partitions $\boldsymbol{\lambda}, \boldsymbol{\lambda}^{\prime}$, we have to check that the following morphism is an isomorphism:

$$
\begin{aligned}
& \left(V_{1, \boldsymbol{\lambda}}^{*} \otimes p_{0 *} q_{0}^{*}\left(V_{1, \boldsymbol{\lambda}^{\prime}}^{\prime} \otimes \mathbb{C}\left[\operatorname{Hom}\left(V_{1}^{\prime}, V_{2}\right) \times \operatorname{Hom}\left(V_{2}, V_{1}^{\prime}\right)\right] \otimes V_{2, \boldsymbol{\mu}^{\prime}}\right) \otimes V_{2, \boldsymbol{\mu}}^{*}\right)^{\mathrm{GL}\left(V_{1}\right) \times \mathrm{GL}\left(V_{2}\right)} \\
& :=\left(V_{1, \boldsymbol{\lambda}}^{*} \otimes V_{1, \boldsymbol{\lambda}^{\prime}}^{\prime} \otimes \mathbb{C}\left[Q_{0}\right] \otimes\left(V_{2, \boldsymbol{\mu}}^{*} \otimes V_{2, \boldsymbol{\mu}^{\prime}}\right)\right)^{G_{Q}}=\left(\mathbb{S}_{\boldsymbol{\lambda}} V_{1}^{*} \otimes \mathbb{S}_{\boldsymbol{\lambda}^{\prime}} V_{1}^{\prime} \otimes \mathbb{C}\left[Q_{0}\right] \otimes\left(V_{2, \boldsymbol{\mu}}^{*} \otimes V_{2, \boldsymbol{\mu}^{\prime}}\right)\right)^{G_{Q}} \\
& \stackrel{\sim}{\longrightarrow}\left(\mathbb{S}_{\boldsymbol{\lambda}} \bar{V}_{1}^{*} \otimes \mathbb{S}_{\boldsymbol{\lambda}^{\prime}} \bar{V}_{1} \otimes \mathbb{C}\left[\operatorname{Hom}\left(\bar{V}_{1}, V_{2}\right) \times \operatorname{Hom}\left(V_{2}, \bar{V}_{1}\right)\right] \otimes\left(V_{2, \boldsymbol{\mu}}^{*} \otimes V_{2, \boldsymbol{\mu}^{\prime}}\right)\right)^{\mathrm{GL}\left(\bar{V}_{1}\right) \times \mathrm{GL}\left(V_{2}\right)} .
\end{aligned}
$$

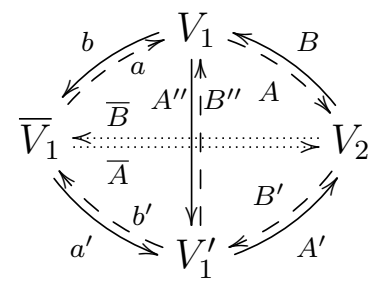

The desired isomorphism is equal to the composition of the following three. The first one is induced by

$$
\mathbb{C}\left[\operatorname{Hom}_{\leq N-1}\left(V_{1}, V_{1}^{\prime}\right)\right] \stackrel{\sim}{\longrightarrow}\left(\mathbb{C}\left[\operatorname{Hom}\left(V_{1}, \bar{V}_{1}\right)\right] \otimes \mathbb{C}\left[\operatorname{Hom}\left(\bar{V}_{1}, V_{1}^{\prime}\right)\right]\right)^{\mathrm{GL}\left(\bar{V}_{1}\right)},
$$


see Lemma 3.13.1(a). The second one is induced by the inverse of

$$
\mathbb{S}_{\boldsymbol{\lambda}^{\prime}} \bar{V}_{1} \otimes \mathbb{C}\left[\operatorname{Hom}\left(\bar{V}_{1}, V_{2}\right)\right] \stackrel{\sim}{\longrightarrow}\left(\mathbb{C}\left[\operatorname{Hom}\left(\bar{V}_{1}, V_{1}^{\prime}\right) \otimes \mathbb{S}_{\boldsymbol{\lambda}^{\prime}} V_{1}^{\prime} \otimes \mathbb{C}\left[\operatorname{Hom}\left(V_{1}^{\prime}, V_{2}\right)\right]\right)^{\mathrm{GL}\left(V_{1}^{\prime}\right)},\right.
$$

see Lemma 3.13.1(b). The third one is induced by the inverse of

$$
\mathbb{C}\left[\operatorname{Hom}\left(V_{2}, \bar{V}_{1}\right)\right] \otimes \mathbb{S}_{\boldsymbol{\lambda}^{*}} \bar{V}_{1} \stackrel{\sim}{\longrightarrow}\left(\mathbb{C}\left[\operatorname{Hom}\left(V_{2}, V_{1}\right) \otimes \mathbb{S}_{\boldsymbol{\lambda}^{*}} V_{1} \otimes \mathbb{C}\left[\operatorname{Hom}\left(V_{1}, \bar{V}_{1}\right)\right]\right)^{\mathrm{GL}\left(V_{1}\right)},\right.
$$

see Lemma 3.13.1(c).

4.4. Proof of Theorem 4.1.1. We consider an F-linear automorphism $\xi$ of $\mathbf{V}: \xi\left(e_{1}\right)=e_{1}, \xi\left(e_{i}\right)=t e_{i}$ for $i=2, \ldots, N$, and the same named induced automorphism of $\mathbf{G r}$. It is given by the action of an element $\operatorname{diag}(1, t, \ldots, t)$ of the diagonal Cartan torus $\mathbf{T}_{\mathbf{F}}$. Note that $\operatorname{diag}(1, t, \ldots, t) \mathbf{M}_{\mathbf{O}} \operatorname{diag}\left(1, t^{-1}, \ldots, t^{-1}\right) \supset \mathbf{M}_{\mathbf{O}}$. Hence $\xi_{*}$ acts on the equivariant category $D_{\mathbf{M}_{\mathbf{O}}}(\mathbf{G r})$ (by restricting equivariance from $\operatorname{diag}(1, t, \ldots, t) \mathbf{M}_{\mathbf{O}} \operatorname{diag}\left(1, t^{-1}, \ldots, t^{-1}\right)$ to $\left.\mathbf{M}_{\mathbf{O}}\right)$. Clearly, $\xi_{*}$ acts on the equivariant category $D_{\mathrm{GL}(N-1, \mathbf{O})}(\mathbf{G r})$ as well. Recall that $\operatorname{Res}_{\mathbf{M}_{\mathbf{O}}}^{\mathrm{GL}(N-1, \mathbf{O})}: D_{\mathbf{M}_{\mathbf{O}}}(\mathbf{G r}) \rightarrow D_{\mathrm{GL}(N-1, \mathbf{O})}(\mathbf{G r})$ denotes the functor of restriction of equivariance under $\mathrm{GL}(N-1, \mathbf{O}) \hookrightarrow \mathbf{M}_{\mathbf{O}}$. We denote by $\mathrm{Av}_{\mathrm{GL}(N-1, \mathbf{O})}^{\mathbf{M}_{\mathbf{O}}}: D_{\mathrm{GL}(N-1, \mathbf{O})}(\mathbf{G r}) \rightarrow D_{\mathbf{M}_{\mathbf{O}}}(\mathbf{G r})$ the corresponding right adjoint *-averaging functor.

Lemma 4.4.1. (a) Given $\mathcal{F} \in D_{\mathrm{GL}(N-1, \mathbf{O})}(\mathbf{G r})$, for $n \gg 0$ the canonical mor-

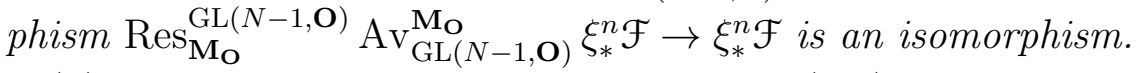

(b) $\xi_{*}$ is an auto-equivalence of $D_{\mathrm{GL}(N-1, \mathbf{O})}(\mathbf{G r})$, and hence the natural functor from $D_{\mathrm{GL}(N-1, \mathbf{O})}(\mathbf{G r})$ to colim $_{\xi_{*}} D_{\mathrm{GL}(N-1, \mathbf{O})}(\mathbf{G r})$ is an equivalence.

(c) The restriction of equivariance functor $\operatorname{Res}_{\mathbf{M}_{\mathbf{O}}}^{\mathrm{GL}(N-1, \mathbf{O})}: D_{\mathbf{M}_{\mathbf{O}}}(\mathbf{G r}) \rightarrow$ $D_{\mathrm{GL}(N-1, \mathbf{O})}(\mathbf{G r})$ induces the same named equivalence of the colimits $\operatorname{Res}_{\mathbf{M}_{\mathbf{O}}}^{\mathrm{GL}(N-1, \mathbf{O})}: \operatorname{colim}_{\xi_{*}} D_{\mathbf{M}_{\mathbf{O}}}(\mathbf{G r}) \rightarrow \operatorname{colim}_{\xi_{*}} D_{\mathrm{GL}(N-1, \mathbf{O})}(\mathbf{G r}) \cong D_{\mathrm{GL}(N-1, \mathbf{O})}(\mathbf{G r})$.

Proof. It suffices to prove (a) for an irreducible perverse $\mathcal{F} \in D_{\mathrm{GL}(N-1, \mathbf{O})}(\mathbf{G r})$. This in turn follows from the fact that for any $\operatorname{GL}(N-1, \mathbf{O})$-orbit

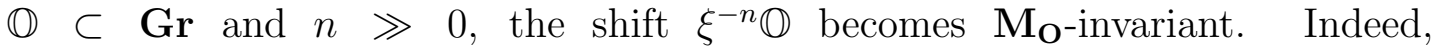
$\mathbf{M}_{\mathbf{O}}$ is generated by its radical $\mathbf{U}_{\mathbf{O}}$ and $\mathrm{GL}(N-1, \mathbf{O})$. As $n$ grows, $\mathbf{U}_{\mathbf{O}}^{(n)}:=\operatorname{diag}\left(1, t^{-n}, \ldots, t^{-n}\right) \mathbf{U}_{\mathbf{O}} \operatorname{diag}\left(1, t^{n}, \ldots, t^{n}\right)$ forms a system of shrinking subgroups of $\mathbf{U}_{\mathbf{O}}$, and we take $n$ big enough so that the action of $\mathbf{U}_{\mathbf{O}}^{(n)}$ on $\mathbb{O}$ is trivial (recall that the action of $\mathbf{U}_{\mathbf{O}}$ on any Schubert subvariety of $\mathbf{G r}$ factors through a finite dimensional quotient group).

(b) is evident, and (c) follows from (a) and (b).

On the coherent side we consider an endofunctor $\eta$ of $D_{\text {perf }}^{\bar{G}_{\overline{0}}, \geq 0}\left(\overline{\mathfrak{G}}^{\bullet}\right)$ obtained by tensoring with the polynomial representation $\operatorname{det}\left(\bar{V}_{1}\right)$ of $\operatorname{GL}\left(\bar{V}_{1}\right)$. The full 
embedding $D_{\text {perf }}^{\bar{G}_{\overline{0}}, \geq 0}\left(\overline{\mathfrak{G}}^{\bullet}\right) \hookrightarrow D_{\text {perf }}^{\bar{G}_{\overline{0}}}\left(\overline{\mathfrak{G}}^{\bullet}\right)$ gives rise to an equivalence of colimits $\operatorname{colim}_{\eta} D_{\text {perf }}^{\bar{G}_{\overline{0}}, \geq 0}\left(\overline{\mathfrak{G}^{\bullet}}\right) \stackrel{\sim}{\longrightarrow} \operatorname{colim}_{\eta} D_{\text {perf }}^{\bar{G}_{\overline{0}}}\left(\overline{\mathfrak{G}^{\bullet}}\right) \cong D_{\text {perf }}^{\bar{G}_{\overline{0}}}\left(\overline{\mathfrak{G}}^{\bullet}\right)$.

Composing the inverse of $\Phi$ with $\Phi^{\prime}$ (notation of Section 4.3) we obtain an equivalence $\bar{\Phi}^{\prime}: D_{\text {perf }}^{\bar{G}_{\overline{0}}, \geq 0}\left(\overline{\mathfrak{G}^{\bullet}}\right) \stackrel{\sim}{\longrightarrow} D_{\mathbf{M}_{\mathbf{O}}}(\mathbf{G r})$. According to Lemma 4.2 .2 , the equivalence $\bar{\Phi}^{\prime}: D_{\text {perf }}^{\bar{G}_{\overline{0}}, \geq 0}\left(\overline{\mathfrak{G}^{\bullet}}\right) \stackrel{\sim}{\longrightarrow} D_{\mathbf{M}_{\mathbf{O}}}(\mathbf{G r})$ intertwines the endofunctors $\eta$ and $\xi_{*}$, and hence induces the desired equivalence $\bar{\Phi}: D_{\text {perf }}^{\bar{G}_{\overline{0}}}\left(\overline{\mathfrak{G}}^{\bullet}\right) \stackrel{\sim}{\longrightarrow} D_{\mathrm{GL}(N-1, \mathbf{O})}(\mathbf{G r})$ between the colimits. Theorem 4.1.1 is proved.

\section{LOOP ROTATION AND QUANTIZATION}

5.1. Graded differential operators and convolutions. We have $H_{\mathbb{G}_{m}}^{\bullet}(\mathrm{pt})=\mathbb{C}[\hbar]$. We consider the algebra $\mathfrak{D}$ of "graded differential operators" on $\operatorname{Hom}\left(V_{2}, V_{1}\right)$ : a $\mathbb{C}[\hbar]$-algebra generated by $\operatorname{Hom}\left(V_{2}, V_{1}\right)$ and $\operatorname{Hom}\left(V_{1}, V_{2}\right)$ with relations $\left[h, h^{\prime}\right]=\left[f, f^{\prime}\right]=0,[h, f]=\langle h, f\rangle \hbar$ for $h, h^{\prime} \in \operatorname{Hom}\left(V_{2}, V_{1}\right), f, f^{\prime} \in \operatorname{Hom}\left(V_{1}, V_{2}\right)$. It is equipped with the grading $\operatorname{deg} f=\operatorname{deg} h=1, \operatorname{deg} \hbar=2$. We denote by $\mathfrak{D}_{1,1}^{\bullet}$ this graded algebra viewed as a dg-algebra with trivial differential. We will also need two more versions of $\mathfrak{D}_{1,1}^{\bullet}$ differing by the gradings of generators: in $\mathfrak{D}_{0,2}^{\bullet}$ we set $\operatorname{deg} f=0, \operatorname{deg} h=2$, while in $\mathfrak{D}_{2,0}^{\bullet}$ we set $\operatorname{deg} f=2, \operatorname{deg} h=0$.

Recall the setup and notation of Section 3.4. So we have another copy $V_{1}^{\prime}$ of $V_{1}$, we rebaptize the algebra $\mathfrak{D}$ of "graded differential operators" on $\operatorname{Hom}\left(V_{2}, V_{1}\right)$ as $\mathfrak{D}_{21}$, and along with it we consider $\mathfrak{D}_{21^{\prime}}$ ("graded differential operators" on $\operatorname{Hom}\left(V_{2}, V_{1}^{\prime}\right)$ ) and $\mathfrak{D}_{1^{\prime} 1}$ ("graded differential operators" on $\operatorname{Hom}\left(V_{1}^{\prime}, V_{1}\right)$ ). Note that $\mathfrak{D}_{21} \cong \mathfrak{D}_{12}$ ("graded differential operators" on $\operatorname{Hom}\left(V_{1}, V_{2}\right)$ ), and similarly $\mathfrak{D}_{21^{\prime}} \cong \mathfrak{D}_{1^{\prime} 2}$, and $\mathfrak{D}_{1^{\prime} 1} \cong \mathfrak{D}_{11^{\prime}}$.

We have morphisms

$$
\begin{aligned}
& \mathrm{m}^{A}: \operatorname{Hom}\left(V_{1}^{\prime}, V_{2}\right) \times \operatorname{Hom}\left(V_{1}, V_{1}^{\prime}\right) \rightarrow \operatorname{Hom}\left(V_{1}, V_{2}\right),\left(A^{\prime}, A^{\prime \prime}\right) \mapsto A^{\prime} A^{\prime \prime}, \\
& \mathrm{m}^{B}: \operatorname{Hom}\left(V_{1}^{\prime}, V_{1}\right) \times \operatorname{Hom}\left(V_{2}, V_{1}^{\prime}\right) \rightarrow \operatorname{Hom}\left(V_{2}, V_{1}\right),\left(B^{\prime \prime}, B^{\prime}\right) \mapsto B^{\prime \prime} B^{\prime} .
\end{aligned}
$$

They give rise to the functors

$$
\begin{aligned}
& D_{\text {perf }}^{\mathrm{GL}\left(V_{1}^{\prime}\right) \times \mathrm{GL}\left(V_{2}\right)}\left(\mathfrak{D}_{1^{\prime} 2}\right) \times D_{\text {perf }}^{\mathrm{GL}\left(V_{1}\right) \times \mathrm{GL}\left(V_{1}^{\prime}\right)}\left(\mathfrak{D}_{11^{\prime}}\right) \rightarrow D_{\text {perf }}^{\mathrm{GL}\left(V_{1}\right) \times \mathrm{GL}\left(V_{2}\right)}\left(\mathfrak{D}_{12}\right), \\
&\left(\mathcal{M}_{1^{\prime} 2}, \mathcal{M}_{11^{\prime}}\right) \mapsto\left(\mathrm{m}_{*}^{A}\left(\mathcal{M}_{1^{\prime} 2} \otimes \mathcal{M}_{11^{\prime}}\right)\right)^{\mathrm{GL}\left(V_{1}^{\prime}\right)}, \\
& D_{\text {perf }}^{\mathrm{GL}\left(V_{1}^{\prime}\right) \times \mathrm{GL}\left(V_{1}\right)}\left(\mathfrak{D}_{1^{\prime} 1}\right) \times D_{\text {perf }}^{\mathrm{GL}\left(V_{2}\right) \times \mathrm{GL}\left(V_{1}^{\prime}\right)}\left(\mathfrak{D}_{21^{\prime}}\right) \rightarrow D_{\text {perf }}^{\mathrm{GL}\left(V_{2}\right) \times \mathrm{GL}\left(V_{1}\right)}\left(\mathfrak{D}_{21}\right), \\
&\left(\mathcal{M}_{1^{\prime} 1}, \mathcal{M}_{21^{\prime}}\right) \mapsto\left(\mathrm{m}_{*}^{B}\left(\mathcal{M}_{1^{\prime} 1} \otimes \mathcal{M}_{21^{\prime}}\right)\right)^{\mathrm{GL}\left(V_{1}^{\prime}\right)}
\end{aligned}
$$

(here $\mathrm{m}_{*}^{A}, \mathrm{~m}_{*}^{B}$ stand for the direct images in the category of $\mathfrak{D}$-modules). 
We will actually need the corresponding functors on the categories of $\mathrm{dg}$ modules

$\stackrel{A}{*}: D_{\text {perf }}^{G_{\overline{0}}}\left(\mathfrak{D}_{2,0}^{\bullet}\right) \times D_{\text {perf }}^{G_{\overline{0}}}\left(\mathfrak{D}_{2,0}^{\bullet}\right) \rightarrow D_{\text {perf }}^{G_{\overline{0}}}\left(\mathfrak{D}_{2,0}^{\bullet}\right), \stackrel{B}{*}: D_{\text {perf }}^{G_{\overline{0}}}\left(\mathfrak{D}_{0,2}^{\bullet}\right) \times D_{\text {perf }}^{G_{\overline{0}}}\left(\mathfrak{D}_{0,2}^{\bullet}\right) \rightarrow D_{\text {perf }}^{G_{\overline{0}}}\left(\mathfrak{D}_{0,2}^{\bullet}\right)$.

The multiplicative group $\mathbb{G}_{m}$ acts on $\mathbf{G r} \times \mathbf{V}$ as loop rotations. The goal of this section is the following

Theorem 5.1.1. There exist monoidal equivalences of triangulated categories

$$
\begin{aligned}
& \left(D_{\text {perf }}^{G_{\overline{0}}}\left(\mathfrak{D}_{2,0}^{\bullet}\right), \stackrel{A}{*}\right) \underset{\Phi_{\hbar}^{2,0}}{\stackrel{\sim}{\longrightarrow}}\left(D_{! \mathbf{G}_{\mathbf{O}} \rtimes \mathbb{C} \times}(\mathbf{G r} \times \mathbf{V}), \stackrel{!}{\circledast}\right) \\
& \text { 2Џ } \varrho_{\text {right }} \quad 2 \downarrow \varrho_{\text {right }} \\
& D_{\text {perf }}^{G_{\overline{0}}}\left(\mathfrak{D}_{1,1}^{\bullet}\right) \quad \underset{\Phi_{\hbar}^{1,1}}{\stackrel{\sim}{\longrightarrow}} \quad D_{! * \mathbf{G}_{\mathbf{O}} \rtimes \mathbb{C}^{\times}}(\mathbf{G r} \times \mathbf{V})
\end{aligned}
$$

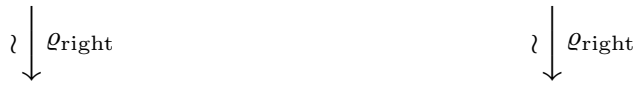

$$
\begin{aligned}
& \left(D_{\text {perf }}^{G_{\overline{0}}}\left(\mathfrak{D}_{0,2}^{\bullet}\right), \quad \stackrel{B}{*}\right) \underset{\Phi_{\hbar}^{0,2}}{\stackrel{\sim}{\longrightarrow}}\left(D_{* \mathbf{G}_{\mathbf{O}} \rtimes \mathbb{C} \times}(\mathbf{G r} \times \mathbf{V}), \quad * *\right.
\end{aligned}
$$

(the vertical and the middle row equivalences are not monoidal). The horizontal equivalences commute with the actions of the monoidal spherical Hecke category $\operatorname{Perv}_{\mathbf{G}_{\mathbf{O}} \rtimes \mathbb{C} \times}(\mathbf{G r}) \cong \operatorname{Rep}\left(\mathrm{GL}_{N}\right)$ by the left and right convolutions.

The proof will be given in Section 5.3.

5.2. Construction of equivalences. We set $\mathfrak{E}_{\hbar}^{\bullet}:=\operatorname{Ext}_{D_{I_{* \mathbb{G}_{\mathrm{O}} \rtimes \mathbb{G}_{m}}}^{\bullet}(\mathbf{G r} \times \mathbf{V})}\left(E_{0}, E_{0}\right)$ (a dg-algebra with trivial differential). Since it is an Ext-algebra in the deequivariantized category, it is automatically equipped with an action of $\mathrm{GL}_{N} \times$ $\mathrm{GL}_{N}=\mathrm{GL}\left(V_{1}\right) \times \mathrm{GL}\left(V_{2}\right)=G_{\overline{0}}$, and we can consider the corresponding triangulated category $D_{\text {perf }}^{G_{\overline{0}}}\left(\mathfrak{E}_{\hbar}^{\bullet}\right)$. Similarly to Lemma 3.9.2, there is a canonical equivalence $D_{\text {perf }}^{G_{\overline{0}}}\left(\mathfrak{E}_{\hbar}^{\bullet} \stackrel{\sim}{\longrightarrow} D_{! * \mathbf{G}_{\mathbf{O}} \rtimes \mathbb{G}_{m}}(\mathbf{G r} \times \mathbf{V})\right.$. It remains to construct an isomorphism $\phi_{\hbar}^{\bullet}: \mathfrak{D}_{1,1}^{\bullet} \stackrel{\sim}{\longrightarrow} \mathfrak{E}_{\hbar}^{\bullet}$.

Note that $\mathfrak{E}_{\hbar}^{\bullet}$ is a $\mathbb{C}[\hbar]$-algebra, and $\mathfrak{E}_{\hbar}^{\bullet} /(\hbar=0)=\mathfrak{E}^{\bullet}=\mathfrak{G}_{1,1}^{\bullet}=\operatorname{Sym}\left(\mathfrak{g}_{1}[-1]\right)$, so that $\mathfrak{E} \bullet$ acquires a Poisson bracket from this deformation. We claim that this Poisson bracket arises from the canonical symplectic form on $\mathfrak{g}_{1}^{*}$. Indeed, the isomorphism $\phi^{*}$ (see the proof of Lemma 3.11.1) over $\operatorname{Isom}\left(V_{2}, V_{1}\right) \times \operatorname{Hom}\left(V_{1}, V_{2}\right)$ and $\operatorname{Hom}\left(V_{2}, V_{1}\right) \times \operatorname{Isom}\left(V_{1}, V_{2}\right)$ is Poisson. Here the Poisson structure on these open subsets arises from the deformations $\mathfrak{A}_{\hbar}^{\bullet}, \mathfrak{B}_{\hbar}^{\bullet}$ which in turn arise from the loop-rotation-equivariant Satake category $D_{\mathbf{G}_{\mathbf{O} \rtimes \mathbb{G}_{m}}}(\mathbf{G r})$. The corresponding Poisson brackets are the standard ones on the cotangent bundles $T^{*} \operatorname{Isom}\left(V_{2}, V_{1}\right), T^{*} \operatorname{Isom}\left(V_{1}, V_{2}\right)$ as follows from [BF, Theorem 5]. 
Now $\mathfrak{D}_{1,1}^{\bullet}$ is a unique graded $\mathbb{C}[\hbar]$-algebra with $\mathfrak{D}_{1,1}^{\bullet} /(\hbar=0)=\operatorname{Sym}\left(\mathfrak{g}_{\overline{1}}[-1]\right)$ such that the corresponding Poisson bracket on $\operatorname{Sym}\left(\mathfrak{g}_{1}[-1]\right)$ is the standard one. Thus $\phi_{\hbar}^{\bullet}$ and $\Phi_{\hbar}^{1,1}$ are constructed.

5.3. Restriction to $\mathbf{G r} \times \mathbf{V}_{0}$ with loop rotation. Similarly to Section 3.12, our goal in this section is a description in terms of equivalence of Theorem 5.1.1 of the endofunctors $\bar{\jmath}_{0 *} \bar{J}_{0}^{!}, \quad \jmath_{0 *} \jmath_{0}^{!}: D_{! * \mathbf{G}_{\mathbf{O}} \rtimes \mathbb{C}^{\times}}(\mathbf{G r} \times \mathbf{V}) \rightarrow D_{! * \mathbf{G}_{\mathbf{O}} \rtimes \mathbb{C}^{\times}}(\mathbf{G r} \times \mathbf{V})$. To this end we will need the algebra $\mathfrak{H}$ of "graded differential operators" on $\operatorname{Hom}\left(V_{1}, V_{2}\right) \oplus \operatorname{Hom}\left(V_{1}, V_{1}^{\prime}\right) \oplus \operatorname{Hom}\left(V_{1}^{\prime}, V_{2}\right)$ defined similarly to $\mathfrak{D}$ of Section 5.1. It is equipped with the grading where the generators from $\operatorname{Hom}\left(V_{2}, V_{1}\right), \operatorname{Hom}\left(V_{1}, V_{2}\right), \operatorname{Hom}\left(V_{1}^{\prime}, V_{2}\right), \operatorname{Hom}\left(V_{2}, V_{1}^{\prime}\right)$ have degree 1 , while the generators from $\operatorname{Hom}\left(V_{1}, V_{1}^{\prime}\right), \operatorname{Hom}\left(V_{1}^{\prime}, V_{1}\right)$ have degrees 2,0 respectively, and $\operatorname{deg} \hbar=2$. We denote by $\mathfrak{H}^{\bullet}$ this graded algebra viewed as a dg-algebra with trivial differential. If we denote the similar dg-algebras of "graded differential operators" on $\operatorname{Hom}\left(V_{1}, V_{2}\right), \operatorname{Hom}\left(V_{1}, V_{1}^{\prime}\right), \operatorname{Hom}\left(V_{1}^{\prime}, V_{2}\right)$ by $\mathfrak{D}_{12}^{\bullet}, \mathfrak{D}_{11^{\prime}}^{\bullet}, \mathfrak{D}_{1^{\prime} 2}^{\bullet}$, then $\mathfrak{H}^{\bullet}=\mathfrak{D}_{12}^{\bullet} \otimes \mathfrak{D}_{11^{\prime}}^{\bullet} \otimes \mathfrak{D}_{1^{\prime} 2}^{\bullet}$. Since the canonical line bundle on an affine space carries a canonical flat connection, the algebras $\mathfrak{D}_{i j}^{\bullet}$ admit $\left(\mathrm{GL}\left(V_{i}\right) \times \mathrm{GL}\left(V_{j}\right)\right)$-equivariant anti-involutions. Thus we can identify $\mathfrak{H}^{\bullet} \stackrel{\sim}{\longrightarrow} \mathfrak{D}_{12}^{\bullet} \otimes\left(\mathfrak{D}_{11^{\prime}}^{\bullet} \otimes \mathfrak{D}_{1^{\prime} 2}^{\bullet}\right)^{\text {op }}$.

The algebra $\mathfrak{H}^{\bullet}$ has a cyclic holonomic left dg-module $\mathfrak{Q}^{\bullet}$ of "delta-functions along the subvariety cut out by the equation $A=A^{\prime} A^{\prime \prime \prime}$, see (3.4.1). We also consider the $\mathrm{GL}\left(V_{1}\right) \times \mathrm{GL}\left(V_{1}^{\prime}\right)$-equivariant cyclic $\mathfrak{D}_{11^{\prime}}^{\bullet}$-module $\mathfrak{D}_{11^{\prime} 0}^{\bullet}$ corresponding

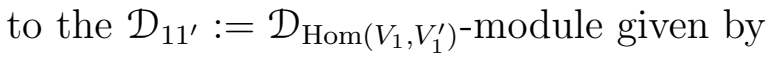

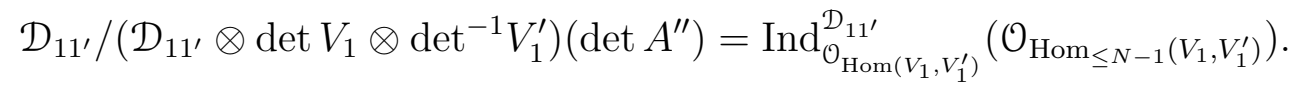

We define the following endofunctors of $D_{\text {perf }}^{G_{\overline{0}}}\left(\mathfrak{D}_{1,1}^{\bullet}\right)$ :

$$
\begin{aligned}
& D_{\text {perf }}^{G_{\overline{0}}}\left(\mathfrak{D}_{1,1}^{\bullet}\right)=D_{\text {perf }}^{\mathrm{GL}\left(V_{1}^{\prime}\right) \times \mathrm{GL}\left(V_{2}\right)}\left(\mathfrak{D}_{1^{\prime} 2}^{\bullet}\right) \ni M \mapsto p_{*} q^{*} M \\
& :=\left(\mathfrak{Q}^{\bullet} \otimes_{\mathfrak{D}_{11^{\prime}}, \otimes \mathfrak{D}_{1^{\prime} 2}^{\bullet}}\left(\mathfrak{D}_{11^{\prime}}^{\bullet} \otimes M\right)\right)^{\mathrm{GL}\left(V_{1}^{\prime}\right)} \in D_{\text {perf }}^{\mathrm{GL}\left(V_{1}\right) \times \mathrm{GL}\left(V_{2}\right)}\left(\mathfrak{D}_{12}^{\bullet}\right)=D_{\text {perf }}^{G_{\overline{0}}}\left(\mathfrak{D}_{1,1}^{\bullet}\right) \\
& D_{\text {perf }}^{G_{\overline{0}}}\left(\mathfrak{D}_{1,1}^{\bullet}\right)=D_{\text {perf }}^{\mathrm{GL}\left(V_{1}^{\prime}\right) \times \mathrm{GL}\left(V_{2}\right)}\left(\mathfrak{D}_{1^{\prime} 2}^{\bullet}\right) \ni M \mapsto p_{0 *} q_{0}^{*} M \\
& :=\left(\mathfrak{Q}^{\bullet} \otimes_{\mathfrak{D}_{1^{\prime}}, \otimes \mathfrak{D}_{1^{\prime} 2}^{\bullet}}\left(\mathfrak{D}_{11^{\prime} 0}^{\bullet} \otimes M\right)\right)^{\mathrm{GL}\left(V_{1}^{\prime}\right)} \in D_{\text {perf }}^{\mathrm{GL}\left(V_{1}\right) \times \mathrm{GL}\left(V_{2}\right)}\left(\mathfrak{D}_{12}^{\bullet}\right)=D_{\text {perf }}^{G_{\overline{0}}}\left(\mathfrak{D}_{1,1}^{\bullet}\right)
\end{aligned}
$$

Then similarly to Proposition 3.12.1 one proves

Proposition 5.3.1. (a) There is an isomorphism of functors

$$
\bar{\jmath}_{0 *} \bar{\jmath}_{0} \circ \Phi_{\hbar}^{1,1} \simeq \Phi_{\hbar}^{1,1} \circ p_{*} q^{*}: D_{\text {perf }}^{G_{\overline{0}}}\left(\mathfrak{D}_{1,1}^{\bullet}\right) \rightarrow D_{! * \mathbf{G}_{\mathbf{O}} \rtimes \mathbb{C} \times}(\mathbf{G r} \times \mathbf{V}) .
$$

(b) There is an isomorphism of functors

$$
\jmath_{0 *} \jmath_{0}^{!} \circ \Phi_{\hbar}^{1,1} \simeq \Phi_{\hbar}^{1,1} \circ p_{0 *} q_{0}^{*}: D_{\text {perf }}^{G_{\overline{0}}}\left(\mathfrak{D}_{1,1}^{\bullet}\right) \rightarrow D_{! * \mathbf{G}_{\mathbf{O}} \rtimes \mathbb{C} \times}(\mathbf{G r} \times \mathbf{V}) .
$$


Now using Proposition 5.3.1 in place of Proposition 3.12.1, one checks the monoidal properties of $\Phi_{\hbar}^{2,0}$ and $\Phi_{\hbar}^{0,2}$ similarly to the monoidal properties of $\Phi^{2,0}$ and $\Phi^{0,2}$ checked in Sections 3.14 and 3.15 respectively. This completes the proof of Theorem 5.1.1.

5.4. $\mathfrak{D}$-modules and $D_{\mathrm{GL}(N-1, \mathbf{O}) \rtimes \mathbb{C} \times}(\mathbf{G r})$. Similarly to Section 5.1 we consider the dg-algebra (with trivial differential) $\overline{\mathfrak{D}} \bullet$ of "graded differential operators" on $\operatorname{Hom}\left(V_{2}, \bar{V}_{1}\right)$. Then, similarly to Theorem 4.1.1, one proves

Theorem 5.4.1. There exists an equivalence of triangulated categories $\bar{\Phi}_{\hbar}: D_{\text {perf }}^{\bar{G}_{\overline{0}}}\left(\overline{\mathfrak{D}}^{\bullet}\right) \stackrel{\sim}{\longrightarrow} D_{\overline{\mathbf{G}}_{\mathbf{O}} \rtimes \mathbb{C}^{\times}}(\mathbf{G r})$ commuting with the left convolution action of the monoidal spherical Hecke category $\operatorname{Perv}_{\mathrm{GL}(N-1, \mathbf{O}) \rtimes \mathbb{C}^{\times}}\left(\mathbf{G r}_{N-1}\right) \cong \operatorname{Rep}\left(\mathrm{GL}_{N-1}\right)$ and with the right convolution action of the monoidal spherical Hecke category $\operatorname{Perv}_{\mathrm{GL}(N, \mathbf{O}) \rtimes \mathbb{C}^{\times}}\left(\mathbf{G r}_{N}\right) \cong \operatorname{Rep}\left(\mathrm{GL}_{N}\right)$.

\section{REFERENCES}

[AG1] S. Arkhipov, D. Gaitsgory, Differential operators on the loop group via chiral algebras, Int. Math. Res. Not. (2002), no. 4, 165-210.

[AG2] S. Arkhipov, D. Gaitsgory, Another realization of the category of modules over the small quantum group, Adv. Math. 173 (2003), no. 1, 114-143.

[B] R. Bezrukavnikov, On two geometric realizations of an affine Hecke algebra, Publ. Math. Inst. Hautes Études Sci. 123 (2016), 1-67.

[BD] A. Beilinson, V. Drinfeld, Quantization of Hitchin's integrable system and Hecke eigensheaves, https://math.uchicago.edu/ ${ }^{\sim}$ rinfeld/langlands/ QuantizationHitchin.pdf

[BF] R. Bezrukavnikov, M. Finkelberg, Equivariant Satake category and KostantWhittaker reduction, Moscow Math. Journal 8 (2008), no. 1, 39-72.

[Bra] T. Braden, Hyperbolic localization of intersection cohomology, Transform. Groups 8 (2003), 209-216.

$[\mathrm{BrF}] \quad$ A. Braverman, M. Finkelberg, A quasi-coherent description of the category $D-\bmod \left(\mathbf{G r}_{\mathrm{GL}(n)}\right)$, arXiv:1809.10774.

[C] J. Cogdell, Lectures on L-functions, converse theorems, and functoriality for $\mathrm{GL}_{n}$, Fields Inst. Monogr. 20, Amer. Math. Soc., Providence, RI (2004), 1-96.

[DG] V. Drinfeld, D. Gaitsgory, On a theorem of Braden, Transform. Groups 19 (2014), 313-358.

[FGT] M. Finkelberg, V. Ginzburg, R. Travkin, Mirabolic affine Grassmannian and character sheaves, Selecta Math. (N.S.) 14 (2009), no. 3-4, 607-628.

[FI] M. Finkelberg, A. Ionov, Kostka-Shoji polynomials and Lusztig's convolution diagram, Bull. Inst. Math. Acad. Sin. (N.S.) 13 (2018), no. 1, 31-42.

[GW] D. Gaiotto, E. Witten, $S$-duality of boundary conditions in $\mathcal{N}=4$ super Yang-Mills theory, Adv. Theor. Math. Phys. 13 (2009), no. 3, 721-896.

[G1] D. Gaitsgory, Twisted Whittaker model and factorizable sheaves, Selecta Math. (N.S.) 13 (2008), no. 4, 617-659.

[G2] D. Gaitsgory, Sheaves of categories and the notion of 1-affineness, Contemp. Math. 643 (2015), 127-225.

[G3] D. Gaitsgory, Quantum Langlands Correspondence, arXiv:1601.05279. 
[G4] D. Gaitsgory, The local and global versions of the Whittaker category, Pure Appl. Math. Q. 16 (2020), no. 3, 775-904.

[JPS] H. Jacquet, I. Piatetski-Shapiro, J. Shalika, Rankin-Selberg convolutions, Amer. J. Math. 105 (1983), no. 2, 367-464.

[LP] L. Le Bruyn, C. Procesi, Semisimple representations of quivers, Trans. Amer. Math. Soc. 317 (1990), no. 2, 585-598.

[L] G. Lusztig, Green polynomials and singularities of unipotent classes, Adv. in Math. 42 (1981), no. 2, 169-178.

[M] S. Mac Lane, Categories for the Working Mathematician, Graduate Texts in Mathematics 5 (2nd ed.) New York, Springer-Verlag (1998).

[MR] I. Mirković, S. Riche, Linear Koszul duality, Compositio Math. 146 (2010), no. 1, 233-258.

[MV] I. Mirković, K. Vilonen, Geometric Langlands duality and representations of algebraic groups over commutative rings, Ann. of Math. (2) 166 (2007), no. 1, 95-143. Erratum, Ann. of Math. (2) 188 (2018), no. 3, 1017-1018.

[MW] V. Mikhaylov and E. Witten, Branes and Supergroups, Comm. Math. Phys. 340 (2015), no. 2, 699-832.

[Sa] Y. Sakellaridis, Spherical functions on spherical varieties, Amer. J. Math. 135 (2013), no. 5, 1291-1381.

[Sh] T. Shoji, Green functions attached to limit symbols, Adv. Stud. Pure Math. 40 (2004), 443-467.

[Sch] S. Schieder, Monodromy and Vinberg fusion for the principal degeneration of the space of G-bundles, Ann. Sci. Éc. Norm. Supér. (4) 52 (2019), no. 4, 821-866.

[T] R. Travkin, Mirabolic Robinson-Shensted-Knuth correspondence, Selecta Math. (N.S.) 14 (2009), no. 3-4, 727-758.

[Z1] Y. Zhao, Quantum parameters of the geometric Langlands theory, arXiv: 1708.05108

[Z2] Y. Zhao, Notes on quantum parameters (GL-2), https://lysenko.perso.math.cnrs.fr/Notes_talks_winter2018/GL-2\%28Yifei\%29.pdf

Department of Mathematics, University of Toronto and Perimeter Institute of Theoretical Physics, Waterloo, Ontario, Canada, N2L 2Y5;

Skolkovo Institute of Science And Technology

Email address: braval@math.toronto.edu

National Research University Higher School of Economics, Russian Federation, Department of Mathematics, 6 Usacheva st, 119048 Moscow;

Skolkovo Institute of Science and Technology;

Institute for the Information Transmission Problems

Email address: fnklberg@gmail.com

Department of Mathematics, University of Chicago, Chicago, USA

Email address: vityaginzburg@gmail.com

Skolkovo Institute of Science and Technology, Moscow, Russia

Email address: roman.travkin2012@gmail.com 